\title{
SEARCH FOR HADRONIC RESONANCES IN MULTIJET FINAL STATES WITH THE CDF DETECTOR
}

BY CLAUDIA SEITZ

\author{
A thesis submitted to the \\ Graduate School-New Brunswick \\ Rutgers, The State University of New Jersey \\ in partial fulfillment of the requirements \\ for the degree of \\ Master of Science \\ Graduate Program in Physics and Astronomy \\ Written under the direction of \\ Prof. Eva Halkiadakis \\ and approved by
}

New Brunswick, New Jersey

January, 2011 


\title{
ABSTRACT OF THE THESIS
}

\section{Search for Hadronic Resonances in Multijet Final States with the CDF Detector}

\author{
by Claudia Seitz \\ Thesis Director: Prof. Eva Halkiadakis
}

This thesis describes a search for a new hadronic resonance in $3.2 \mathrm{fb}^{-1}$ of data using the Collider Detector at Fermilab. The Fermilab Tevatron accelerator collides beams of protons and antiprotons at a center of mass energy of $\sqrt{s}=1.96 \mathrm{TeV}$. A unique approach is presented to extract multijet resonances from the large QCD background. Although the search is model independent, a pair produced supersymmetric gluino decaying through R-parity violation into three partons each is used to test our sensitivity to new physics. We measure these partons as jets, and require a minimum of six jets in an event. We make use of the kinematic features and correlations and use an ensemble of jet combinations to distinguish signal from multijet QCD backgrounds. Our background estimates also include all-hadronic $t \bar{t}$ decays that have a signature similar to signal. We observe no significant excess in an invariant mass range of $77 \mathrm{GeV} / c^{2}$ to $240 \mathrm{GeV} / c^{2}$ and place $95 \%$ C.L. limits on $\sigma(p \bar{p} \rightarrow \tilde{g} \tilde{g} \rightarrow 3 j e t s+3 j e t s)$ as a function of gluino invariant mass. 


\section{Acknowledgements}

I want to begin by thanking my advisor Prof. Eva Halkiadakis as well as Prof. Amit Lath, whom without this thesis would have not been possible. I am grateful for all the help that allowed me to accomplish this project. Their enthusiasm and dedication for their work served as an inspiration. Furthermore I want to thank Prof. Scott Thomas who always has an open ear for theory questions as well as Tim Lou, Gautam Jain and Daryl Hare who laid a great foundation for this project. Many thanks to my office mates A.J. Richards and Shruti Panwalker who were great company in the early beginning of me joining the High Energy group here at Rutgers and also for great conversations during lunch breaks. However, my stay in the US would have never been possible if it were not for the "Amerika-Programm" and the Baron-von-Swaine scholarship from the University of Würzburg, Germany.

In addition to the new academic experiences that I had here at Rutgers, I am also grateful for the opportunity to live in a new country, learn a new language and meet people from all over the world. Vielen Dank an Sabine Keiber, die mir zeigte neben harter Arbeit auch die schönen Seiten des Lebens zu genießen.

I want to thank my friends Mike, Eliav, Sebastian and many more who made my life here so enjoyable. My special thanks belong to Christian, who stayed with me the long nights in the lab and supported me throughout this project and will hopefully be part of my life for years to come. Thanks to Megan and Emmanuel who offered me a family while my own was far away. I am thankful to be able to live in an era where the internet is easily accessible for almost everyone. This made it possible for me to stay in contact with my friends, like Sascha, Evi, Lisa and Fine.

Außerdem möchte ich meinen Eltern, Jürgen und Martina, wie auch meinen Großeltern, Rainer und Helga danken. Ich hoffe ihr seit stolz auf mich. Danke. 


\section{Dedication}

To life and the decisions we make. 


\section{Table of Contents}

Abstract .................................. ii

Acknowledgements ........................ ii

Dedication .............................. iv

List of Tables . . . . . . . . . . . . . . . . . . . . . . viii

List of Figures $\ldots \ldots \ldots \ldots \ldots \ldots \ldots \ldots \ldots$ ix

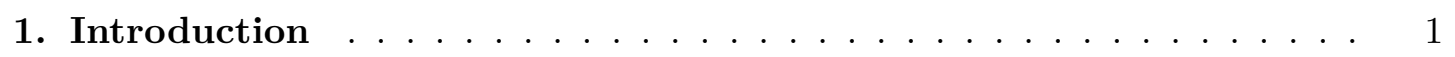

2. Theoretical overview . . . . . . . . . . . . . . . 3

2.1. The Standard Model . . . . . . . . . . . . . . . . . . . . 3

2.1.1. Elementary Fermions . . . . . . . . . . . . . . . . . 3

2.1.2. Elementary Bosons . . . . . . . . . . . . . . . 5

2.1.3. Field theoretical description . . . . . . . . . . . . 5

2.1.4. Higgs Mechanism . . . . . . . . . . . . . . . 6

2.2. Quantum Chromo Dynamics . . . . . . . . . . . . 7

2.2.1. Quark Confinement ................. 7

2.2.2. Hadronization . . . . . . . . . . . . . . . . 9

2.3. Issues with the Standard Model and SUSY . . . . . . . . . . . . . . 10

2.3.1. Minimal Supersymmetric Standard Model . . . . . . . . . . . . . 12

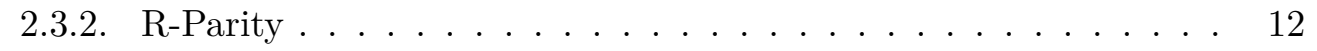

2.3.3. Particles in the MSSM . . . . . . . . . . . . . 13

2.3.4. R-parity violation . . . . . . . . . . . . . . . 14

2.4. Multijet Signals $\ldots \ldots \ldots \ldots$

2.4.1. Top Quark . . . . . . . . . . . . . . . . 15 


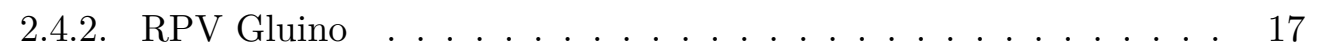

3. The experimental apparatus $\ldots \ldots \ldots \ldots \ldots \ldots$

3.1. The Tevatron Complex. . . . . . . . . . . . . . . . . . . . 18

3.1.1. Proton source . . . . . . . . . . . . . . . . . . . . . 18

3.1.2. Main injector . . . . . . . . . . . . . . . . . . . . . . . 19

3.1.3. The Antiproton source . . . . . . . . . . . . . . . . . 19

3.1.4. The Tevatron . . . . . . . . . . . . . . . . . . . . . . 20

3.2. CDF - Collider Detector at Fermilab . . . . . . . . . . . . . . 21

3.2.1. Detector Coordinates . . . . . . . . . . . . . . . 22

3.2 .2 . Tracking System . . . . . . . . . . . . . . . . . 23

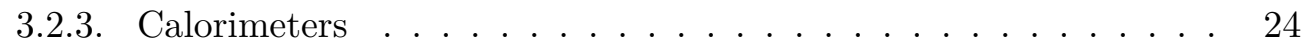

3.2.4. Muon detectors . . . . . . . . . . . . . . . . . 26

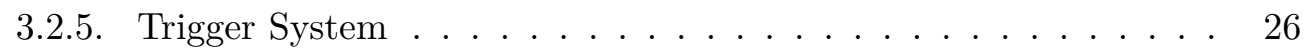

3.3. Datasets . . . . . . . . . . . . . . . . . . 27

3.4. Monte Carlo. . . . . . . . . . . . . . . . . . . . . . 27

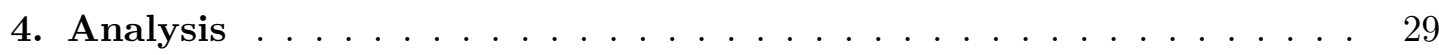

4.1. Event Reconstruction . . . . . . . . . . . . . . . . . . . . . 29

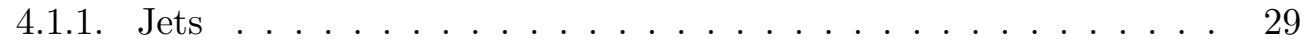

JetClu Algorithm at CDF . . . . . . . . . . . . . . . . 30

Jet energy corrections . . . . . . . . . . . . . . . . . 31

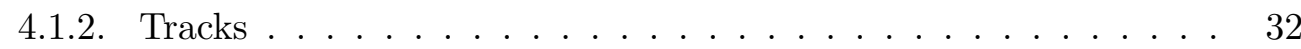

$4.1 .3 . \quad$ Primary Vertex . . . . . . . . . . . . . . . . . 33

4.1.4. Missing Transverse Energy _ . . . . . . . . . . . . . . 33

4.2. Event Selection . . . . . . . . . . . . . . . . . . . . . 34

4.2.1. Analysis Strategy . . . . . . . . . . . . . . . . . 34

4.2.2. Basic Selection . . . . . . . . . . . . . . . 35

4.2.3. Advanced Selection . . . . . . . . . . . . . . . . . . . 40

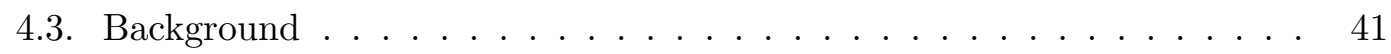


4.3.1. Data-driven QCD Background estimate . . . . . . . . . . . . 42

4.3.2. All-hadronic $t \bar{t}$ decay . . . . . . . . . . . . . . . . . . 43

4.4. Diagonal Cut Optimization . . . . . . . . . . . . . . . . . . . 45

4.4.1. Pseudoexperiments . . . . . . . . . . . . . . . . . 45

$4.4 .2 . \quad$ Optimization $\ldots \ldots \ldots \ldots \ldots$. . . . . . . . . . . . . . 48

4.5. Acceptance $\ldots \ldots \ldots \ldots \ldots$. . . . . . . . . . . . . . . . . 50

5. Setting Limits on Hadronic Resonances . . . . . . . . . . . . . . . . . 54

5.1. Bayesian approach for observed $95 \%$ confidence limit calculation . . . . 54

5.2. Expected $95 \%$ confidence limit . . . . . . . . . . . . . . . . . 55

5.3. Systematic uncertainties . . . . . . . . . . . . . . . . 56

$5.3 .1 . \quad$ Linearity check . . . . . . . . . . . . . . . 56 56

$5.3 .2 . \quad$ Background shape systematics . . . . . . . . . . 58

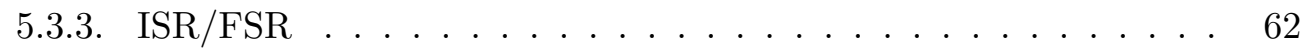

$5.3 .4 . \quad$ Jet Energy Scale . . . . . . . . . . . . . . . . . . . 62

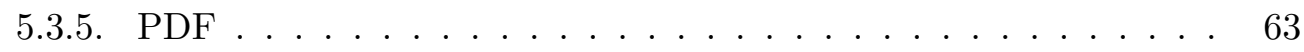

$5.3 .6 . \quad$ Luminosity . . . . . . . . . . . . . . . . . 63

5.3.7. Summary of systematics . . . . . . . . . . . . 64

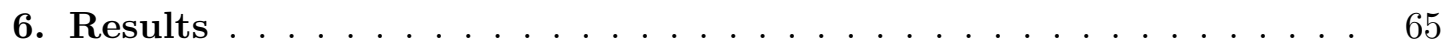

Appendix A. Studies for the Top quark . . . . . . . . . . . . 68

A.1. Different Monte Carlo Samples . . . . . . . . . . . . . . . . . . . 68

A.2. Dalitz plots . . . . . . . . . . . . . . . . 68

A.3. Identifying the b quark: b-tagging . . . . . . . . . . . . . . 70

A.3.1. W boson . . . . . . . . . . . . . . . . 70

A.4. Semi-leptonic decay . . . . . . . . . . . . . . . 73

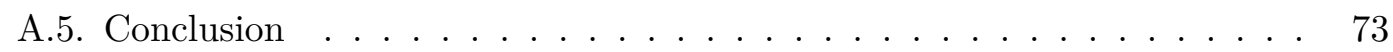




\section{List of Tables}

2.1. Particles and their quantum numbers in the Standard Model . . . . . . 4

2.2. Color and anti-color charge combinations for gluons . . . . . . . . . . . 7

2.3. Chiral supermultiplets in the Minimal Supersymmetric Standard Model 12

3.1. Signal MC gluino samples . . . . . . . . . . . . . . . . . . . . . . . 28

4.1. Quality flag for primary vertex . . . . . . . . . . . . . . . 33

4.2. Optimal diagonal cut for different gluino masses . . . . . . . . . . . 50

4.3. Gluino acceptance . . . . . . . . . . . . . . . . . . . . 50

5.1. Number of observed events at $95 \%$ C.L. . . . . . . . . . . . . . . 57

5.2. Effect of the jet energy scale on gluino acceptance . . . . . . . . . 63

6.1. Expected $95 \%$ C.L. limit and observed 95\% C.L. limit . . . . . . . . . 67

A.1. Expected top events from different MC samples . . . . . . . . . . . . . . 69 


\section{List of Figures}

2.1. Standard Model of Particle Physics . . . . . . . . . . . . . . . . . . . 5

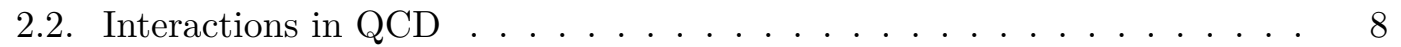

2.3. Hadronization Model . . . . . . . . . . . . . . . . . . . . . . 9

2.4. Cluster hadronization model . . . . . . . . . . . . . . . . . . . . . . . . . 10

2.5. String hadronization model . . . . . . . . . . . . . . . . . 11

2.6. $t \bar{t}$ decay in the all-hadronic channel resulting in at least 6 jets . . . . . . 16

2.7. Pair produced gluinos decaying into three jets each . . . . . . . . . . 17

3.1. Tevatron Accelerator complex at Fermilab . . . . . . . . . . . . . . . . . 19

3.2. Elevation view of half of the CDF Run II detector . . . . . . . . . . . 21

3.3. CDF Tracking System . . . . . . . . . . . . . . . . . . 23

4.1. $M_{j j j}$ versus $\sum_{j j j}\left|p_{T}\right| \ldots \ldots \ldots \ldots \ldots \ldots \ldots \ldots \ldots$

4.2. $\quad M_{j j j}$ versus $\sum_{j j j}\left|p_{T}\right|$ multiple entry scatter plots $\ldots \ldots \ldots \ldots$

4.3. Mass plots for gluino signal Monte Carlo . . . . . . . . . . . . . . . 37

4.4. $p_{T}$ distribution for the top 8 jets in the data and PYTHIA $t \bar{t}$ Monte Carlo 38

4.5. Distributions of missing $E_{T}$, number of vertices, number of jets, and the maximum $\left|z_{0}\right|$ of all jets in data and PYTHIA $t \bar{t}$ Monte Carlo before cuts. 39

4.6. $\quad \sum_{6 j e t} p_{T}$ distribution in data and PYTHIA $t \bar{t}$ Monte Carlo before and after basic and advanced selection and a diagonal cut of $100 \mathrm{GeV} / \mathrm{c})$. . . 39

4.7. $z$ rms distribution in data and PYTHIA $t \bar{t}$ Monte Carlo before the $z_{\text {rms }}$

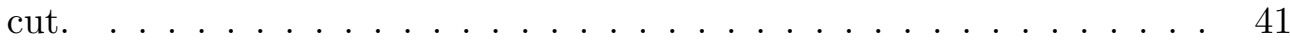

4.8. $\quad \sum_{j j j}\left|p_{T}\right|$ distributions for 5 -jet and 6 -jet sample $\ldots \ldots \ldots \ldots$

4.9 .5 -jet and 6 -jet background . . . . . . . . . . . . . . . 45

4.10. Landau parameters for 5 -jet and 6 -jet background . . . . . . . . . . 46

4.11.6-jet background shape . . . . . . . . . . . . . . . . . . . . . 47 
4.12. Diagonal cut optimization for for a gluino mass of $110 \mathrm{GeV} / c^{2}$. . . . 49

4.13. Gluino signal/background fraction as a function of diagonal cut . . . . . 51

4.14. Optimal diagonal cut for each gluino mass. . . . . . . . . . . . . . 52

4.15. Acceptance for each gluino mass from $74 \mathrm{GeV} / c^{2}$ to $245 \mathrm{GeV} / c^{2}$. We note that the acceptance is independent of the mass. . . . . . . . . . . 52

5.1. Example for calculation of $95 \%$ confidence limit . . . . . . . . . . . . 55

5.2. Measured cross section distribution for 1000 pseudoexperiments at a mass of $158 \mathrm{GeV} / c^{2}$ without systematic uncertainties. . . . . . . . . . 56

5.3. Input cross section vs. measured cross section for varying masses. . . . . 58

5.4. Cross section limit plot with background systematic (1) . . . . . . . . . 59

5.5. Landau width with symmetric errors around the mean of 5jet and 6jet. 61

5.6. Landau mean with symmetric errors around the mean of 5jet and 6jet. 61

5.7. Cross section limit plot with with overall shift of background parameters

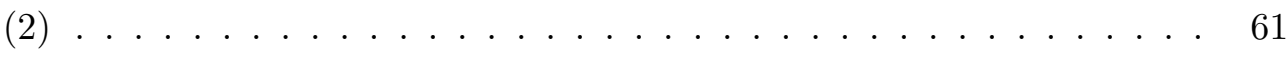

5.8. Comparison of more/less ISR/FSR . . . . . . . . . . . . . . . . 62

5.9. Cross section limit plot showing effect of systematics . . . . . . . . . 64

6.1. $95 \%$ C.L. cross section limit plot . . . . . . . . . . . . . . 66

A.1. The three combination of Dalitz variables in $t \bar{t}$ Monte Carlo inside the mass window. Left: high vs low, Middle: high vs mid, Right: mid vs low 71

A.2. Dalitz plots $t \bar{t} \mathrm{MC} \ldots \ldots \ldots \ldots \ldots \ldots \ldots$

A.3. Dalitz plots data . . . . . . . . . . . . . . . . 71

A.4. Dalitz variables . . . . . . . . . . . . . . . . . . . 72

A.5. Anti b-tagged dijet mass . . . . . . . . . . . . . . . 72 


\section{Chapter 1}

\section{Introduction}

What is the world made of, where does mass come from? These and similar questions have been fascinating humans for thousands of years. Piece by piece we developed natural science to investigate these and many other questions. Today we live in a century full of technological advancements that people two hundred years ago could not have even imagined. Still there are many unsolved questions. With the invention of the atomic model in the early 20th century we began to probe deeper into the fundamental constituents of our universe. We saw phenomena that were not explainable by means of classical theory. Quantum mechanics was born and only shortly after quantum field theory came to life. Since then people have developed this theory that lead to the most successful and precise model in physics, the Standard Model of particle physics. Everything started with the antiparticle of the electron, the positron. Soon after new particles were predicted by the theory and observed by experiments as well as new phenomena were observed and included into the theory. The last big discovery happened in 1995 at the Tevatron collider at Fermilab, when the top quark was found. Since then many efforts have been made in theory and experiment to find new physics beyond the Standard Model. Another crucial problem is the source of spontaneous symmetry breaking which has not yet been resolved. There are many theories concerning this issue and the most accepted one seems to be the so-called Higgs mechanism which also predicts the existence of a Higgs boson. However, so far there is no experimental evidence for this particle.

This thesis describes a search for new physics beyond the Standard Model. We will focus mainly on multijet physics, which for a long time was an almost neglected aspect of the Standard Model. The underlying theory of Quantum Chromo Dynamics 
is the least understood part of the Standard Model but new physics might be hidden behind strong couplings. Chapter 2 focuses on the theory behind this search. We are using data produced by the Tevatron hadron collider at a center of mass energy of $\sqrt{s}=1.96 \mathrm{TeV}$ and collected by the Collider Detector at Fermilab (CDF). Chapter 3 describes the experimental setup. The backgrounds are large for multijet processes at hadron colliders. We will present a unique approach to extract a 3-jet resonance. This analysis technique is discussed in Chapter 4. Even though the presented search is model independent we use a R-parity violating gluino process to set limits on its cross section which is described in Chapter 5. Ideas to improve this technique and for further investigation of possible new physics signals are briefly discussed in Appendix A. 


\section{Chapter 2}

\section{Theoretical overview}

\subsection{The Standard Model}

The Standard Model of particle physics can be considered one of the most successful theories developed in the last century. It is a relativistic invariant quantum field theory and describes the electromagnetic (QED), weak and strong (QCD) interactions between elementary particles. The fourth fundamental force, gravity, is not included into the Standard Model since the effects are negligible in the energy region we are able to access with current experiments. By means of group theory it can be described as:

$$
S U(3) \times S U(2) \times U(1)
$$

where $\mathrm{SU}(3)$ is an abstract representation for the strong force and $S U(2) \times U(1)$ describes the electroweak force. So far this theory has predicted experimental results with an extremely high precision. However, there are still unanswered questions such as how particles acquire mass. Another aspect of high energy physics is to find new physics beyond the Standard Model. There are many different models which predict new physics processes and one of them shall be discussed in Section. 2.3. Table 2.1 and Figure 2.1 show the the constituents of the Standard Model.

\subsubsection{Elementary Fermions}

Elementary particles that build up all the visible matter in the universe can be divided into two major categories, fermions and bosons. The most fundamental difference between the two types is their spin. Fermions have half-integer spin and are for example the constituents of protons and neutrons. Bosons on the other hand have integer spin 
and act as mediators of the three fundamental forces: electromagnetic, weak, and strong.

\begin{tabular}{cccccc} 
Name & Spin & $\begin{array}{c}\text { Baryon } \\
\text { Number B }\end{array}$ & $\begin{array}{c}\text { Lepton } \\
\text { Number L }\end{array}$ & $\begin{array}{c}\text { Charge } \\
\text { Q }\end{array}$ & $\begin{array}{c}\text { Mass } \\
{[\mathrm{MeV}]}\end{array}$ \\
\hline leptons & & & & & \\
\hline electron & $\frac{1}{2}$ & 0 & 1 & -1 & 0.511 \\
electron neutrino & $\frac{1}{2}$ & 0 & 1 & 0 & $<2.2 \cdot 10^{-6}$ \\
muon & $\frac{1}{2}$ & 0 & 1 & -1 & 105.7 \\
muon neutrino & $\frac{1}{2}$ & 0 & 1 & 0 & $<0.17$ \\
tau & $\frac{1}{2}$ & 0 & 1 & -1 & $1.77 \cdot 10^{3}$ \\
tau neutrino & $\frac{1}{2}$ & 0 & 1 & 0 & $<15.5$ \\
\hline quarks & & & & & \\
\hline up & $\frac{1}{2}$ & $\frac{1}{3}$ & 1 & $\frac{2}{3}$ & 2.4 \\
down & $\frac{1}{2}$ & $\frac{1}{3}$ & 1 & $-\frac{1}{3}$ & 4.8 \\
charm & $\frac{1}{2}$ & $\frac{1}{3}$ & 1 & $\frac{2}{3}$ & $1.27 \cdot 10^{3}$ \\
strange & $\frac{1}{2}$ & $\frac{1}{3}$ & 1 & $-\frac{1}{3}$ & 104 \\
top & $\frac{1}{2}$ & $\frac{1}{3}$ & 1 & $\frac{2}{3}$ & $171.2 \cdot 10^{3}$ \\
bottom & $\frac{1}{2}$ & $\frac{1}{3}$ & 1 & $-\frac{1}{3}$ & $4.2 \cdot 10^{3}$ \\
\hline gauge bosons & & & & & \\
\hline photon & 1 & 0 & 0 & 0 & 0 \\
W boson & 1 & 0 & 0 & \pm 1 & $80.4 \cdot 10^{3}$ \\
Z boson & 1 & 0 & 0 & 0 & $91.2 \cdot 10^{3}$ \\
gluon & 1 & 0 & 0 & 0 & 0
\end{tabular}

Table 2.1: Particles and their quantum numbers in the Standard Model Spin is defined in units of $\hbar$ and electrical charge in units of the elementary charge e. [1] and [2]

Leptons interact through the electroweak force, they are undisturbed by the strong force. With spin $\frac{1}{2}$, leptons belong to the family of fermions. Each lepton is assigned a Lepton number $\mathrm{L}=1$ and for each anti-lepton $\mathrm{L}=-1$. All observed decay processes show a conservation of Lepton number. The second part of the fermion family are quarks. The quark model was established by Murray Gell-Mann in the 1960s and describes that all observed matter is made of smaller pieces which he called quarks [3]. Quarks are assigned a Baryon number $\mathrm{B}=\frac{1}{3}$ and anti-quarks have $\mathrm{B}=-\frac{1}{3}$. Quarks can not be observed as a single particle and they always confine together to build baryons (3 quarks, $\mathrm{B}= \pm 1$ ) and mesons $(2$ quarks, $\mathrm{B}=0)$. Baryon number also seems to be a conserved quantity and there is no experimental evidence for its violation. However, the difference of Baryon and Lepton numbers, B-L, is always conserved. 


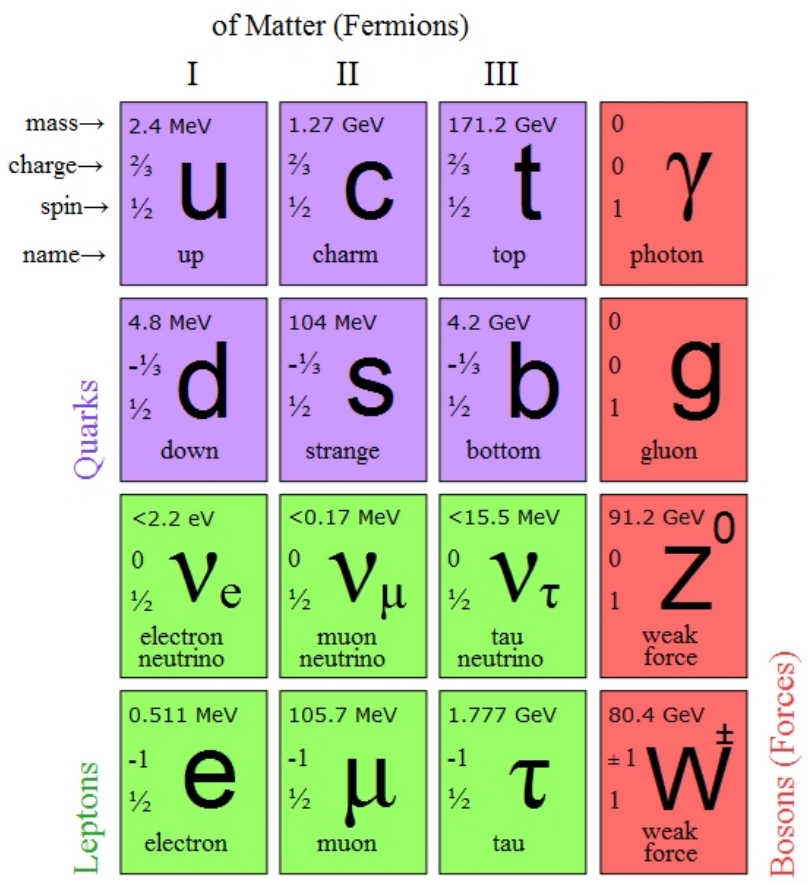

Figure 2.1: Standard Model of Particle Physics [2]

\subsubsection{Elementary Bosons}

The Standard Model has four different gauge bosons which act as mediators between the elementary particles. All of these bosons have spin 1. The photon, $\gamma$, is the massless mediator of the electromagnetic force. The weak force has three different massive representatives, the charged $W^{+}, W^{-}$as well as the neutral $Z^{0}$. The interaction between quarks, the strong color force, is mediated by massless gluons.

\subsubsection{Field theoretical description}

The electromagnetic and weak interaction can be combined by starting with the free particle Lagrangian:

$$
L_{0}=i \overline{\mathbf{\Psi}} \gamma^{\mu} \partial_{\mu} \boldsymbol{\Psi}
$$

where $\boldsymbol{\Psi}$ is the two dimensional Dirac Spinor. By replacing the covariant derivative $\partial_{\mu}$ with

$$
D_{\mu}=\partial_{\mu}+i g \mathbf{W}_{\mu} \cdot \mathbf{T}+i g^{\prime} \frac{1}{2} B_{\mu} Y
$$


and adding kinetic energy terms for the gauge fields $-\frac{1}{4} \mathbf{W}_{\mu \nu} \cdot \mathbf{W}^{\mu \nu}-\frac{1}{4} B_{\mu \nu} \cdot B^{\mu \nu}$ an invariance under the combined gauge transformation $S U(2) \times U(1)$ can be achieved. Equation 2.3 contains the two gauge fields $\mathbf{W}_{\mu}$ and $B_{\mu}$ which contain photon, $W^{ \pm}$and $Z^{0}$ bosons. $g$ and $g^{\prime}$ are coupling constants for electromagnetic and weak interaction. $\mathbf{T}$ is the the weak isospin operator that generates the $\mathrm{SU}(2)$ symmetry transformation and Y represents the weak hypercharge [4]. This model does not give the full information about the gauge bosons. We know experimentally that the carriers of the weak force are massive, however, adding mass terms to the Lagrangian in equation 2.2 would destroy the gauge invariance of the theory. Therefore, we need to find another mechanism to give gauge bosons mass, the so-called Higgs mechanism.

\subsubsection{Higgs Mechanism}

Adding a scalar field in the form of an isospin doublet $\mathbf{\Phi}=\left(\Phi_{1}, \Phi_{2}\right)$ to the Lagrangian leads to a spontaneous symmetry breaking which creates massive gauge bosons. The Higgs Lagrangian is described by equation 2.4 :

$$
L_{H}=\left|D_{\mu} \boldsymbol{\Phi}\right|^{2}-\mu^{2} \boldsymbol{\Phi}^{+} \boldsymbol{\Phi}+\lambda\left(\boldsymbol{\Phi}^{+} \boldsymbol{\Phi}\right)^{2}
$$

By choosing the parameters $\lambda$ and $\mu$ accordingly one can create a non-vanishing vacuum

expectation value (vev) of $\left|\Phi_{v e v}\right|=\sqrt{\frac{1}{2} \frac{\mu^{2}}{\lambda}}$ [4]. The former massless fermions will couple through a Yukawa coupling to the Higgs field and acquire mass in this process. The Higgs boson has not been observed yet but a lower mass limit at $95 \%$ C.L. of $114 \mathrm{GeV}$ was set by LEP (Large Electron Positron collider) at CERN [5]. The newest limits on the Higgs mass from July 2010 from the Tevatron accelerator at Fermilab near Chicago exclude a region of $158 \mathrm{GeV} / \mathrm{c}^{2}<m_{H}<175 \mathrm{GeV} / \mathrm{c}^{2}$ at a $95 \%$ C.L. [6]. 


\subsection{Quantum Chromo Dynamics}

In this analysis we are looking for new physics that involves strong coupling and therefore is described by Quantum Chromo Dynamics (QCD). Quarks and gluons are the main participants in this sector of the Standard Model. The description of QCD is mainly derived from QED. Instead of one type of electric charge the strong interaction is described by three different color charges red (r), green (g) and blue (b), carried by quarks, as well as their anti-colors $(\bar{r}, \bar{g}, \bar{b})$, carried by antiquarks. This leads to a more complex structure than in QED [7]. The underlying symmetry group $S U_{C}(3)$ builds a color octet and a color singlet, whereas only the color octet plays a role in QCD interactions. Therefore, there are eight gluons carrying combinations of colors and anti-colors shown in Table 2.2

$$
r \bar{b} \quad r \bar{g} \quad b \bar{g} \quad b \bar{r} \quad g \bar{r} \quad g \bar{b} \quad \frac{1}{\sqrt{2}}(r \bar{r}-b \bar{b}) \quad \frac{1}{\sqrt{6}}(r \bar{r}+b \bar{b}-2 g \bar{g})
$$

Table 2.2: Color and anti-color charge combinations for gluons [8].

The mentioned color singlet is the colorless combination $(\bar{r} r+\bar{g} g+\bar{b} b)$ which stays unchanged under rotation in the color space. This colorless state cannot function as a gluon carrying color charge between quarks [1]. Because of the charge properties of gluons they are able to interact among each other unlike photons in QED. Photons carry no charge by themselves and therefore cannot interact with each other. Figure 2.2 shows gluon-quark and gluon-gluon interactions.

\subsubsection{Quark Confinement}

The fact that quarks cannot be observed as single particles in nature is due to the socalled color confinement. Quarks undergo a hadronization process, described in more detail in Section 2.2.2, where they build mesons (quark, antiquark) or baryons (three quarks). To understand this process we need to look deeper into the nature of the strong interaction. From the electromagnetic interaction it is known that the electric potential decreases as $\approx \frac{1}{r}, \mathrm{r}$ being the radial distance from the electric charge. This leads to the fact that electrons for example can exist separately without being confined in a bound state. In QCD, however, the strong interaction can be described by an 

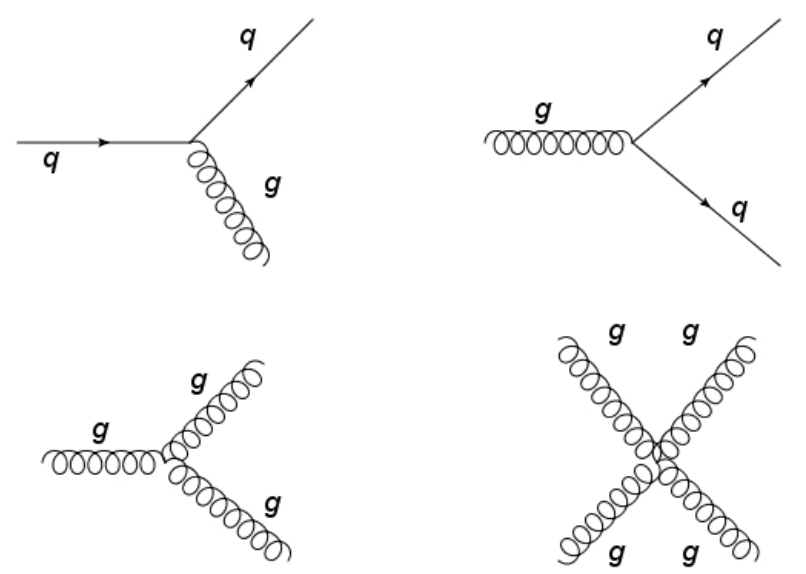

Figure 2.2: Interactions in QCD

Top: gluon emission, gluon absorption, Bottom: gluon-gluon interaction

empirical potential of the form [8]:

$$
V=-\frac{4}{3} \frac{\alpha_{s}}{r}+k r
$$

where $\alpha_{s}$ is the strong coupling constant and $\mathrm{r}$ describes the radial distance to the color charge. For short distances we assume a Coulomb like potential which is overlapped by a linear function that increases indefinitely at longer distances. The form of this potential was experimentally tested and seems to represent the interaction well. Even though we denote $\alpha_{s}$ as the coupling constant, it is actually not constant. $\alpha_{s}$ is a so-called running coupling constant which is a function of momentum transfer $\left(q^{2}\right)$ and the masses of the particles involved in the process. A discussion of running coupling constants in QCD and QED can be found in [1]. However, this strong coupling constant shows a somewhat special behavior. It decreases for high momentum transfers (large $q^{2}$ ) thus for small distance it can be described perturbatively in this regime. For small $q^{2}$ (thus for large distances) it increases indefinitely. 


\subsubsection{Hadronization}

As mentioned before, quarks cannot exist as single particles and therefore they undergo the process of hadronization. A full description of this process can be found in Reference [9]. Figure 2.3 shows the way of building hadrons after a hard collision. The produced partons undergo the so-called parton shower, which can be approximately calculated by means of perturbative QCD. After this point the distance between the

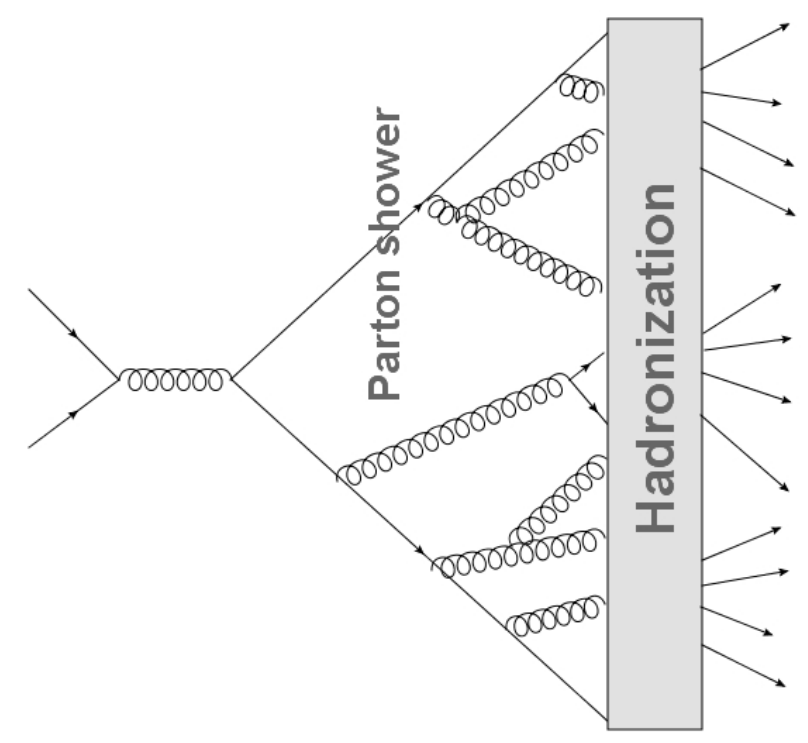

Figure 2.3: Hadronization Model

From the hard collision to final state hadrons.

partons increases (momentum transfer decreases) which leads to an increase in the coupling constant $\alpha_{s}$. This is a regime where the perturbative treatment starts to break down and a phenomenological model needs to be established. An illustration of this process can be seen in Figure 2.3. Two basic models are in use at the moment; the Cluster model and the String model (both follow the description in [10]). Figures 2.4 and 2.5 show illustrations for each model which are explained below.

- Cluster Model: After the parton shower, gluons are split into color-singlets $q \bar{q}$ which combine with other color-singlets into colorless clusters. These clusters 
decay afterwards into the known hadrons. This model has few parameters. It has a natural mechanism to generate transverse momenta and the production of heavy particles is suppressed. An illustration can be seen in Figure 2.4

- String Model: This model is described by a color flux between the initial colorsinglets $q \bar{q}$. When the partons move further away from each other the string gets stretched. Due to the strong color field it breaks up via quark-antiquark production until the energy in the string reaches a certain threshold. After that the remaining parts build the known hadrons. An illustration can be seen in Figure 2.5

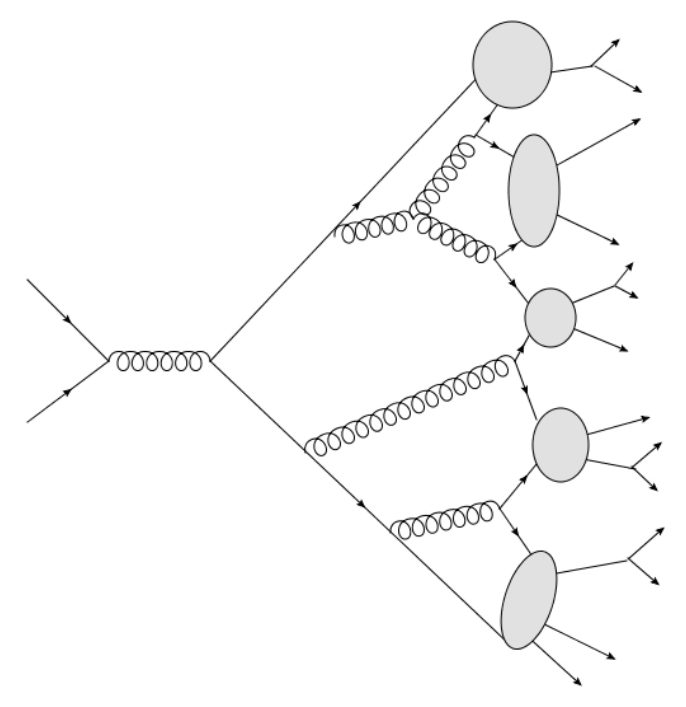

Figure 2.4: Cluster hadronization model

\subsection{Issues with the Standard Model and SUSY}

Even though the existing Standard Model gives precise predictions for experimental measurements there are still unsolved problems such as including gravity, understanding the mass hierarchy for fermions or explaining dark matter [11. Several different models have been developed and one of them, the Minimal Supersymmetric Standard Model (MSSM), is explained in this section. Supersymmetry (SUSY) imposes a fundamental 


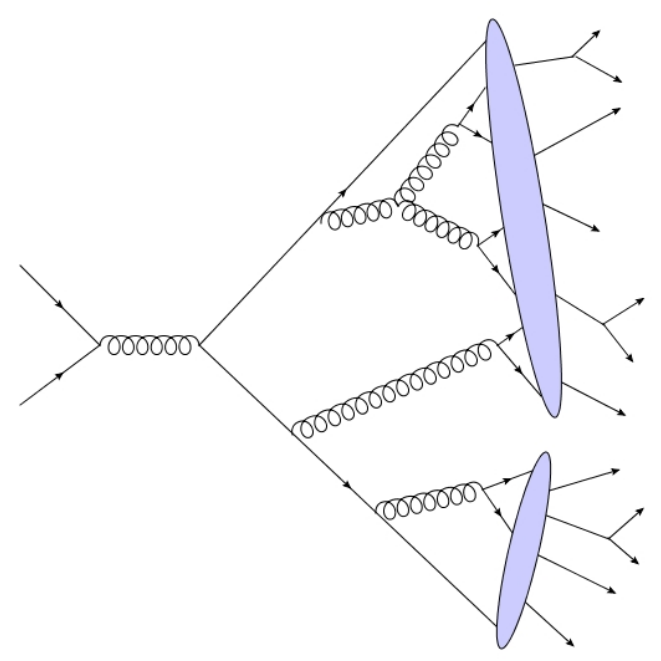

Figure 2.5: String hadronization model

symmetry between fermions and bosons. A supersymmetric operator Q can act on fermions as well as bosons.

$$
\mathrm{Q} \mid \text { fermion }>=\mid \text { boson }>\quad \mathrm{Q} \mid \text { boson }>=\mid \text { fermion }>\text {. }
$$

This implies that every Standard Model fermion (boson) has a corresponding bosonic (fermionic) superpartner. The standard notation is for bosonic superpartners to begin with the letter "s-" while fermionic superpartners end on the syllable "-ino". Supersymmetric particles are referred to as sparticles. The operator Q satisfies the algebra:

$$
\begin{gathered}
{\left[Q, Q^{\dagger}\right]=P^{\mu},} \\
{[Q, Q]=\left[Q^{\dagger}, Q^{\dagger}\right]=0,} \\
{\left[P^{\mu}, Q\right]=\left[P^{\mu}, Q^{\dagger}\right]=0,}
\end{gathered}
$$

where $P^{\mu}$ is the four-momentum generator. A single-particle state of a supersymmetric theory is called supermultiplet and consists of fermions and bosons. Particles in the same supermultiplet must have the same electric charge, weak isospin and color degree of freedom. This is due to the the fact the generators $\left[Q, Q^{\dagger}\right]$ commute with the generators of gauge transformations [12]. 


\subsubsection{Minimal Supersymmetric Standard Model}

There are many ways to introduce a supersymmetry into a theory, for example the Minimal Supersymmetric Standard Model (MSSM). The description in this section follows mainly Reference [12]. Since no sparticles have been observed, supersymmetry has to be a broken symmetry. It is desirable to start with a Lagrangian that preserves exact supersymmetry and introduce a spontaneous symmetry breaking . This is similar to the electroweak symmetry breaking introduced in the Standard Model.

The superpotential for a MSSM is given by:

$$
W_{M S S M}=\bar{u} \mathbf{y}_{\mathbf{u}} Q H_{u}-\bar{d} \mathbf{y}_{\mathbf{d}} Q H_{d}-\bar{e} \mathbf{y}_{\mathbf{e}} L H_{d}+\mu H_{u} H_{d}
$$

where $H_{u}, H_{d}, Q, L, \bar{u}, \bar{d}, \bar{e}$ are chiral supermultiplets which can be seen in Table 2.3 . $\mathbf{y}_{\mathbf{d}}, \mathbf{y}_{\mathbf{u}}, \mathbf{y}_{\mathbf{e}}$ are dimensionless Yukawa couplings in the form of $3 \times 3$ matrices. The last term in equation 2.9 represents the supersymmetric Higgs boson.

\begin{tabular}{ccccc} 
names & & spin 0 & spin $\frac{1}{2}$ & SU(3), $\mathbf{S U}(\mathbf{2}), \mathbf{U}(\mathbf{1})$ \\
\hline squarks, quarks & $\mathrm{Q}$ & $\left(\widetilde{u}_{L} \widetilde{d}_{L}\right)$ & $\left(u_{L} d_{L}\right)$ & $\left(3,2, \frac{1}{6}\right)$ \\
$(\times 3$ families $)$ & $\bar{u}$ & $\widetilde{u}_{R}^{*}$ & $u_{R}^{\dagger}$ & $\left(3,1,-\frac{2}{3}\right)$ \\
& $\bar{d}$ & $\widetilde{d}_{R}^{*}$ & $d_{R}^{\dagger}$ & $\left(\overline{3}, 1, \frac{1}{3}\right)$ \\
\hline sleptons, leptons & $\mathrm{L}$ & $\left(\widetilde{\nu} \widetilde{e}_{L}\right)$ & $\left(\nu e_{L}\right)$ & $\left(1,2,-\frac{1}{2}\right)$ \\
$(\times 3$ families $)$ & $\bar{e}$ & $\widetilde{e}_{R}^{*}$ & $e_{R}^{\dagger}$ & $(1,1,1)$ \\
\hline Higgs, higgsinos & $H_{u}$ & $\left(H_{u}^{+} H_{u}^{0}\right)$ & $\left(\widetilde{H}_{u}^{+} \widetilde{H}_{u}^{0}\right)$ & $\left(1,2,+\frac{1}{2}\right)$ \\
& $H_{d}$ & $\left(H_{d}^{0} H_{d}^{-}\right)$ & $\left(\widetilde{H}_{d}^{0} \widetilde{H}_{d}^{-}\right)$ & $\left(1,2,-\frac{1}{2}\right)$
\end{tabular}

Table 2.3: Chiral supermultiplets in the Minimal Supersymmetric Standard Model [12.

Further discussions of supersymmetric models can be found in References [13] and [12].

\subsubsection{R-Parity}

There are additional terms other than the ones shown in equation 2.9 that are gaugeinvariant but would violate Lepton and Baryon number conservation. These violations have not been observed experimentally. Rather than postulating a conservation of 
Baryon and Lepton number in the MSSM a new symmetry is included into the supersymmetric theory which is called R-parity. R-parity is defined as:

$$
P_{R}=(-1)^{(3 B-L+2 s)},
$$

where B is Baryon number, L is Lepton number and s stands for the spin. All Standard Model particles as well as the Higgs boson have even parity $P_{R}=1$. On the other hand, all supersymmetric particles (sleptons, squarks, gauginos, higgsinos) have odd parity $P_{R}=-1$. If R-parity is exactly conserved, then there is no mixing between particles and the sparticles. This has three interesting consequences:

- The lightest supersymmetric particle (LSP) with $P_{R}=-1$ must be absolutely stable. The LSP can be a good candidate for dark matter.

- Each heavier state must have at least one LSP in the final decay state.

- Supersymmetric particles can only be produced in pairs.

\subsubsection{Particles in the MSSM}

This section will briefly describe some of the new particles that are introduced in the MSSM [12].

- Neutralinos and Charginos: Because of electroweak symmetry breaking in the MSSM Lagrangian the neutral Higgsinos $\left(\tilde{H}_{u}^{0}, \tilde{H}_{d}^{0}\right)$ and the neutral gauginos $(\tilde{B}$, $\tilde{W}^{0}$ ) build four different neutral combinations called neutralinos. The charged Higgsinos $\left(\tilde{H}_{u}^{+}, \tilde{H}_{d}^{-}\right)$and the charged winos $\left(\tilde{W}^{-}, \tilde{W}^{+}\right)$also combine into four states with charge \pm 1 called chargino. In this mass hierarchy the neutralino is a good candidate for the Lightest Supersymmetric Particle (LSP).

- Gluinos: Gluinos build a Majorana fermion color octet similar to their bosonic Standard Model partner the gluon. Gluinos cannot mix with other MSSM particles, even if R-Parity is violated. In most models the gluino is considered to be much heavier than charginos or neutralinos. 
- Squarks and Sleptons: The superpartners of quarks and leptons are all bosonic in nature. This allows particles that are in the same state, which is represented by having the same quantum numbers, to mix with each other. However most of the mixing angles are considered to be very small.

Depending on the chosen model parameters there are several decay channels for the different particles. For this analysis we are mainly interested in multijet final states. This experimental signature, known as well as new physics processes is described in Section 2.4

\subsubsection{R-parity violation}

In the previous discussion of the Minimal Supersymmetric Standard Model the assumption was made that R-parity is a conserved quantity. However, since no sparticle has been observed, R-parity violation (RPV) cannot be excluded.

A violation of R-parity leads to three major differences to the model described above [14].

- Single production of supersymmetric particles is possible.

- The LSP is not necessarily stable. Therefore, it can decay further into normal matter, for example.

- Lepton or Baryon number is violated.

These new properties make it possible to search for supersymmetric signals that decay exclusively into Standard Model particles. We test a model of the production of R-parity violating gluinos decaying into three partons which is described in Section 2.4 .2 


\subsection{Multijet Signals}

The analysis presented in this thesis is a model independent search for a pair produced new hadronic resonance decaying into three partons. These partons can't be observed as single particles and form jets. A jet is the product of the hadronization process of the outgoing partons and is mainly identified by its energy deposition in the calorimeter of the detector. Jets are described in more detail in Section 4.1.1. One of the main requirements is that the final state consists of at least 6 jets. All multijet physics suffers from large QCD backgrounds due to the much larger cross sections of these processes. To test the analysis technique we use known Standard Model decays as well as a model for new physics that results in multijet final states. We discuss the top quark decay as a Standard Model particle which has a decay channel that is similar to the signature we are looking for. The all-hadronic top quark decay is used to test the analysis technique and its contribution is included into our background model.

Most jets originated by QCD events are gluon jets, whereas jets originated from a real resonance are quark jets. QCD final states with high jet multiplicity are theoretically very difficult to calculate. In this analysis we present a technique to extract a multijet resonance from the QCD background by using a data driven background procedure.

\subsubsection{Top Quark}

The top quark was discovered in 1995 simultaneously by the CDF and D $\phi$ collaborations at the Tevatron. It was theoretically predicted to complement the isospin doublet of the bottom quark. With a mass of $172.4 \mathrm{GeV} / \mathrm{c}^{2}$ it is the heaviest observed particle. The top has an extremely short lifetime of only $\tau=10^{-25} s$ which allows us to study its properties before it undergoes the process of hadronization. At the Tevatron it is produced as $t \bar{t}$ by quark-antiquark annihilation with a probability referred to as branching ratio $\mathrm{BR}=85 \%$ and gluon fusion $\mathrm{BR}=15 \%$ [15]. It decays weakly almost exclusively into a $\mathrm{W}$ boson and a b quark. 
The three different decay channels of a $t \bar{t}$ pair are:

- Leptonic decay: $\left(t \bar{t} \rightarrow W^{+} b W^{-} \bar{b} \rightarrow \bar{l} \nu_{l} b l^{\prime} \overline{\nu_{l^{\prime}}} \bar{b}\right)$ Both $\mathrm{W}$ bosons decay into leptons and lepton-anti-neutrinos. The branching ratio for this decay is $\mathrm{BR}=10.3$ \%. The experimental signature is therefore two leptons, two jets and missing $E_{T}$.

- Semi-Leptonic decay: $\left(t \bar{t} \rightarrow W^{+} b W^{-} \bar{b} \rightarrow q \overline{q^{\prime}} b l^{\prime} \overline{\nu^{\prime}} \bar{b}\right)$ In this case only one of the $\mathrm{W}$ bosons decays leptonically while the other one decays into quarks. The branching ratio is $\mathrm{BR}=43.5 \%$. The experimental signature is one lepton, four jets and missing $E_{T}$.

- All-hadronic decay: $\left(t \bar{t} \rightarrow W^{+} b W^{-} \bar{b} \rightarrow q \bar{q}^{\prime} b q^{\prime \prime} \bar{q}^{\prime \prime \prime} \bar{b}\right)$ The most common decay is the all-hadronic channel, where both $\mathrm{W}$ bosons decay into quarks. The branching ratio is $\mathrm{BR}=46.2 \%$. The final state can be seen in the detector as an event with at least six jets.

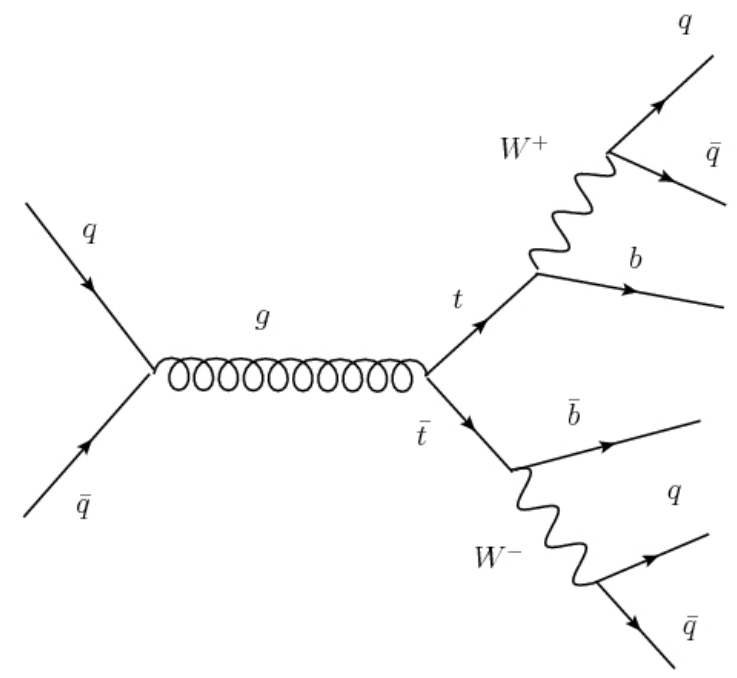

Figure 2.6: $t \bar{t}$ decay in the all-hadronic channel resulting in at least 6 jets 


\subsubsection{RPV Gluino}

Even though we perform a model independent search for a new hadronic resonance, we choose an R-parity violating supersymmetric scenario as a generic model of the signal. We generate the signal as a gluino pair where each of the two particles decays into three jets. The decay chain has an intermediate squark with a mass between $500 \mathrm{GeV} / c^{2}$ and $700 \mathrm{GeV} / c^{2}$. The decay is shown in Figure 2.7. However, an intermediate resonance is not required for this analysis, but might help to further distinguish between background and signal.

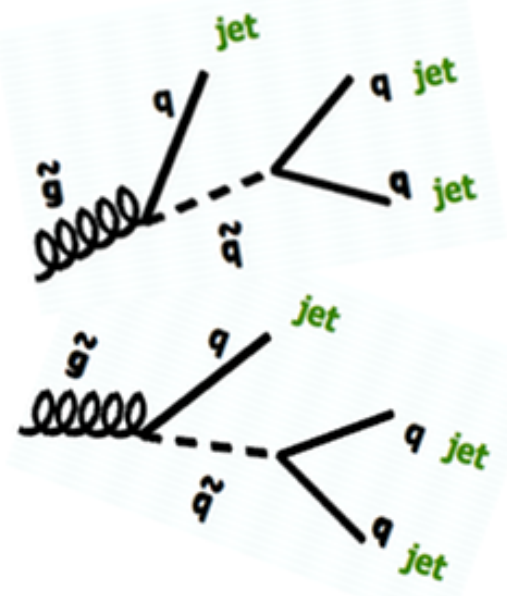

Figure 2.7: Pair produced gluinos decaying into three jets each 


\section{Chapter 3}

\section{The experimental apparatus}

\subsection{The Tevatron Complex}

The Tevatron particle accelerator complex is located at Fermilab in Illinois, and produces high energy particle collisions. A diagram of the complex is shown in Figure 3.1 . The Tevatron collides protons $(\mathrm{p})$ and antiprotons $(\bar{p})$ at a center-of-mass energy of $1.96 \mathrm{TeV}$. We can divide the lifetime of the experiments into two ranges Run $\mathrm{I}(\sqrt{s}=$ $1.8 \mathrm{TeV})$ and Run II $(\sqrt{s}=1.96 \mathrm{TeV})$. Major changes took place between these two periods and we use data from the latter in this thesis. By 2010 the collider produced 8 $f b^{-1}$ of data which is limited by the number of $\bar{p}$ since their production is a challenging process. The Tevatron is planned to run until 2011. The whole accelerator complex can be divided up into different sections which will be described below.

\subsubsection{Proton source}

The protons are produced by using an ionized gas of hydrogen $\left(H^{-}\right)$in a pre-accelerator with a Cockcroft-Walton design [17]. An electric field of $750 \mathrm{keV}$ is applied which accelerates the ions. Then the particles are fed into a $400 \mathrm{MeV}$ linear accelerator before they finally reach an $8 \mathrm{GeV}$ booster synchrotron. As the hydrogen ions are injected into this synchrotron they pass a carbon foil where the two electrons are ripped away and only the proton enters the booster. After reaching $8 \mathrm{GeV}$ the protons are transferred to the Main Injector. 
FERMILAB'S ACCELERATOR CHAIN

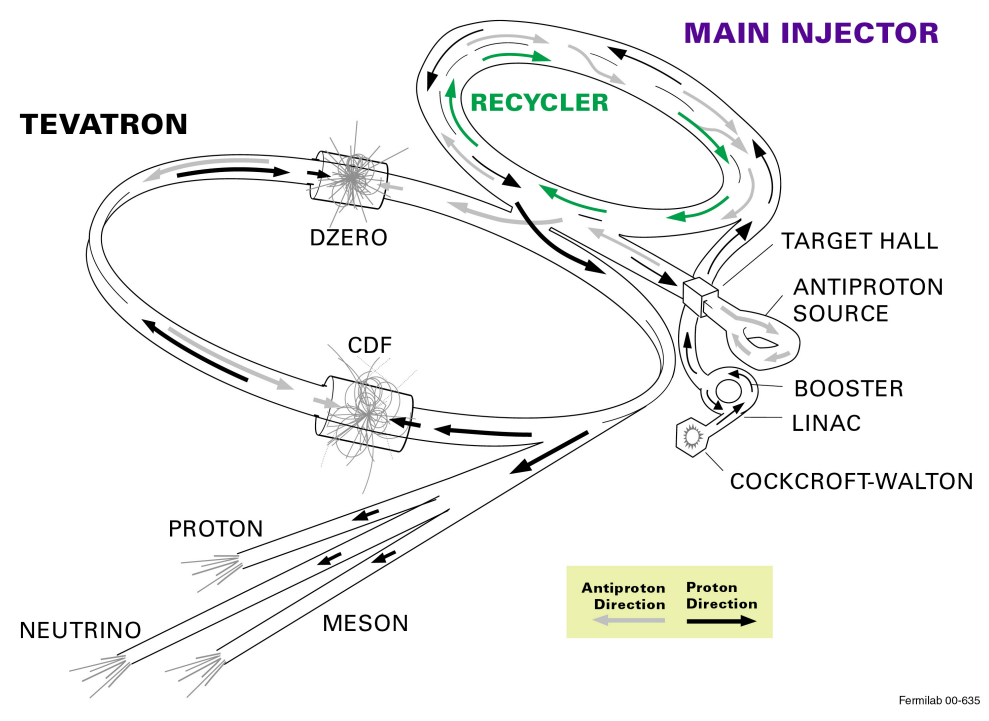

Figure 3.1: Tevatron Accelerator complex at Fermilab [16]

\subsubsection{Main injector}

The Main Injector is a synchrotron used for further acceleration as well as for antiproton production. Protons coming from the booster are accelerated to an energy of $150 \mathrm{GeV}$ [18] before injecting them into the main collider. In order to produce antiprotons the protons reach an energy of $120 \mathrm{GeV}$ and are then guided into a fixed target experiment.

\subsubsection{The Antiproton source}

The main limiting factor for the luminosity and collision rate at the Tevatron is the production of antiprotons. Antiprotons are produced with a $120 \mathrm{GeV}$ proton beam coming from the main injector and colliding into a nickel target. As a result of this collision a shower of particles is produced which is collimated with a lithium lens. In order to only keep the negatively charged particles a dipole magnet is used to select them. The dipole magnet is pulsed to pick out antiprotons with an energy of $8 \mathrm{GeV}$. This happens with an efficiency of 1 antiproton per 10000 protons [19]. This production mechanism leads to a particle beam with a wide range of momentum. For efficient collisions we need a small phase space volume and to achieve this the antiprotons need 
to be cooled. This happens partially already in the main injector as well as in the accumulator, where the particles are stored before injecting them into the Tevatron. Figure 3.1 also shows a part of the Tevatron which is called Recycler. Since it is difficult and costly to produce antiprotons the main purpose of the Recycler is to store them after they have been used in the Tevatron. The Recycler holds them at $8 \mathrm{GeV}$ until the next store of the collider.

\subsubsection{The Tevatron}

After producing enough protons and antiprotons they are accelerated in the Main Injector up to $150 \mathrm{GeV}$ before they are fed into the Tevatron. The particle accelerator is a $2 \mathrm{~km}$ diameter synchrotron and consists of superconducting magnets with eight accelerating cavities. In order to use the superconducting magnets the temperature is brought down to $4 \mathrm{~K}[20$. It accelerates the protons and antiprotons from $150 \mathrm{GeV}$ up to 980 $\mathrm{GeV}$. The Tevatron works as a storage ring and only brings the beams together to collide at the places where the main experiments are being conducted. These experiments are namely $\mathrm{D} \phi$ and CDF (Collider Detector at Fermilab). For this analysis we use data from the CDF Run II detector which is described in the next section. Quadrupole magnets are used to squeeze the beam when it reaches the area of the experiments in order to obtain a higher luminosity. The instantaneous luminosity is described by

$$
L=\frac{f N_{B} N_{p} N_{\bar{p}}}{2 \pi\left(\sigma_{p}^{2}+\sigma_{\bar{p}}^{2}\right)}
$$

and is a measure for the number of collisions that occur in a time interval per area in $\mathrm{cm}^{2}$. The following variables are used in equation $3.1 . N_{B}$ is the number of bunches in the beam, $\mathrm{f}$ is the frequency of bunch crossings, $N_{p}, N_{\bar{p}}$ describe the number of protons and antiprotons and $\sigma_{p}, \sigma_{\bar{p}}$ describe the beamsize of the colliding beam in the transverse plane [19]. 


\subsection{CDF - Collider Detector at Fermilab}

CDF is one of the two multipurpose experiments located at the Tevatron accelerator ring. We only use data from the Run II phase of the experiment therefore in the next section only the upgraded version is described. An elevation view of half of the CDF Run II detector is shown in Figure 3.2 . The detector is centered around the beamline and the closest part to the beamline is the tracking system which is necessary to reconstruct the path of charged particles that are produced in the collision. A $1.4 \mathrm{~T}$ magnet whose field points in the beam direction force particles to describe a helix structure while they move through the tracking system. In order to measure the energy of the particles as precisely as possible, a tower geometry was chosen for the electromagnetic and hadronic calorimeters [21]. To measure the muons which are minimum ionizing particles the outermost parts of the detector are drift chambers. The detector elements are described in detail below.

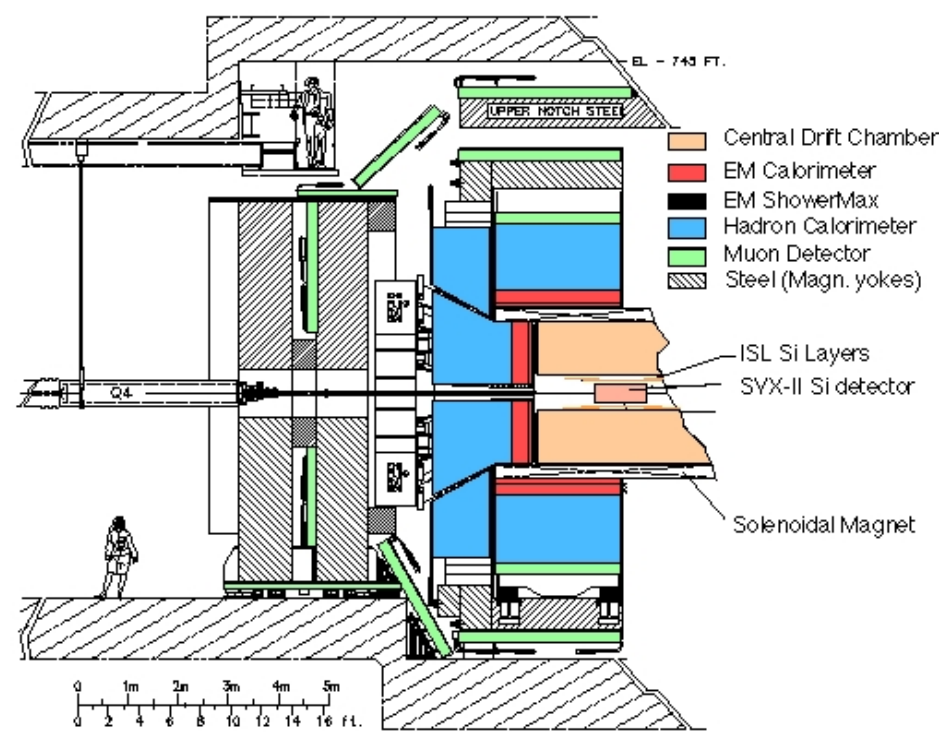

Figure 3.2: Elevation view of half of the CDF Run II detector [22] 


\subsubsection{Detector Coordinates}

It is important to define a coordinate system that is suitable for the geometry of the detector. A spherical coordinate system with $(\mathrm{r}, \theta, \phi)$ is used to describe a position within the detector. The three variables can be defined in cartesian coordinates (equation 3.2 -3.4 :

$$
\begin{gathered}
r=\sqrt{x^{2}+y^{2}+z^{2}}, \\
\theta=\cos ^{-1}\left(\frac{z}{r}\right), \\
\phi=\tan ^{-1}\left(\frac{y}{x}\right) .
\end{gathered}
$$

At a center of mass energy of $1.96 \mathrm{TeV}$ we have to consider relativistic invariant coordinates which cannot be satisfied by the above mentioned ones. A boost along the $\mathrm{z}$ axis might occur during the collision therefore we define the Lorentz invariant rapidity as in in equation 3.5

$$
y=-\frac{1}{2} \log \frac{p+p_{z}}{p-p_{z}} .
$$

Equation 3.5 can be simplified to 3.6 the so-called pseudo rapidity if the mass of the particle is either 0 or negligible.

$$
\eta=-\log \tan \frac{\theta}{2}
$$

Since particles might be boosted along the $\mathrm{z}$ axis in the detector the transverse plane gives a framework for invariant variables. We can define transverse momentum $p_{T}$ and transverse energy $E_{T}$

$$
\begin{gathered}
p_{T}=p \sin \theta \\
E_{T}=E \sin \theta .
\end{gathered}
$$




\subsubsection{Tracking System}

The tracking system is used to determine the trajectory of the charged particles. As shown in Figure 3.3 the system contains two major parts; the silicon detector (Layer 00, Silicon Vertex Detector (SVX), Intermediate Silicon layers) and an open-cell drift chamber (COT - Central Outer Tracker). This combination provides tracking out to $|\eta|<2$.

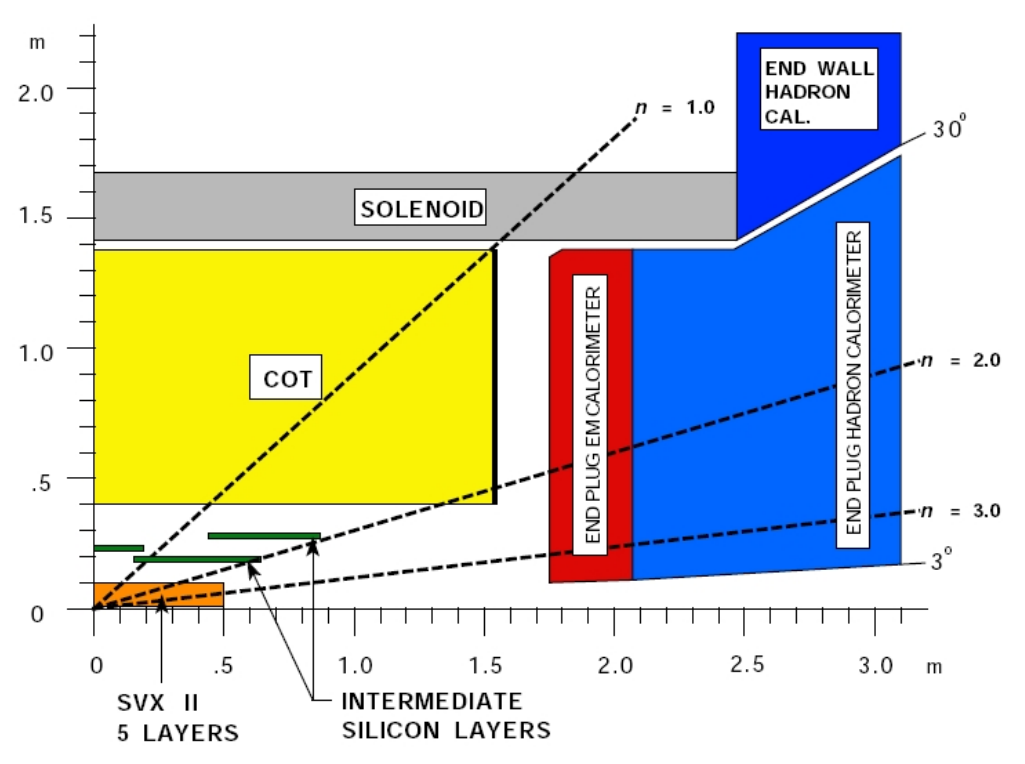

Figure 3.3: CDF Tracking System [23]

Since silicon is a semiconductor material it works well for detecting tracks of particles. When a charged particle moves through the material it produces electron-hole pairs which can be measured as a current if a voltage is applied. The main hit information comes from the SVX which is positioned at radii of 2.4 to $10.7 \mathrm{~cm}$ away from the beamline. It is constructed of three barrels divided into 12 wedges in $\phi$. Three concentric detectors, made out of silicon, are placed outside the beamline. These detectors cover an area of $|\eta|<2$. Because of the already mentioned magnetic field the particles describe a helix path in the tracking system. When we go further away from the beamline the silicon is replaced by an open-cell drift chamber called central outer tracker (COT). The COT is filled with a mixture of 50\% argon and 50\% ethane. Eight 
superlayers of $310 \mathrm{~cm}$ length cells are placed at radii of $40 \mathrm{~cm}$ and $132 \mathrm{~cm}$. Each superlayer has 12 layers of sense wires. The eight superlayers can be divided further into axial layers which are parallel to the beam axis in order to measure the particle track in the transverse plane and so-called stereo layers. The stereo layers are positioned at $\pm 2^{\circ}$ with respect to the beam axis to measure tracks in the $\mathrm{z}$ direction [24]. Over 30,000 read-out channels and a hit resolution of $180 \mu \mathrm{m}$ make the reconstruction of particle tracks possible. When charged particles cross the drift chamber they ionize the gas. The free electrons drift to the wires when a potential is applied. The readout electronics can now measure the charge of the signal.

\subsubsection{Calorimeters}

To measure the energies of the outgoing particles a system of electromagnetic and hadronic calorimeters is used. The energy resolution in the calorimeter is a major limitation in high energy particle physics. A good understanding of the interaction of charged and neutral particles in matter is therefore crucial.

\section{Electromagnetic Calorimeter}

Electromagnetic calorimeters are usually made of a heavy material which causes the charged particles to produce an electromagnetic shower due to bremsstrahlung and pair production. At $\mathrm{CDF}$ the electromagnetic calorimeter is a sampling calorimeter with alternating layers of lead and scintillator. When an electron enters the medium it is deflected from its original path due to interactions with the atomic field in the material, this causes the emission of bremsstrahlung. The cycle of bremsstrahlung producing photons and photons producing $e^{+} e^{-}$pairs continues until the threshold energy for pair production is reached. The radiation length $\left(X_{0}\right)$ is a characteristic length that describes the energy loss of an electron beam in a material. It has a strong inverse dependence on $\mathrm{Z}$, the atomic number of the material, and gives the mean distance an electron travels in a material until it looses all but $\frac{1}{e}$ of its energy [25]. With this method the energy of the particle is measured by photomultipliers which translate the photons produced into a signal which represents the total energy. The energy resolution for a 
particle passing through the electromagnetic calorimeter is given by:

$$
\frac{\sigma_{E}}{E}=\frac{a}{\sqrt{E}} \oplus b \oplus \frac{c}{E},
$$

where $\oplus$ represents the addition in quadrature. The first term parametrized by $a$ represents statistical fluctuation in the energy deposition. The second term $b$ does not depend on the energy and is due to detector effects. The last term $c$ is due to electronic noise in the readout channels [25].

\section{Hadronic Calorimeter}

To measure the energy of hadronic particles a hadronic calorimeter is used. At CDF the hadronic calorimeter is built of alternating layers of iron and scintillator. An important parameter in this case is the nuclear interaction length $\lambda$. Similar to electrons going through an electromagnetic calorimeter, neutrons and pions, for example, interact with the nuclei in the hadronic calorimeter through the strong force and cause an electromagnetic shower to develop in the calorimeter. The nuclear interaction length gives a measure similar to the radiation length.

At CDF a tower based geometry is used and can be divided into a central barrel calorimeter $(|\eta|<1)$ and the forward and end the plug calorimeter $(1.1<|\eta|<3.64)$. The central barrel calorimeter consists of the inner electromagnetic calorimeter (CEM) and an outer hadronic calorimeter (CHA). To cover the region of $0.6<|\eta|<1.0$ a so-called end-wall hadronic calorimeter (WHA) is installed. The plug electromagnetic calorimeter (PEM) and the plug hadronic calorimeter (PHA) cover the region of $1.1<|\eta|<3.6$. 


\subsubsection{Muon detectors}

The last and outermost part of the detector is the muon chamber. Muons are minimum ionizing particles and therefore pass the tracking and calorimeter system almost undisturbed. To prevent hadrons which passed all previous calorimeters from entering the muon chamber a layer of steel is located between the two. The muon chamber is divided into several parts the central muon detector (CMU), central muon extension (CMX), central muon upgrade (CMP) and the intermediate muon detector (IMU). The CMU contains 2304 single-wire drift chambers which are arranged in four concentric radial layers and covers a range in the detector of $|\eta|<0.6$. To reach the CMU the muon needs a minimum $p_{T}$ of $1.4 \mathrm{GeV} / \mathrm{c}$. The CMP is located directly after the CMU in a radial direction and to reach this part of the muon chamber the $p_{T}$ threshold is raised to $2.2 \mathrm{GeV} / \mathrm{c}$. The total coverage of all four parts of the muon chambers is for $|\eta|<2.0[24]$.

\subsubsection{Trigger System}

At the Tevatron the time between two bunch crossings and therefore theoretically two events is $392 \mathrm{~ns}$, or a frequency of $2.5 \mathrm{MHz}$ [19]. To store every single one of these events would not just be a huge challenge for the electronics but also would not be useful for analyses since only a fraction of the events are physically interesting. CDF uses a three level trigger system which includes several hardware and software components [26].

- Level 1 is a hardware trigger that selects events based on calorimeter, tracking and muon chamber information. It reduces an input rate of $1.7 \mathrm{MHz}$ to an output rate of roughly $20 \mathrm{kHz}$. At level 1 electrons and jets are defined as single-tower energy depositions in the calorimeters.

- Level 2 is an asynchronous combination of hardware and software triggers. It starts a simple event reconstruction adding more information to the events that passed Level 1 and starts clustering energy depositions in the calorimeter. The average processing time at level 2 is about $30 \mu s$. 
- Level 3 is a offline software trigger. At this stage the complete event is reconstructed. A loose set of selection cuts is applied to the event and the output rate is brought down to $75 \mathrm{~Hz}$. These events are recorded and saved for further analysis.

Since CDF is a multipurpose detector which is looking for all kinds of interesting physics processes the data can be divided into different datasets which followed different trigger paths that are optimal for a particular analysis.

\subsection{Datasets}

We are using a dataset corresponding to the integrated luminosity of:

$$
\int L d t=3.2 f b^{-1}
$$

The trigger used in this analysis is the so-called TOP_MULTIJET_TRIGGER and was originally invented to study the all-hadronic $t \bar{t}$ decay. It requires at least 4 high $p_{T}$ jets and a large $\sum_{4 j} E_{T}[27]$ :

- Level 1: The trigger requires at least 4 distinct energy clusters in the electromagnetic or hadronic calorimeters with a raw energy greater than $10 \mathrm{GeV}$.

- Level 2: At this level the energy threshold is raised to $15 \mathrm{GeV}$ for the at least 4 jets. The sum $E_{T}$ of all jets has to be greater than $175 \mathrm{GeV}$.

- Level 3: After the full event reconstruction the 0.4 cone jets have to have $E_{T}>15$ $\mathrm{GeV}$.

A simulation for this trigger is available and is incorporated into our Monte Carlo simulation following Reference [27]. Each event is weighted according to this trigger simulation.

\subsection{Monte Carlo}

In order to test analysis techniques and optimize searches for signals, Monte Carlo simulations are used. Monte Carlo generators such as PYTHIA [28] use the underlying 


\begin{tabular}{|c|c|c|c|}
\hline$\overline{M S}_{\tilde{g}}$ & $M_{\tilde{g}}$ & Cross Section $(p b)$ & k-factor \\
\hline $50 \mathrm{GeV} / c^{2}$ & 73.7 & 1922 & 1.74 \\
$80 \mathrm{GeV} / c^{2}$ & 110.1 & 205.5 & 1.72 \\
$100 \mathrm{GeV} / c^{2}$ & 133.5 & 38.9 & 1.66 \\
$130 \mathrm{GeV} / c^{2}$ & 167.9 & 13.2 & 1.56 \\
$150 \mathrm{GeV} / c^{2}$ & 190.3 & 6.6 & 1.52 \\
$180 \mathrm{GeV} / c^{2}$ & 223.3 & 2.3 & 1.47 \\
$200 \mathrm{GeV} / c^{2}$ & 245.0 & 1.2 & 1.45 \\
\hline
\end{tabular}

Table 3.1: Information about signal MC gluino samples.

quantum field theory described by the Standard Model to simulate numerically what happens during a collision of high energy protons and antiprotons. To include the response of the detector to the produced signal other programs such as GEANT [29] are used to model the passage of particles through the different parts of the detector.

We model the signal using PYTHIA, generating pair produced gluino events with hadronic RPV turned on (hadronic uds Matrix Element, which allows gluino decays to light jets). To execute actual calculations PYTHIA chooses a specific renormalization scheme which is necessary to cancel infinities arising in quantum field theory. This is the so-called $\overline{M S}$ scheme and the $\overline{M S}$ mass is a parameter which can be translated into the real physical observable mass [3]. PYTHIA is a leading order (LO) Monte Carlo generator, to account for corrections to the cross sections and including next to leading order (NLO) effects a multiplicative factor called the k-factor can be calculated. The first column in Table 3.1 represents the $\overline{M S}$ mass, the second column represents the real physical mass, the third column shows the cross section and the last column represents the k-factor. To study the contribution of the $t \bar{t}$ all-hadronic decay as a background in our analysis we use PYTHIA and perform cross checks with ALPGEN [30] and MC@NLO [31]. To estimate the QCD background we do not use Monte Carlo simulations but choose a data driven background procedure instead. 


\title{
Chapter 4
}

\begin{abstract}
Analysis
This analysis shows a model independent search for 3-jet hadronic resonances in $3.2 \mathrm{fb}^{-1}$ of data at the CDF detector at Fermilab. In order to model new physics signatures, we choose R-parity violating supersymmetric (RPV SUSY) gluinos produced in pairs and decaying into three partons. We measure these partons as jets and use the kinematic quantities and correlations. We create an ensemble of jet combinations which allows us to extract signal from the multijet QCD background [32]. Since the all-hadronic $t \bar{t}$ decay channel has a signature similar to our signal we study this known Standard Model process as well. The analysis is documented as an internal CDF note which can be found in Reference [33]. It has been approved by the CDF collaboration and a public note [34] as well as a website [35] have been released.
\end{abstract}

\subsection{Event Reconstruction}

\subsubsection{Jets}

The experimental reconstruction and theoretical understanding of jets are the foundation of this analysis. Our current understanding of the hadronization process is described in Section 2.2.2 and experimental reconstruction of jets is described in more detail in the next few sections. The signature of a jet in a detector is a shower of particles which is represented by clusters of energy in the electromagnetic and hadronic calorimeters. Since a jet is a shower of different particles, a set of selection rules is needed to define a jet itself, in other words a jet algorithm. There are different approaches to jet algorithms, such as cone and $k_{T}$ algorithms [36]. The one used at CDF and for this analysis is a cone algorithm called JetClu [37] which is described in the 
following section.

\section{JetClu Algorithm at CDF}

- First a list of seed towers in the calorimeter with $E_{T}>1 \mathrm{GeV}$ is created where the transverse energy is defined as:

$$
E_{T}=E_{E M} \sin \theta_{E M}+E_{H A D} \sin \theta_{H A D}
$$

with EM and HAD defining electromagnetic or hadronic calorimeter, respectively [37.

- The list obtained in the first step is ordered by $E_{T}$ and a "precluster" is built by joining adjacent seed towers within a cone in $\eta-\phi$ space starting at the highest $E_{T}$ seed.

- For each precluster the $E_{T}$-weighted centroid is calculated and a cone of radius $\mathrm{R}$ is drawn around it. This radius $\mathrm{R}$ is defined as $R=\sqrt{\left(\eta^{\text {tower }}-\eta^{\text {jet }}\right)^{2}+\left(\phi^{\text {tower }}-\phi^{\text {jet }}\right)^{2}}$ where the coordinates $\left(\eta^{j e t}, \phi^{j e t}\right)$ are defined as the center of the jet [38]. Each cluster consists of towers within $\mathrm{R}$ that have $E_{T}>100 \mathrm{MeV}$. Next an iterative process begins where towers are added to the cluster and the new centroid is calculated after each step. This is done until no new towers are added anymore or the number of steps reached a predefined limit.

- Overlapping of two clusters might happen and if the overlap fraction is $>75 \%$ the two clusters are merged.

- The final list of towers can be regarded as the jet.

For this analysis we use jets with a cone size of $\mathrm{R}=0.4$.

At this stage there are no tracks associated with the jet. In order to reproduce the original parton energy as close as possible several corrections need to be applied to the raw jet energy. 


\section{Jet energy corrections}

To calibrate the energy of the jet to that of the original parton several corrections need to be applied to the raw jet energy measured by the JetClu algorithm which shall be described here briefly and can be seen in further detail in [38].

- $\eta$-dependent corrections: The response of the CDF detector is not uniform over the entire $\eta$ range. This non uniformity is due to the structure of the calorimeter at $\eta=0$, where two halves of the central calorimeter join, and $\eta=1.1$ where plug and central calorimeter join. These cuts in $\eta$ as well as different resolutions in the plug and central calorimeters need to be corrected to ensure an $\eta$ independent energy reconstruction. A "dijet-balancing method" which is described in more detail in [38] is used for this correction.

- Absolute Scale: This is a correction that is derived from Monte Carlo simulations of the detector response and can be parametrized as a function of $p_{T}$. This method relies on how well the jet is modeled by the simulations (particle multiplicity in the jet, $p_{T}$ distribution of the constituents). This correction makes the jet energy independent of the CDF detector.

- Multiple Interactions: In each bunch crossing it is statistically unlikely to only have one $p \bar{p}$ collision and the probability depends mainly on the instantaneous luminosity. Hadrons that are from another $p \bar{p}$ collision inflate the measured jet energy and therefore have to be subtracted. This correction is described as a function of the number of vertices in an event.

- Out-of-cone Correction: The final goal of jet reconstruction is to measure the energy of the original parton as a decay product of a resonance. Due to radiation losses in the final state (FSR) or particles leaving the cone in the hadronization process the measured jet energy might be smaller than the original parent parton. The out-of-cone correction (OOC) is obtained from matching Monte Carlo parent partons with reconstructed jets in the detector.

- Underlying Event: Beside the OOC another process called Underlying Event 
(UE) might cause the measured jet energy be too high compared to the original parton due to initial state radiation (ISR). During a bunch crossing interaction between partons that do not participate in the hard collision might occur. This as well as ISR and FSR are called underlying event. The UE correction is obtained from Monte Carlo.

- Test of jet corrections: Since some of the above mentioned corrections rely on Monte Carlo a cross check with data is necessary to verify that all of them work properly. The corrections are applied to $\gamma-$ jet events where the photon $p_{T}$ is measured in the CEM calorimeter with very high precision. Due to the nature of these events the photon should always balance out the jet momentum $\frac{p_{T}^{\gamma}}{p_{T}^{j e t}}=1$. Other useful events are Z-jet events where the $\mathrm{Z}$ decays into two leptons. $\mathrm{A}$ requirement here is that the $\mathrm{Z}$ and the jet are back to back in the detector. This channel suffers less from background than $\gamma-$ jet events. Uncertainties in the jet energy correction can influence the precision of the analysis and need to be included as systematic uncertainties into the final results.

In our analysis all of the above mentioned corrections are applied to $p_{T}$ and $E_{T}$ of the jets and we refer to these corrected values as L7, unless otherwise stated as raw energies or momenta. Uncertainties in these corrections can affect the final result of the analysis and are included as a systematic uncertainty.

\subsubsection{Tracks}

Tracks are a crucial part for the event reconstruction. They are needed in order to define the primary vertex, identify leptons and are useful to reconstruct jets. The track reconstruction algorithm starts with hits in the COT where it tries to find seeds [39]. Seeds consists of hits in 3 consecutive wires that can be extended to a segment. A segment-linking algorithm connects the seeds in each segment throughout the superlayers of the COT followed by a reconstruction through the silicon tracker. Finally the track is completely reconstructed in $\mathrm{r}-\phi$ plane and a $\mathrm{z}$ position for the track can be defined. 


\subsubsection{Primary Vertex}

The primary vertex is the point where the actual collision of $\mathrm{p}$ and $\bar{p}$ takes place. To find this vertex CDF uses the ZVertexFinder algorithm [40]. To find the vertex it takes an error weighted average position on the $\mathrm{z}$ axis as in equation:

$$
z_{0}=\frac{\sum_{i} z_{i}^{0} / \delta_{i}^{2}}{\sum_{i} 1 / \delta_{i}^{2}}
$$

where $z_{i}^{0}$ is the position on the $\mathrm{z}$ axis for each reconstructed track, and $\delta_{i}^{2}$ the error on this position. The quality of a vertex is defined by hits in the silicon tracker and the COT as shown in table 4.1. For this analysis we require between 1-4 quality 12 primary vertices.

\begin{tabular}{cc} 
number of track & quality flag \\
\hline Si-tracks $\geq 3$ & 1 \\
Si-tracks $\geq 6$ & 3 \\
COT-tracks $\geq 1$ & 4 \\
COT-tracks $\geq 2$ & 12 \\
COT-tracks $\geq 4$ & 28 \\
COT-tracks $\geq 6$ & 60
\end{tabular}

Table 4.1: Quality flag for primary vertex - [40]

\subsubsection{Missing Transverse Energy}

Compared to typical searches for new physics missing transverse energy does not play an important role in our analysis. Missing transverse energy is defined as the vector sum of all visible transverse energy in the detector:

$$
E_{T}=-\sum_{i} E_{T}^{i} \hat{n}_{i}=-\sum \overrightarrow{E_{T}}
$$

where $E_{T}$ is defined in equation 4.1 and $\hat{n}_{i}$ is the transverse unit vector that points from the beamline to the calorimeter tower. Jet energy corrections and minimum ionizing particles such as muons influence the measured amount of missing $E_{T}$ in an event. We need to correct the raw missing $E_{T}$ for these effects. We assume an all-hadronic decay of a pair produced particle should have intrinsically little missing $E_{T}$. However, we are still interested in the missing $E_{T}$ distribution as a variable to select events to reduce the background. We require for our events to have $\mathbb{E}_{T}<50 \mathrm{GeV}$. 


\subsection{Event Selection}

\subsubsection{Analysis Strategy}

We are looking for events with a high jet multiplicity and require at least six jets in an event. Out of these six or more jets we use all possible combinations to build triplets of jets, what we call an ensemble of jets. This leads to a minimum of 20 triplets per event. The challenge is to extract the real signal from these possibilities. We calculate the invariant mass of each triplet $M_{j j j}$ as well as the scalar sum of the transverse momentum $\sum_{j j j}\left|p_{T}\right|$. Then we plot both quantities against each other as can be seen in figure 4.1. Using the distribution of $M_{j j j}$ versus $\sum_{j j j}\left|p_{T}\right|$ ensures that we reconstruct the correct combination of jets in some kinematic regime. The incorrect (uncorrelated) triplets tend to have $M_{j j j} \approx \sum_{j j j}\left|p_{T}\right|$ whereas the correct (correlated) triplets produce a horizontal branch in Figure 4.1 at approximately the invariant mass of the signal that is not present for the background. In Monte Carlo the uncorrelated triplets represent combinatorial confusion. In data the uncorrelated triplets include in addition to combinatorial confusion mainly QCD background events.

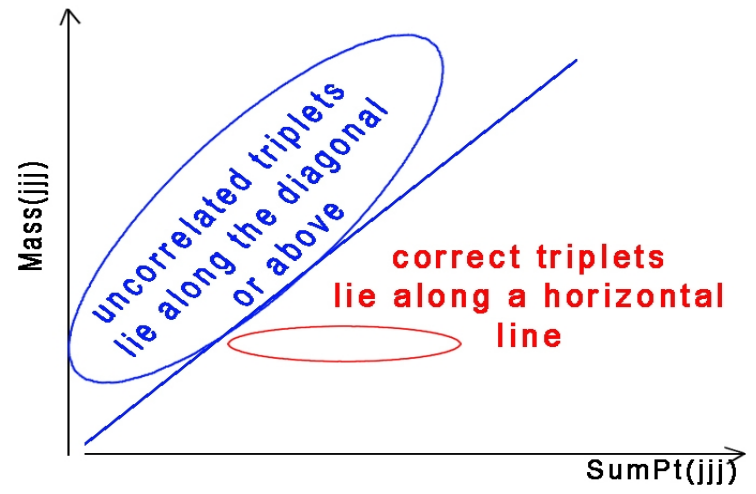

Figure 4.1: $M_{j j j}$ versus $\sum_{j j j}\left|p_{T}\right|$

To extract events along this horizontal branch we select triplets with $\sum_{j j j}\left|p_{T}\right|-$ $M_{j j j}>$ offset. This offset is the intersection of the linear function in Figure 4.1 with the $\sum_{j j j}\left|p_{T}\right|$ axis and depends on the particular mass we are interested in. This offset will be referred to as the "diagonal cut" which is optimized for each mass as described later 
in Section 4.4. Scatter plots for data and different Monte Carlo samples can be seen in figure 4.2 . The $M_{j j j}$ versus $\sum_{j j j}\left|p_{T}\right|$ distributions are shown after applying basic selection criteria described Section 4.2.2 as well as advanced selection criteria described in Section 4.2.3. After applying the diagonal cut we project the events onto the $M_{j j j}$ axis, examples for these final mass plots can be seen in Figure 4.3 .

\subsubsection{Basic Selection}

After the event reconstruction described in Section 4.1 we apply a basic selection on the reconstructed objects. Since we are searching for all-hadronic decays we require that the missing $E_{T}$ in the event does not exceed $50 \mathrm{GeV}$. We require 1-4 quality 12 primary vertices and at least 6 jets in an event. The $p_{T}$ threshold for the energetically lowest jet in the event is set to $15 \mathrm{GeV} / \mathrm{c}$. We require for each jet to be within a distance from the middle of the detector of $\left|z_{0}\right|<60 \mathrm{~cm}$ and finally the $\sum\left|p_{T}\right|$ of the 6 highest jets has to be greater than $250 \mathrm{GeV} / \mathrm{c}$.

- missing $E_{T}<50 \mathrm{GeV}$

- $1 \leq N_{\text {vert }} \leq 4$ (quality 12 vertices)

- $N_{\text {jets }} \geq 6$

- jet $\left|z_{0}\right|<60 \mathrm{~cm}$

- $\sum_{6 j e t}\left|p_{T}\right| \geq 250 \mathrm{GeV} / \mathrm{c}$ for the six highest $p_{T}$ jets in the event.

Examples of these distributions for data and $t \bar{t}$ Monte Carlo can be seen in Figures 4.4 to 4.6 . 

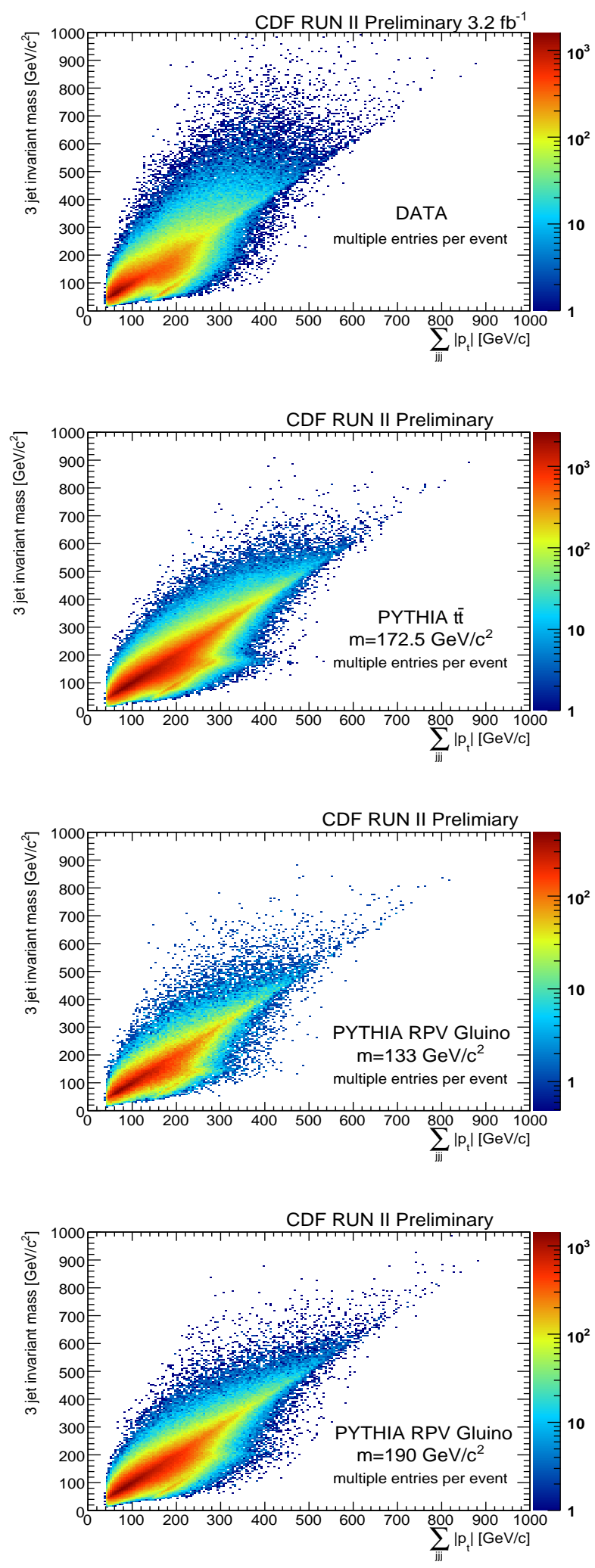

Figure 4.2: $M_{j j j}$ versus $\sum_{j j j}\left|p_{T}\right|$ multiple entry scatter plots. First: data $3.2 f b^{-1}$, Second: PYTHIA $t \bar{t} \mathrm{~m}=172.5 \mathrm{GeV} / \mathrm{c}^{2}$, Third: PYTHIA RPV gluino $\mathrm{m}=133 \mathrm{GeV} / \mathrm{c}^{2}$, Fourth: PYTHIA RPV gluino $\mathrm{m}=190 \mathrm{GeV} / \mathrm{c}^{2}$. All plots have basic and advanced selection criteria applied. To reduce background we apply a loose diagonal cut selection where at least one triplet in the event has to pass a diagonal cut of $100 \mathrm{GeV} / \mathrm{c}$. 

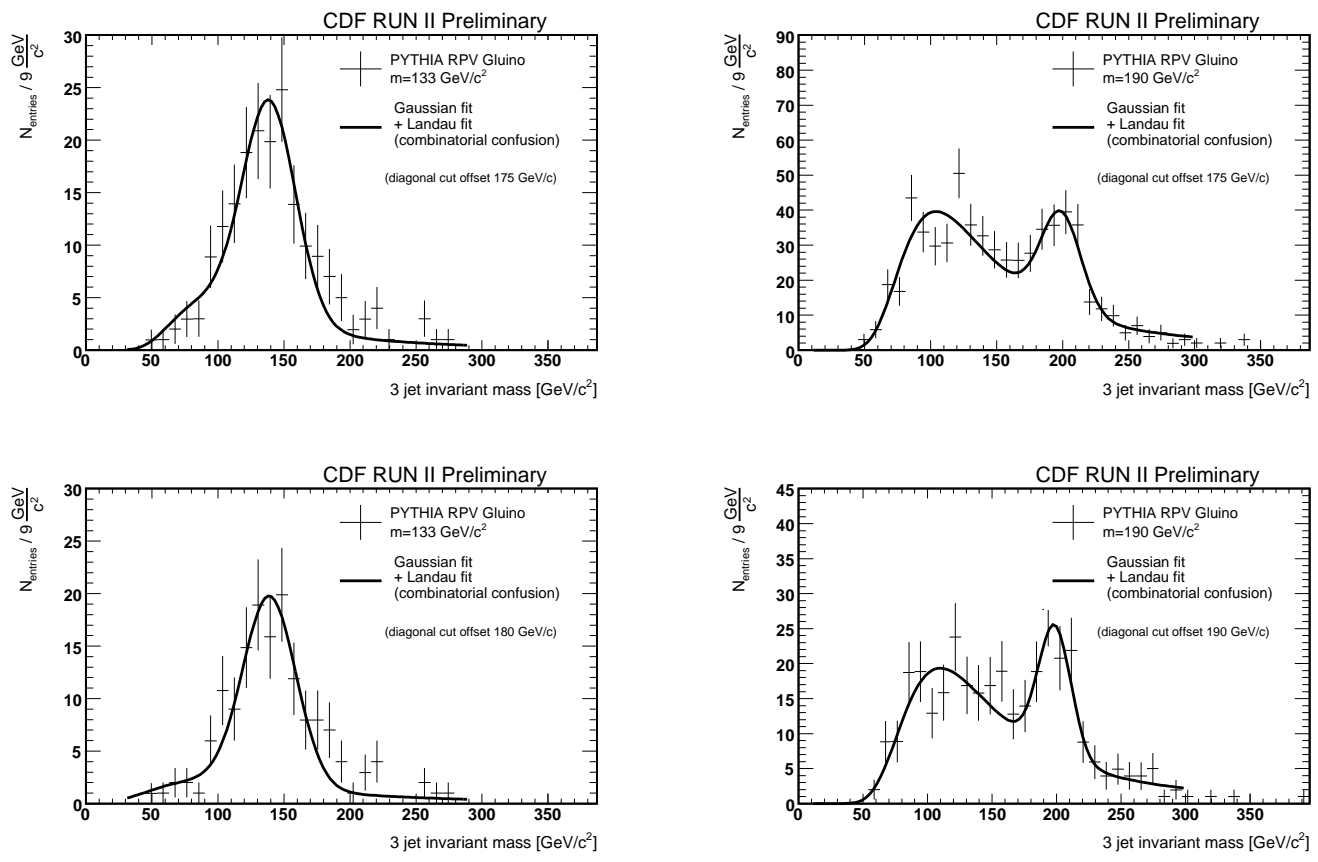

Figure 4.3: These plots show the final mass plot for two different gluino masses and diagonal cuts. The bottom plots show the optimal diagonal cut for each mass. Left: gluino with $\mathrm{m}=133 \mathrm{GeV} / \mathrm{c}^{2}$ the solid line is a fit to Gaussian + Landau (combinatorial confusion), Right: gluino with $\mathrm{m}=190 \mathrm{GeV} / \mathrm{c}^{2}$ the solid line is a fit to Gaussian + Landau (combinatorial confusion) 

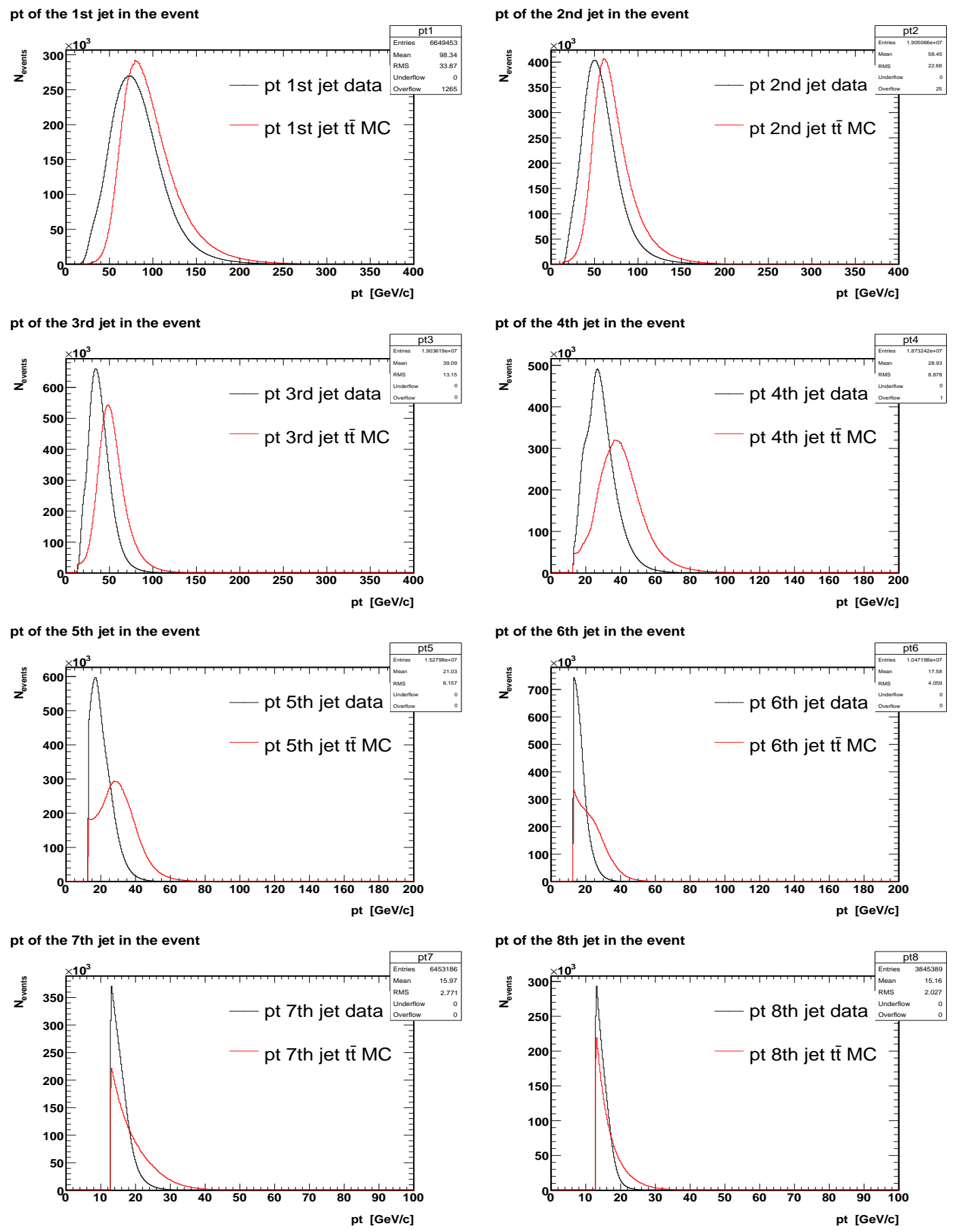

Figure 4.4: $p_{T}$ distribution for the top 8 jets in the data and PYTHIA $t \bar{t}$ Monte Carlo. The jets have L7 jet corrections applied. 

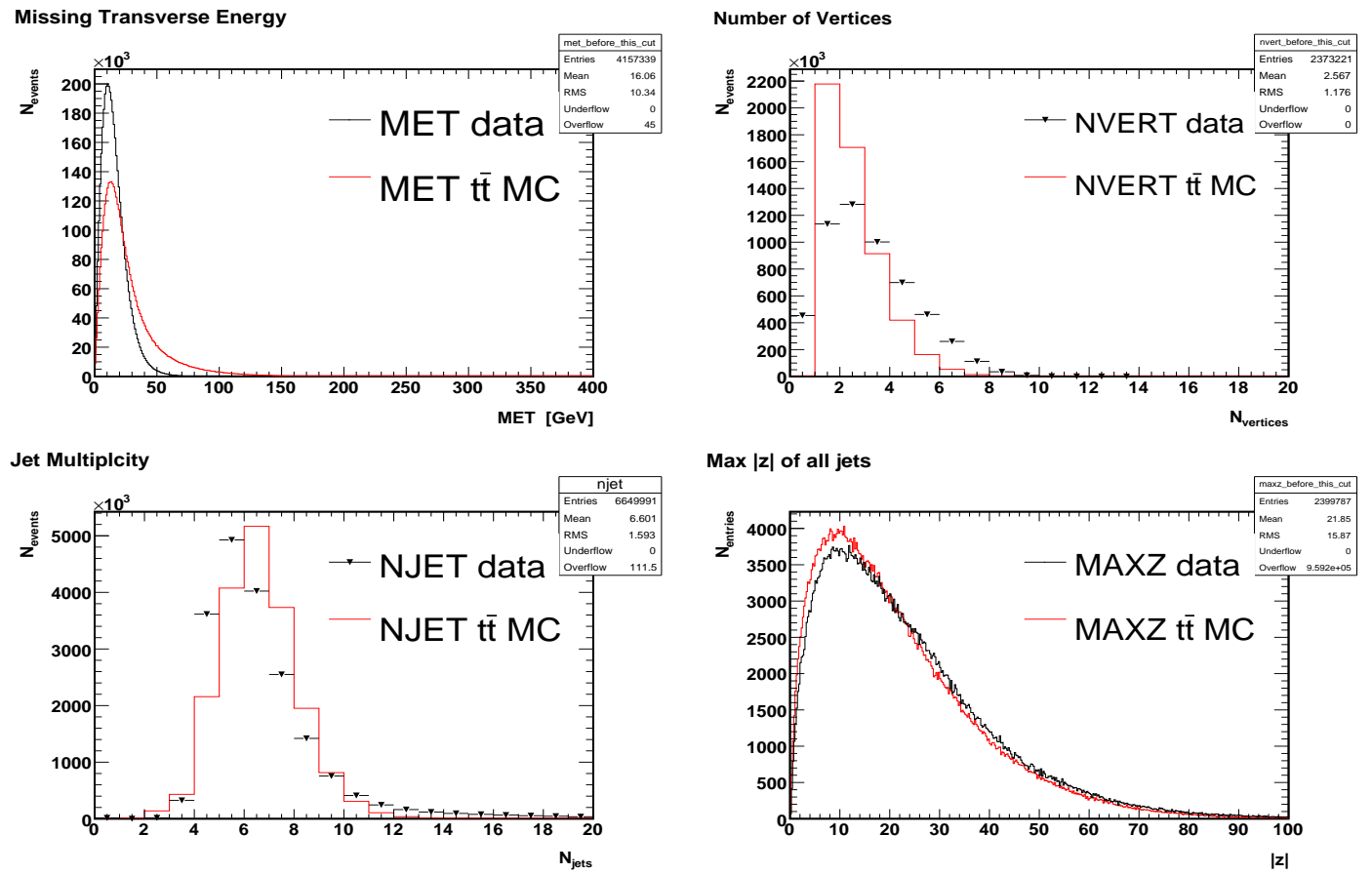

Figure 4.5: Distributions of missing $E_{T}$, number of vertices, number of jets, and the maximum $\left|z_{0}\right|$ of all jets in data and PYTHIA $t \bar{t}$ Monte Carlo before cuts. We require $E_{T} \leq 50 \mathrm{GeV}, 1 \leq \mathrm{Nvert} \leq 4$, Njets $\geq 6$, jet $\left|z_{0}\right|<60 \mathrm{~cm}$.
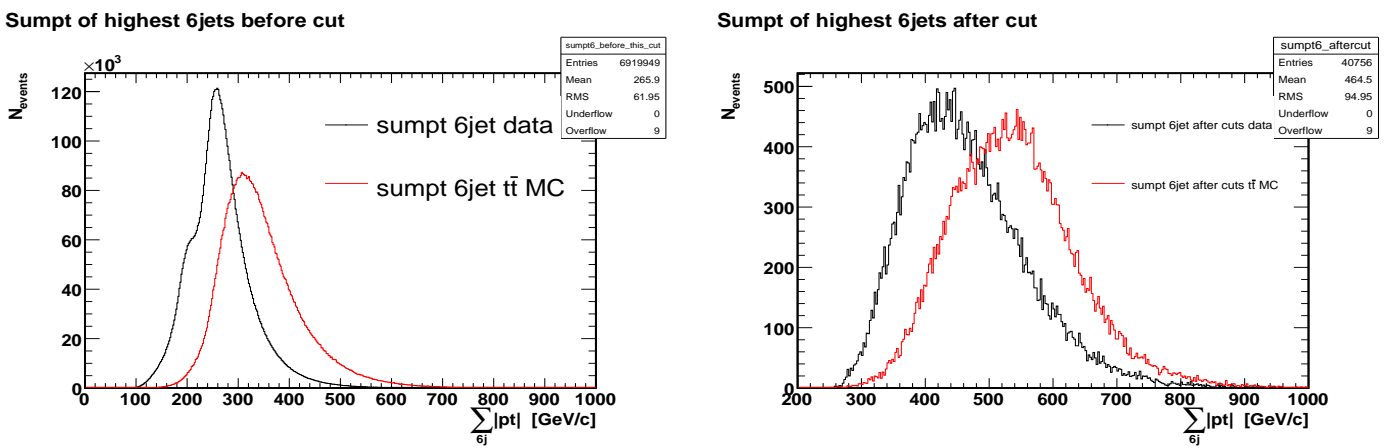

Figure 4.6: $\quad \sum_{6 j e t} p_{T}$ distribution in data and PYTHIA $t \bar{t}$ Monte Carlo before and after after basic and advanced selection and a diagonal cut of $100 \mathrm{GeV} / \mathrm{c}$. We require $\sum_{6 j e t} p_{T} \geq 250 \mathrm{GeV} / \mathrm{c}$ for six highest $p_{T}$ jets. Before we apply our event selection, the effect of the trigger turn-on can be seen. 


\subsubsection{Advanced Selection}

After we apply the above mentioned basic selection to our events we reach the stage where we combine the jets into triplets. By looping over all jets in an event and building every possible combination we obtain an ensemble of at least 20 triplets per event. The number of combinations grows with the number of jets. To ensure that we are really combining jets that come from the same interaction point, the hard scattering, we place a selection on the $z$ position of the jets within an event as well as in a triplet.

- The first step is to demand that all the jets are coming from the same $z$ position. We place a cutoff on $z_{\text {rms }}$ over all jets. To define $z_{\text {rms }}$ we first loop over all tracks associated with the jet and require that $\Delta R_{\text {track-jet }}<0.4$.

- Next we define the mean $\bar{z}_{j}$ position of a jet (obtained from all tracks within the 0.4 cone of the jet) as:

$$
\bar{z}_{j}=\frac{\sum_{\text {tracks }} z_{0}}{N_{\text {tracks }}} .
$$

- The error on $z_{j}$ is defined as:

$$
\delta\left(z_{j}\right)=\sqrt{\frac{\overline{z_{j}^{2}-\bar{z}_{j}^{2}}}{N_{\text {tracks }}}} .
$$

- At this point, each jet that has track information will have the $\mathrm{z}$ information above. Finally, we define $z$ rms as:

$$
z_{\mathrm{rms}}=\sqrt{\frac{\left(\sum_{\text {jets }} \bar{z}_{j}{ }^{2}\right) / N_{\text {jets }}-\left(\sum_{\text {jets }} \bar{z}_{j} / N_{\text {jets }}\right)^{2}}{N_{\text {jets }}}}
$$

Before evaluating $z_{\mathrm{rms}}$ we require that at least 4 of all the $\geq 6$ jets in the event have $\delta\left(z_{j}\right)<4$. We select events with a $z_{\mathrm{rms}}<0.5$. Since jets are not necessarily associated with tracks we allow one jet in the triplet to have no tracking information and therefore no $\mathrm{z}$ information. Jets without any tracks are usually high $\eta$ jets where only calorimeter information is available. 


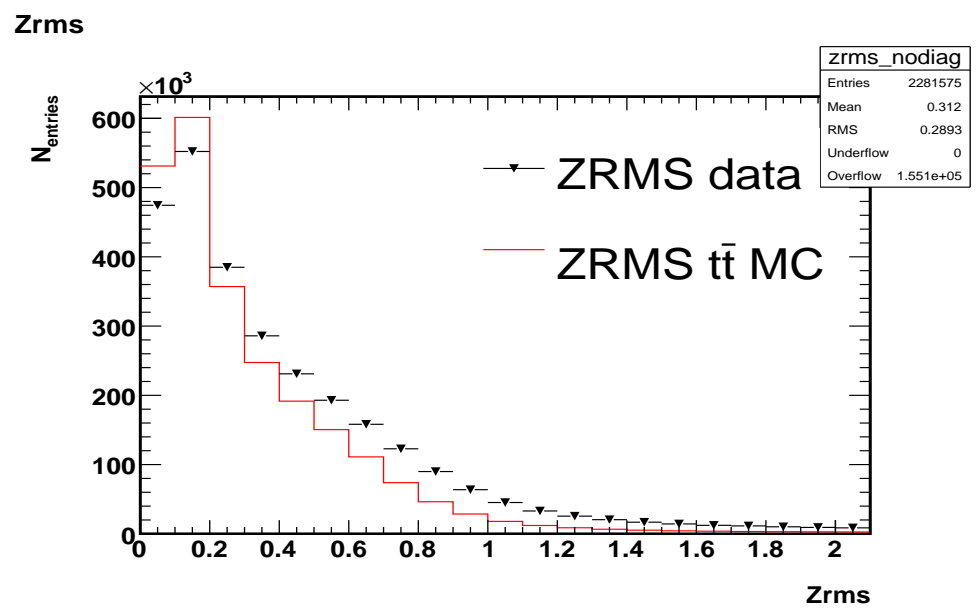

Figure 4.7: $z$ rms distribution in data and PYTHIA $t \bar{t}$ Monte Carlo before the $z_{\text {rms }}$ cut.

For jets in a triplet, we tighten the requirement on the error of the mean z position to $\delta\left(z_{j}\right)<2.5$. Finally, we require that all jets in a triplet have $\left|\bar{z}_{j}-z_{\mathrm{vtx}}\right|<10 \mathrm{~cm}$, where $z_{\mathrm{vtx}}$ is the $z$ position of the first quality class 12 vertex. Triplets that pass all these cuts are what we defined as "good triplets". Figure 4.7 shows the $z_{\text {rms }}$ distribution in the data and $t \bar{t}$ Monte Carlo.

\subsection{Background}

A multijet final state suffers from a large amount of QCD background. QCD processes that occur in a hadron collider are described in Section 2.2. Estimating such a background from Monte Carlo is not only difficult to calculate but it is also impractical to generate a large enough Monte Carlo sample using, for example, a generator such as ALPGEN. Therefore, we need a data-driven background method and Section 4.3.1 describes the technique used in this analysis. The all-hadronic decay of $t \bar{t}$ contributes as a background to our gluino search and the Monte Carlo prediction is included in the background estimate. 


\subsubsection{Data-driven QCD Background estimate}

Our technique for estimating the QCD background does not rely on Monte Carlo but comes directly from data. However, we need to be cautious about fitting for a signal and background in data at the same time, since statistical fluctuations can artificially inflate the signal, while deflating the background. To get around these problems, we use the (statistically independent) exclusive 5-jet data sample to parameterize the shape of the QCD background in the $\geq 6$-jet sample (for brevity referred to as 6-jet). However, before fitting the 5 -jet background we find that we need to scale the $\sum_{j j j}\left|p_{t}\right|$ of the three jets (triplet) in the 5 -jet sample to match the distribution in the 6 -jet sample.

Both $\sum_{j j j}\left|p_{t}\right|$ distributions can be seen in Figure 4.8. To find the correct scale factor, we take the ratio of 6 -jet $\sum_{j j j}\left|p_{t}\right|$ distribution divided by the 5 -jet distribution. The scaling function can be seen in Figure 4.8. The weight function is a fit which is adjusted to the kinematic range where most of the 6-jet events reside. We calculate a scale factor for each triplet according to its triplet $\sum_{j j j}\left|p_{t}\right|$. This scale factor is used to weigh the 5 -jet $M_{j j j}$ distribution.

Although the statistics of the 5-jet sample is smaller, we use it to fit a Landau distribution which seems to describe the shape of the background well. A Landau distribution can be parametrized by three parameters: amplitude, most probable value (MPV) and the width. It is implemented in the math Root library as a numerical interpolation of the integral form described in equation 4.7 .

$$
p(x)=\frac{1}{2 \pi i} \int_{c-i \infty}^{c+i \infty} e^{s \log s+x s} d s .
$$

After fitting the Landau and stepping through diagonal cuts from $100 \mathrm{GeV} / \mathrm{c}$ to 200 $\mathrm{GeV} / \mathrm{c}$ in $1 \mathrm{GeV} / \mathrm{c}$ steps we save the amplitude, MPV and width of the Landau function for each diagonal cut. Examples for fits at different diagonal cuts $(134 \mathrm{GeV} / \mathrm{c}, 155$ $\mathrm{GeV} / \mathrm{c}, 165 \mathrm{GeV} / \mathrm{c}$ ) are shown in figure 4.9. We note that all three values can be parametrized as a smooth function of diagonal cut. This is a convincing argument for a smooth background shape. However, to obtain the real QCD background prediction for the 6-jet sample we assume the Landau background shape that works well in the 5-jet sample also describes the background in the 6-jet sample. Since we expect a signal 
around the top mass of $172.5 \mathrm{GeV} / \mathrm{c}^{2}$ we blind the fit to this region. We use the 5 -jet MPV and width as input values for the fit in the 6-jet sample. We also obtain the errors on 5-jet MPV and width and restrict the 6-jet parameters within this range. The overall normalization is allowed to float which is necessary due to different statistics in the two samples. From this new fit we save again the amplitude, MPV and width and plot them versus diagonal cut. All three parameters as a function of diagonal cut for 5-jet (black) and 6-jet (red) can be seen in figure 4.10. As can be seen all values are parametrized as a smooth function of the diagonal cut in the 6-jet as well as in the 5 -jet sample. The difference in the parameters is less than $2 \mathrm{GeV}$ for both samples. We therefore fix the background parametrization from this point to the fits in red in 4.10 when we fit for signal. The 6-jet data mass plots with QCD predictions coming from this parametrization are shown in figure 4.11 .

\subsubsection{All-hadronic $t \bar{t}$ decay}

To estimate the contribution of the all-hadronic $t \bar{t}$ decay in the mass region around 172 $\mathrm{GeV} / c^{2}$ we use Monte Carlo simulation. We incorporate a simulation of the trigger used to select the data into our Monte Carlo sample. We find a diagonal cut of $190 \mathrm{GeV} / \mathrm{c}$ to be the optimal one for the top mass, the procedure for optimizing the diagonal cut for different masses is described in Section 4.4. We estimate the top signal that we expect in $3.2 \mathrm{fb}^{-1}$ of data to be a Gaussian signal including (0.8 \pm 0.3$)$ events and the error includes statistical, theoretical uncertainties in the cross section as well as systematic uncertainties described in Section 5.3. 

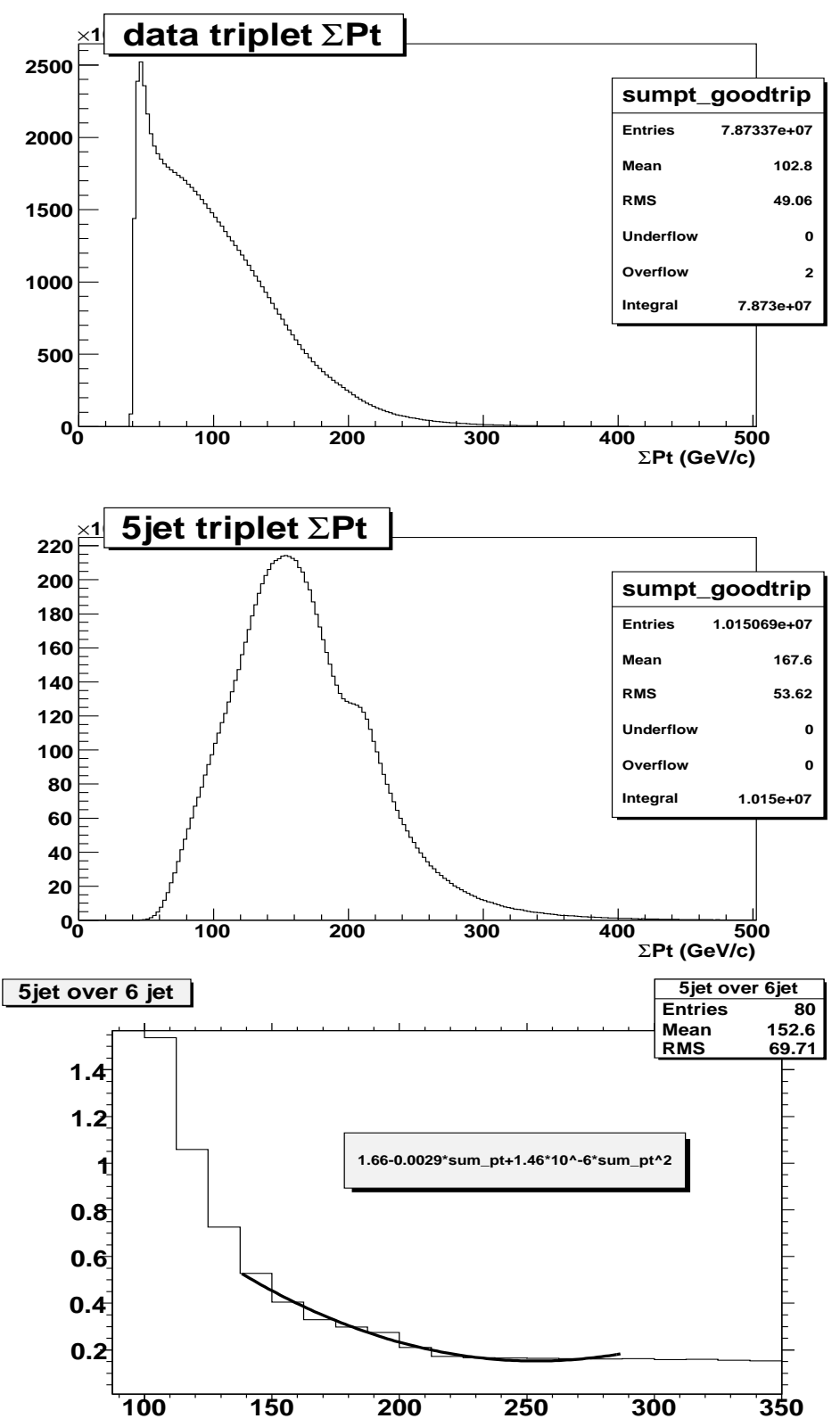

Figure 4.8: $\quad \sum_{j j j}\left|p_{T}\right|$ distributions for 6-jet and 5-jet sample. The first figure above shows the $\geq 6$-jet $\sum_{j j j}\left|p_{T}\right|$ for the data. The second figure shows the $\sum_{j j j}\left|p_{T}\right|$ for the 5 -jet data sample. Finally, the last figure shows the ratio of 5-jet and 6-jet and thus provides a scaling factor for the 5-jet background. Note that the top plot showing the 6-jet sample peaks at low $\sum_{j j j}\left|p_{T}\right|$. This is due to triplets that include the 6 th, 7 th or 8 th jet. Triplets made from the 5-jet sample do not show this feature due to the 4 jet requirement of the trigger. 

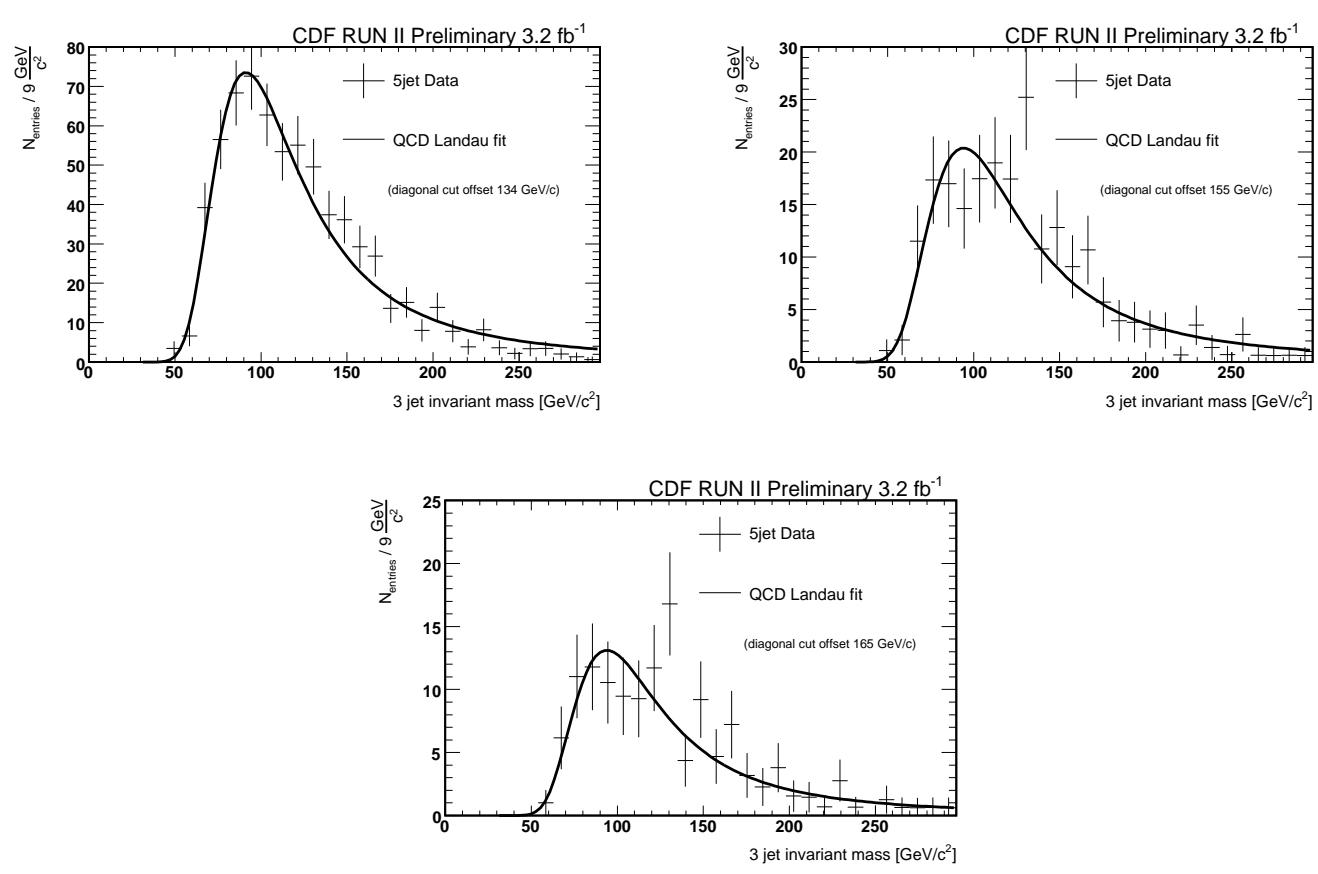

Figure 4.9: 5-jet and 6-jet background. These plots show the 5-jet background shape from the data for different diagonal cuts. The solid line is the fit to a Landau function.

\subsection{Diagonal Cut Optimization}

In this section we describe the method used to find the best diagonal cut for each gluino mass. We first validate the technique by applying it to $t \bar{t}$ in the all-hadronic channel and find its optimal diagonal cut. Afterwards we repeat the procedure for our Monte Carlo gluino samples.

\subsubsection{Pseudoexperiments}

We use a combination of the data-driven QCD background estimate and Monte Carlo simulation to determine the optimal diagonal cut for each gluino mass. We have a parametrization of the background shape for each diagonal cut shown in Figure 4.10. For each set of parameters we create histograms replicating a $M_{j j j}$ background only mass distribution what we call pseudoexperiment. We obtain the signal shape and expected number of events for each diagonal cut from a fit to the $\mathrm{MC}$ signal $M_{j j j}$ 


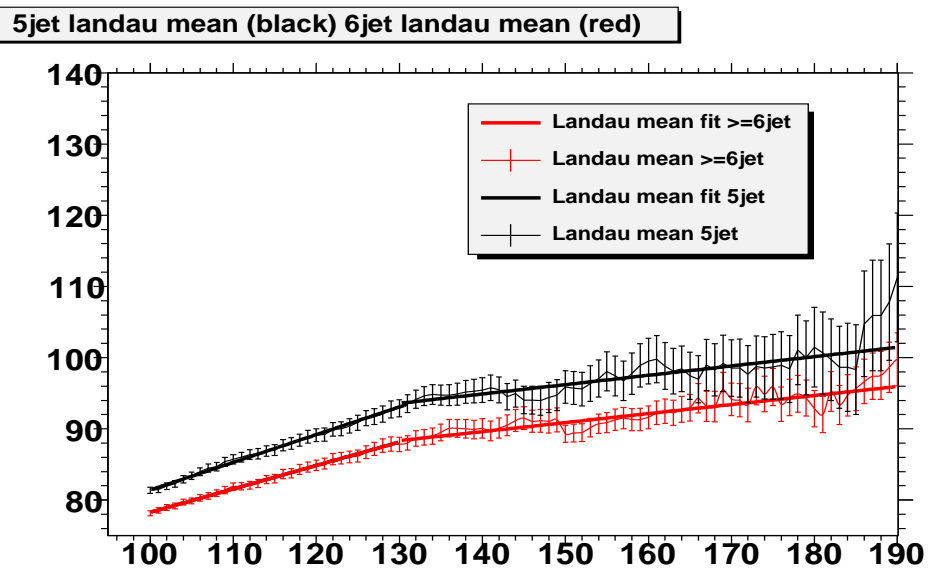

5jet landau width (black) 6jet landau width (red)

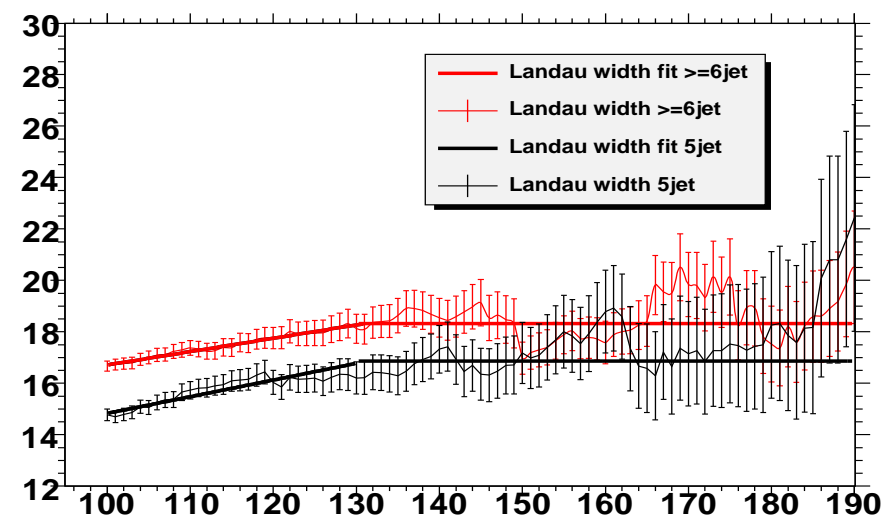

5jet landau amp (black) 6jet landau amp (red)

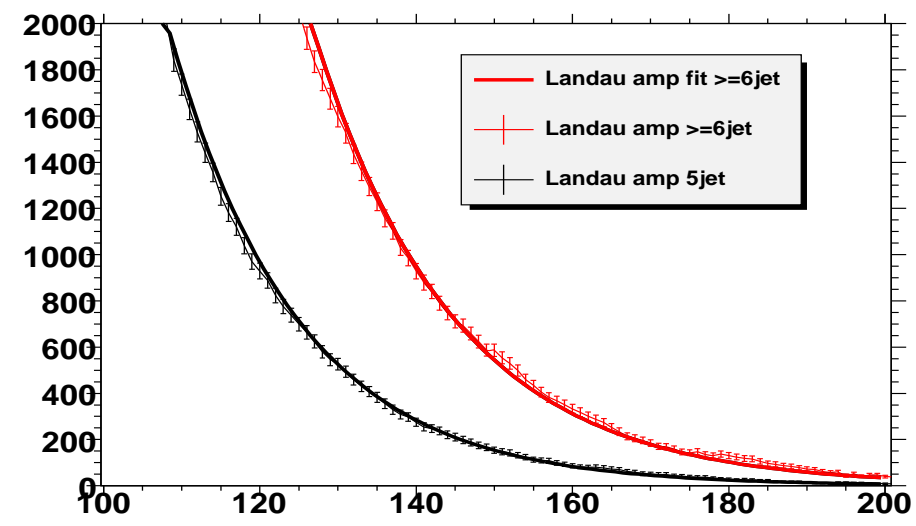

Figure 4.10: Landau parameters for 5-jet and 6-jet background. Landau MPV (top), width (middle) and amplitude (bottom) versus diagonal cut as extracted from the fits. Fits to the scaled 5-jet (black) and 6-jet (red) data are shown. The results of these fits are used in our QCD background estimates. We note that Landau MPV, width and amplitude are fixed to the values given by the red curves when we fit 6-jet data for signal. The difference between the red and the black curves for MPV and width give us an indication of the systematic uncertainty in the background. 

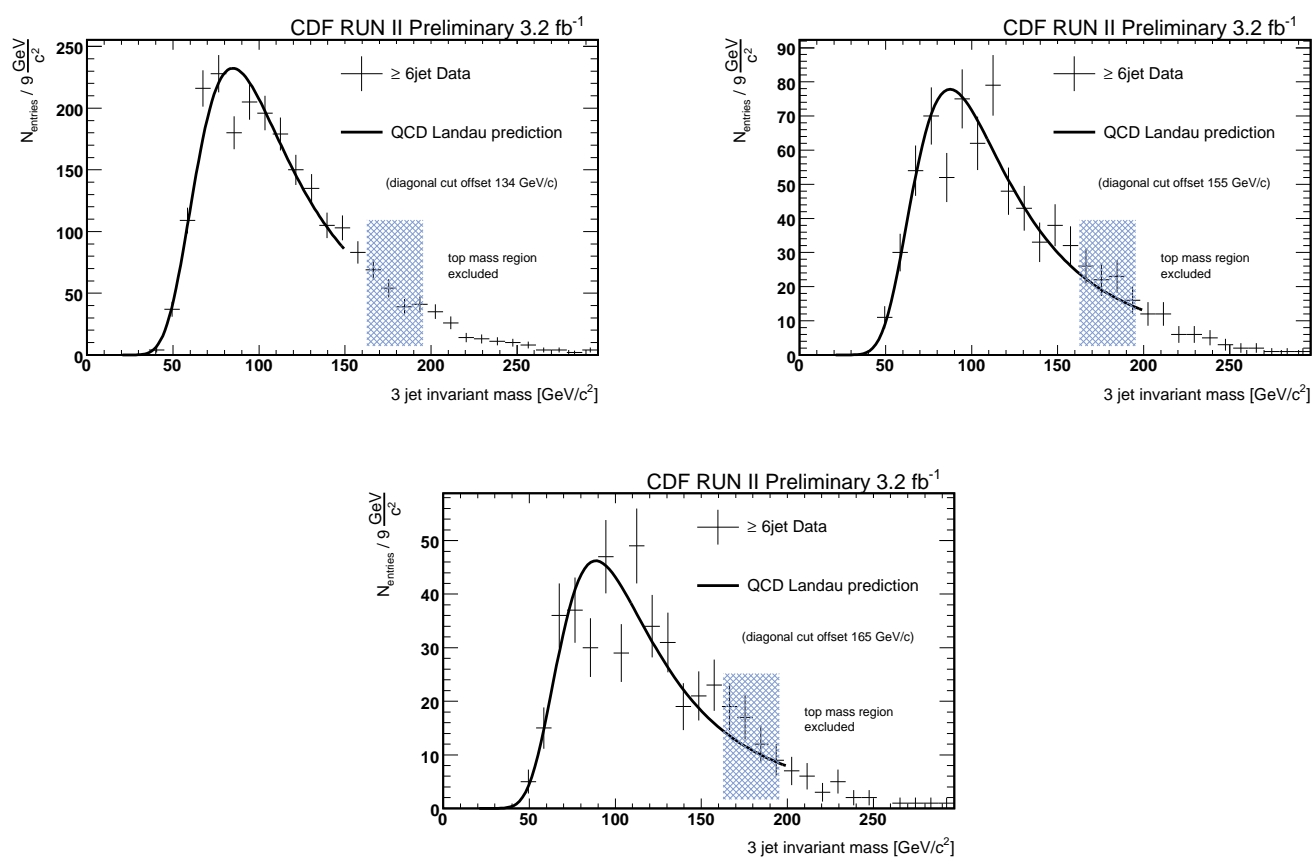

Figure 4.11: These plots show the 6-jet background shape from the data for different diagonal cuts. The solid line is the QCD prediction obtained from the parametrization in 4.10 
distribution. An example is shown in the top left plot in Figure 4.12 for a gluino with a pole mass of $110 \mathrm{GeV} / c^{2}$ for a diagonal cut of $145 \mathrm{GeV} / \mathrm{c}$. The bold line is a fit of a Landau function for the combinatorial background and a Gaussian for the signal. We only count the events in the Gaussian function as signal events. To combine the data QCD background shape and an expected signal we need to rescale the measured events to a luminosity of $3.2 \mathrm{fb}^{-1}$, the size of our dataset. Therefore we use:

$$
N_{\text {evt }}=\sigma \cdot B R \cdot L_{\text {int }} \cdot A,
$$

where $\sigma$ is the cross section, BR stands for the branching ratio, $L_{i n t}$ is the integrated luminosity and A the acceptance. The acceptance for this study is obtained from the integral of the fitted Gauss peak divided by the number of events generated. To not just model number of events but also the shape of the signal we save the width and mean of the Gaussian as well. Using the above mentioned functions for background and signal shape we throw pseudoexperiments according to the sum of background and signal functions. We fit each pseudoexperiment by using a two step fitting procedure. First we blind the signal mass window and fit a Landau distribution for the background shape. After this "pre-fitting", we save the values for MPV, amplitude and width of the Landau as well as the errors of these values. For the final fit which includes a signal Gaussian we allow the Landau shape to float between $\pm 2 \sigma$ of the errors from the prefitting. Since the fitter is extremely sensitive to the initialized values and its limits this pre-fitting should make the fitter well behaved. We define this as one pseudoexperiment and repeat it 1000 times for each diagonal cut. An example for the procedure can be seen in Figure 4.12

\subsubsection{Optimization}

For each pseudoexperiment we obtain the number of signal events and the number of background events. For the number of signal events we integrate the Gaussian in an area of $\pm 1 \sigma$. For the number of background events we integrate the Landau in the same area as the Gaussian. We divide signal by background and save this fraction for each pseudoexperiment and take the mean of 1000 pseudoexperiments. This procedure can 

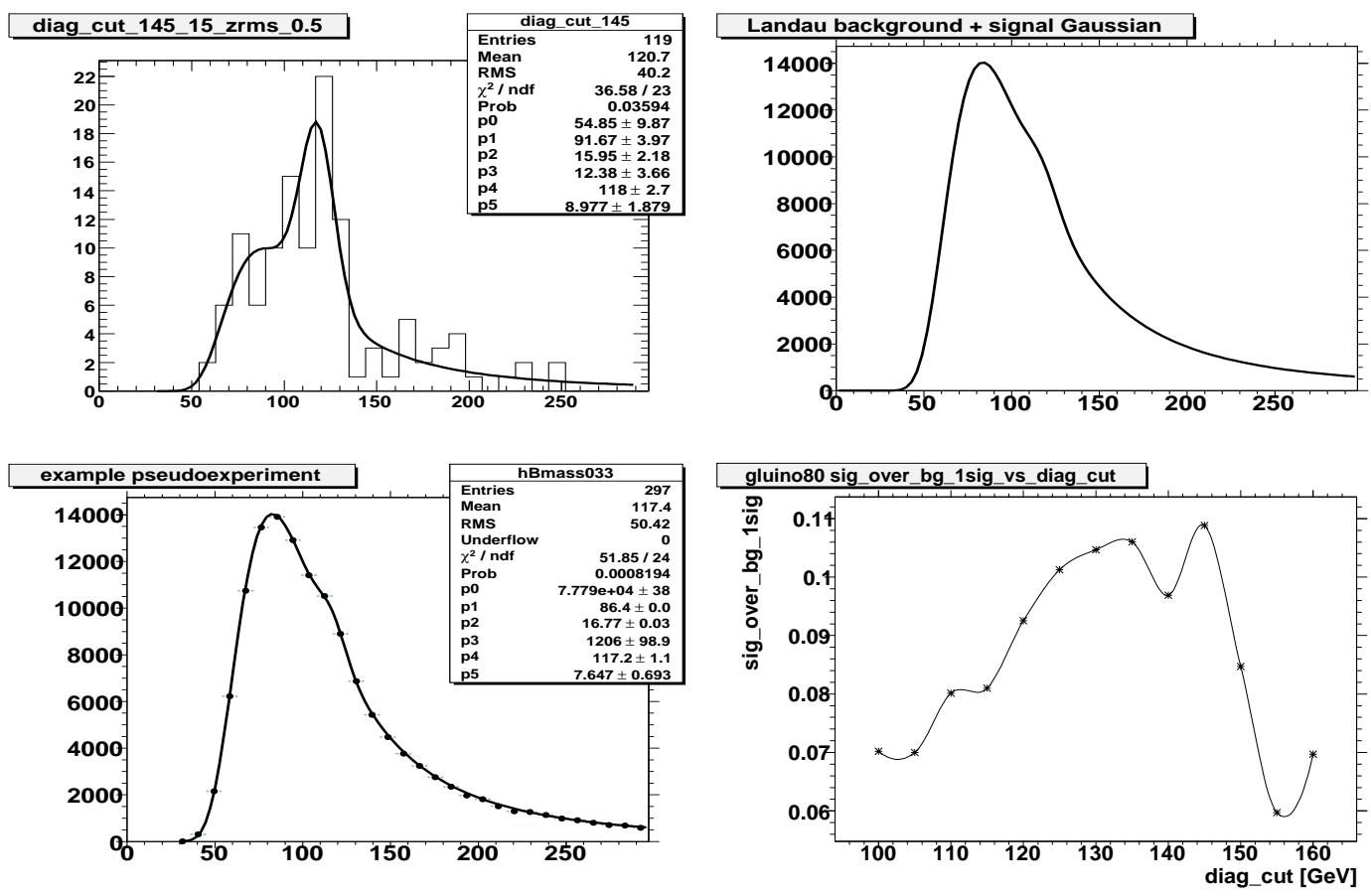

Figure 4.12: Diagonal cut optimization for for a gluino mass of $110 \mathrm{GeV} / c^{2}$. Top left: $M_{j j j}$ distribution for signal MC fitted to a Gaussian and Landau function. Top right: The sum of the signal (Gaussian) and background (Landau) functions from which we throw pseudoexperiments. Bottom left: An example pseudoexperiment. Bottom right: The mean signal over background for 1000 pseudoexperiments vs. diagonal cut. For this mass point, we find $145 \mathrm{GeV} / \mathrm{c}$ to be optimal. 


\begin{tabular}{|c|c|}
\hline pole mass & $\begin{array}{c}\text { optimal } \\
\text { diagonal cut }\end{array}$ \\
\hline 73.7 & 114 \\
110.1 & 145 \\
133.5 & 180 \\
167.9 & 185 \\
190.3 & 195 \\
223.3 & 205 \\
225.0 & 195 \\
\hline
\end{tabular}

Table 4.2: Table for diagonal cut optimization for different gluino pole masses from 74 $\mathrm{GeV} / c^{2}$ to $245 \mathrm{GeV} / c^{2}$.

\begin{tabular}{|c|c|c|c|c|c|}
\hline pole mass & diagonal cut & $N_{\text {sig }}$ & $N_{\text {gen }}$ & acceptance $\left[10^{-5}\right]$ & Stat error $\left[10^{-5}\right]$ \\
\hline 73.73 & 110 & 9.9 & 294258 & 3.9 & 1.2 \\
110.08 & 152 & 10.6 & 266587 & 3.9 & 1.2 \\
133.46 & 180 & 68.2 & 908032 & 7.51 & 0.91 \\
167.87 & 190 & 39.9 & 739046 & 5.40 & 0.26 \\
190.25 & 192 & 41.2 & 813785 & 5.07 & 0.79 \\
223.26 & 196 & 30.1 & 780543 & 3.86 & 0.71 \\
244.95 & 199 & 30.2 & 751794 & 4.02 & 0.73 \\
\hline
\end{tabular}

Table 4.3: Table of gluino acceptances

be repeated for each value of diagonal cut and for each of our gluino samples as well as $t \bar{t}$. Figure 4.13 shows the signal divided by background distribution for the different gluino samples versus diagonal cut. The optimal diagonal cut that we choose for each gluino mass can be seen in Table 4.2. The bottom right plot shows this optimization for the top quark, which is optimal for a diagonal cut of $190 \mathrm{GeV} / \mathrm{c}$.

Now that we have these numbers, we plot them versus the pole mass in Figure 4.14 and fit two linear functions to have an estimate for the diagonal cut between the mass points. Table 4.2 summarizes the optimal value of the diagonal cut for different gluino masses.

\subsection{Acceptance}

To finalize the analysis we obtain acceptances from the gluino Monte Carlo samples. We include the trigger efficiency which is a simulation of the multijet trigger which is 

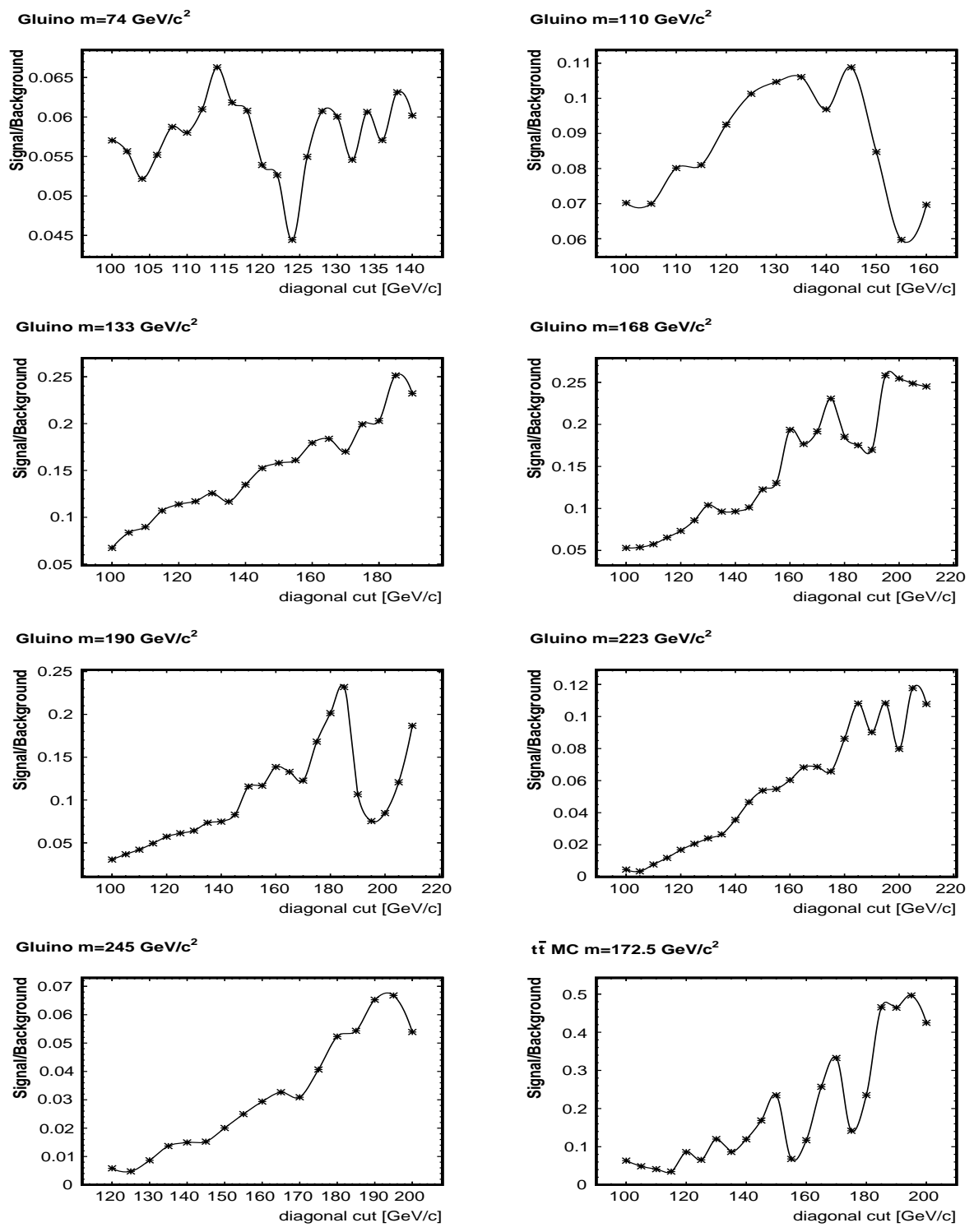

Figure 4.13: Gluino signal/background fraction as a function of diagonal cut. The plots above show the gluino signal versus the diagonal cut for gluino masses from $74 \mathrm{GeV} / c^{2}$ to $245 \mathrm{GeV} / c^{2}$ and the $t \bar{t} \mathrm{MC}$ sample. 


\section{Optimal Diagonal cut}

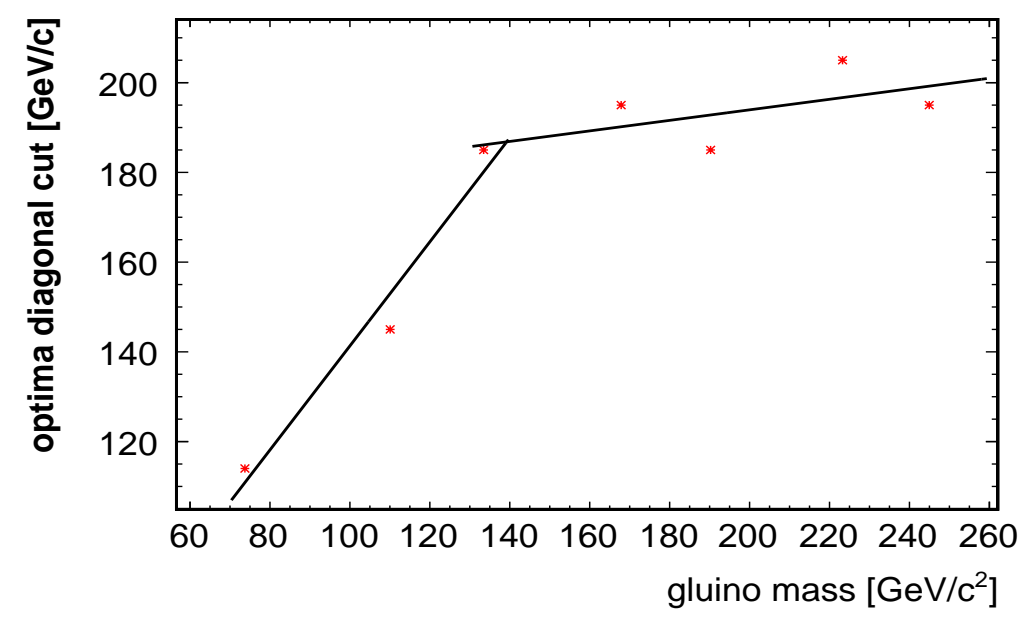

Figure 4.14: Optimal diagonal cut for each gluino mass.

\section{Gluino Acceptance}

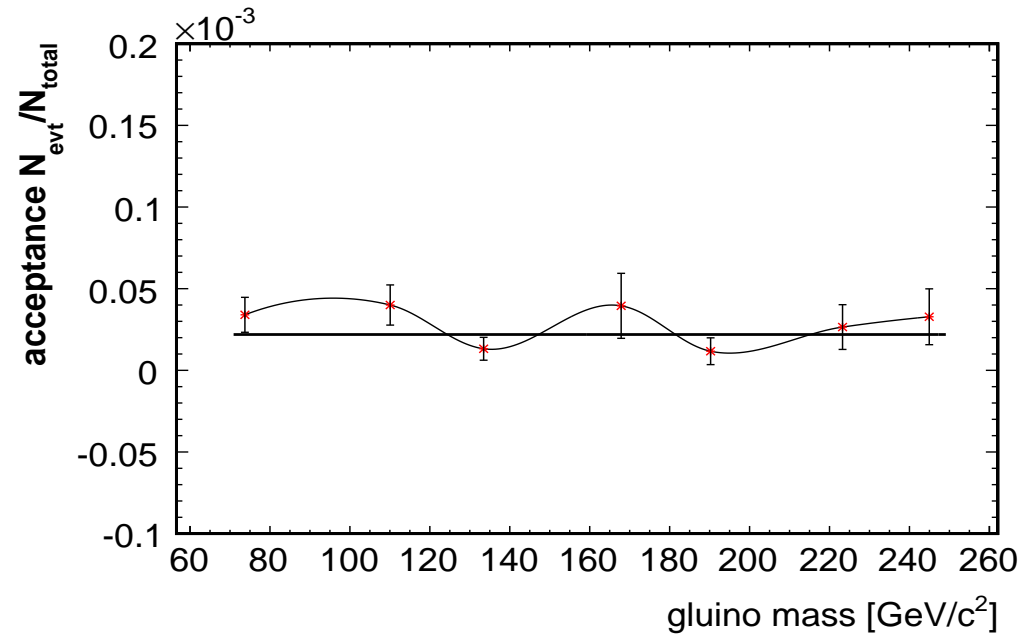

Figure 4.15: Acceptance for each gluino mass from $74 \mathrm{GeV} / c^{2}$ to $245 \mathrm{GeV} / c^{2}$. We note that the acceptance is independent of the mass. 
used for our dataset into the event selection as described in Reference [27]. To compare with the mass point that our scan runs over we use the fit functions from Figure 4.14 and evaluate a diagonal cut for each mass. From the fit to the $M_{j j j}$ distribution we obtain the number of events that pass all the cuts. These are represented by the area of $\pm 1 \sigma$ of the fitted signal Gaussian on top of a Landau function for the combinatorial background. This number is divided by the number of events generated. Table 4.3 shows all the values for the different gluino masses. We find that to within statistical uncertainties, the acceptance is constant as a function of gluino mass, as can be seen in Figure 4.15. We parameterize it as a constant value of $(4.96 \pm 1.1) \cdot 10^{-5}$ including the statistical error. 


\section{Chapter 5}

\section{Setting Limits on Hadronic Resonances}

We do not observe an excess in the data and place limits on the cross section for $\sigma(p \bar{p} \rightarrow \tilde{g} \tilde{g} \rightarrow 3 j e t s+3 j e t s)$. The following chapter describes the statistical methods used to set this limit as well as a description of systematic uncertainties. The cross section as a function of gluino mass is presented at a $95 \%$ confidence limit.

\subsection{Bayesian approach for observed $95 \%$ confidence limit calculation}

In order to calculate the number of observed events at a $95 \%$ confidence limit we use a bayesian approach. Therefore we need the background estimate, which is given by a Landau function and the fixed parameters as described in Section 4.3. Since we model these parameters as a function of diagonal cut we use the optimal cut for each mass as described in Section 4.4. We scan through the mass range of $76.5 \mathrm{GeV} / c^{2}$ to 238.5 $\mathrm{GeV} / c^{2}$, in steps of $9 \mathrm{GeV} / c^{2}$ which corresponds to the middle of each bin. For each mass point we fix the Landau background parameters according to the fit functions from Section 4.3 and try to fit a Gaussian signal shape on top of that. We restrict the Gaussian to have a width between 7 and $15 \mathrm{GeV} / c^{2}$, since this is the typical width of a resonance we would expect from the gluino Monte Carlo samples. The mass at which we want to scan is fixed as well but the Gaussian amplitude is allowed to float without restrictions. To calculate the limit we define the number of observed events as

$$
n_{i}=S_{i}+B_{i}
$$

where $S_{i}$ is given by the integral of $\pm 1 \sigma$ of the Gaussian fit in the $i^{t h}$ bin. $B_{i}$ is the integral of the background Landau distribution in the same range of the $i^{t h}$ bin. Given this number of background events we calculate a 95\% C.L. by using a Poisson 

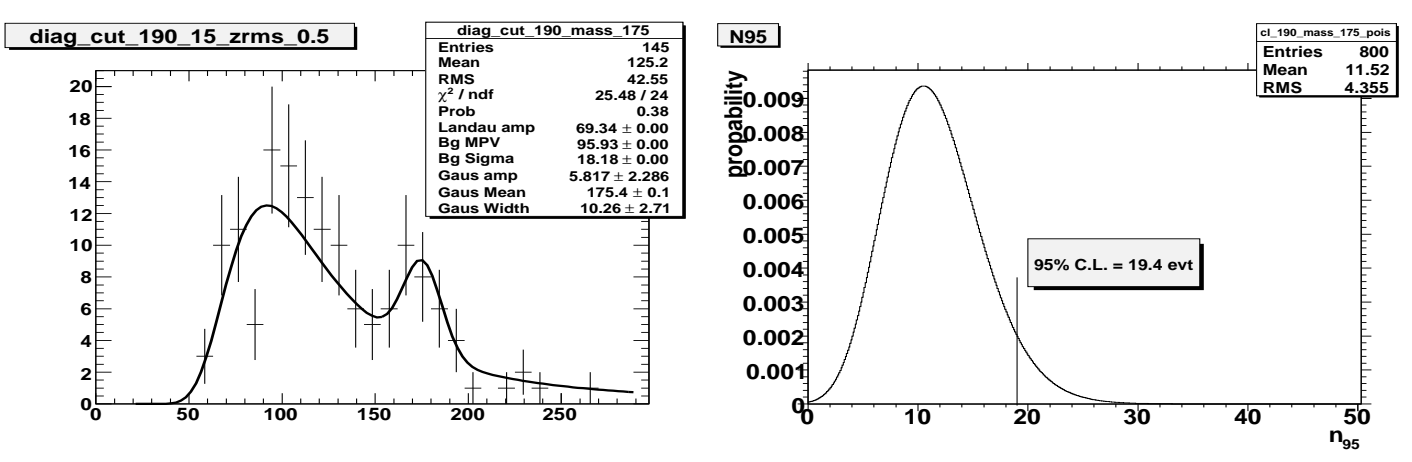

Figure 5.1: On the left side is a fit of Landau background and Gauss signal. The right plot shows the probability density function given 7.6 background and 11.1 signal events.

distribution

$$
P_{i}\left(n_{i}, \mu_{i}\right)=\frac{\mu_{i}^{n_{i}} e^{-n_{i}}}{n_{i} !},
$$

where $\mu_{i}$ is the number of expected events in the $i^{\text {th }}$ bin. This value consists of $\mu_{i}=B_{i}+n_{95}$. Figure 5.1 shows the procedure. We increase $n_{95}$ slowly and fill a histogram with the probabilities. After normalizing the distribution we integrate an area of $95 \%$. In Figure 5.1 the right plot shows a vertical line where we reach this limit. The associated value on the $\mathrm{x}$ axis represents our $95 \%$ confidence limit. We choose a different method using pseudoexperiments to calculate the number of expected events. Table 5.1 summarizes the $95 \%$ upper C.L. on the observed events.

\section{$5.2 \quad$ Expected $95 \%$ confidence limit}

In order to determine how likely it is that our background fluctuates and therefore produces a signal we use pseudoexperiments. We use background pseudoexperiments which are parameterized by a Landau function with parameters from Section 4.3 . In addition we include a Gaussian shape signal at $\mathrm{m}=172 \mathrm{GeV} / c^{2}$ which consists of $0.8 \pm 0.3$ events to account for the contribution of $t \bar{t}$ events in the all-hadronic channel. A more detailed description of the studies with $t \bar{t}$ Monte Carlo and a comparison to data can be seen in Appendix A. We throw 1000 pseudoexperiments with this shape and fit afterwards with the fixed Landau distribution that we started with. In addition to this Landau we try to fit a Gaussian on top of it to measure the background induced signal. 


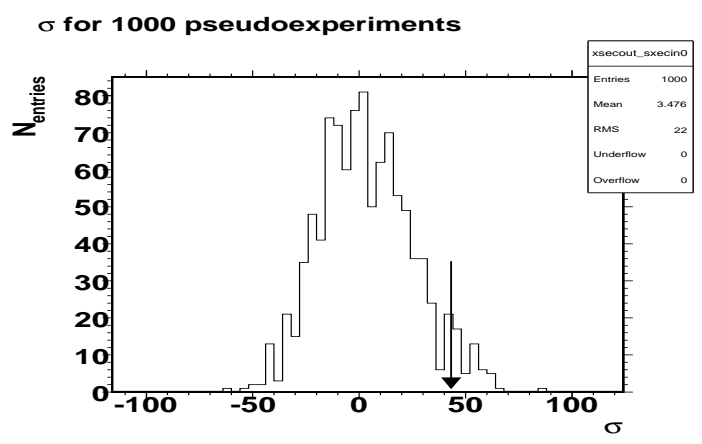

Figure 5.2: Measured cross section distribution for 1000 pseudoexperiments at a mass of $158 \mathrm{GeV} / c^{2}$ without systematic uncertainties.

We save the number of events in a $\pm 1 \sigma$ range of this Gaussian and fill a histogram. After the 1000 pseudoexperiments we find the value of signal events that corresponds to the 95 $\%$ limit. To obtain a cross section we divide this number by luminosity and acceptance. An example of the cross section $\sigma$ and the $95 \%$ C.L. for a mass of $158 \mathrm{GeV} / c^{2}$ can be seen in figure 5.2. To obtain a statistical error band we add a Gaussian shape signal with the cross section corresponding to the $95 \%$ C.L. (referred to as $\sigma^{95 \%}$ ) on top of our background parameterization and throw 1000 pseudoexperiments. In these 1000 pseudoexperiments we measure the input cross section and obtain a statistical error on $\sigma^{95 \%}$ at $68 \%$ and $95 \%$ which correspond to $\pm 1 \sigma$ and $\pm 2 \sigma$ error band on the expected 95\% C.L. limit.

\subsection{Systematic uncertainties}

We divide the systematic uncertainties into two broad categories: uncertainties in the shape of the $M_{j j j}$ distribution, background shape systematics, and uncertainties in the acceptance of the signal.

\subsubsection{Linearity check}

We perform a cross check to ensure that our pseudoexperiments function properly. Therefore we add a signal with a given cross section and a Gaussian shape on top our Landau QCD background prediction. We now throw 1000 pseudoexperiments with 


\begin{tabular}{|c|c|c|c|}
\hline mass & $N_{b g}$ & $N_{\text {sig }}$ & $95 \%$ C.L. \\
\hline 76.5 & 1161.2 & -58.9 & 41 \\
85.5 & 601.2 & -73.0 & 22.5 \\
94.5 & 409.8 & -40.4 & 23.5 \\
103.5 & 185.7 & 6.8 & 35 \\
112.5 & 93.3 & 13.5 & 34 \\
121.5 & 54.7 & 10.3 & 27 \\
130.5 & 23.7 & 3.7 & 14.3 \\
139.5 & 12.2 & -0.43 & 7.8 \\
148.5 & 21.1 & 7.6 & 17.5 \\
157.5 & 17.0 & 11.6 & 21.4 \\
166.5 & 10.8 & 12.4 & 21.8 \\
175.5 & 7.6 & 11.1 & 19.2 \\
184.5 & 6.6 & 8.2 & 15.3 \\
193.5 & 7.5 & 5.2 & 12.1 \\
202.5 & 2.9 & -0.9 & 3.7 \\
211.5 & 2.4 & -2.0 & 3 \\
220.5 & 3.4 & -0.7 & 4.4 \\
229.5 & 1.7 & 0.6 & 5 \\
238.5 & 1.3 & 0.06 & 4 \\
\hline
\end{tabular}

Table 5.1: Number of observed events at $95 \%$ C.L.

Table shows mass, fitted number of background events, number of observed signal events and $95 \%$ C.L. 


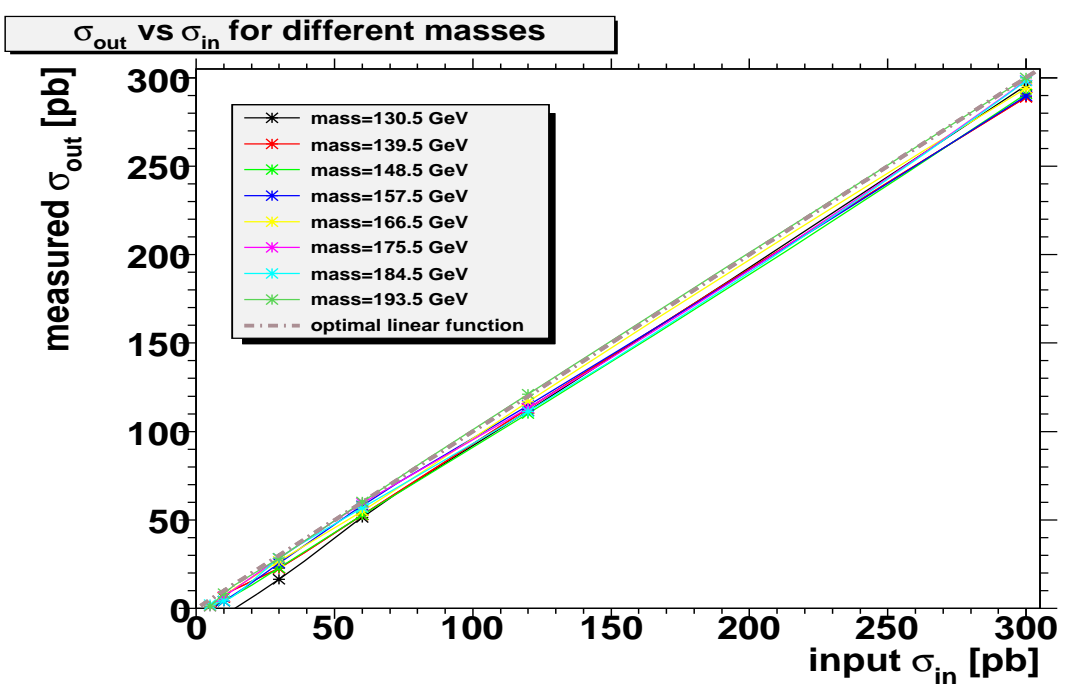

Figure 5.3: Input cross section vs. measured cross section for varying masses.

this setup for different input cross sections and different mass points. We measure the output cross section as a function of the input cross section which is shown in Figure 5.3 Each point represents the median of 1000 pseudoexperiments. We see a linear dependence which is independent of the mass.

\subsubsection{Background shape systematics}

Even though we use a fixed parametrization for the QCD background shape this technique is not without an error. In Figure 4.10 we show the parameterization for the background Landau function. We clearly see a small difference between the weighted 5-jet and 6-jet sample as well as error bars on the MPV and width of the Landau function. In order to incorporate the uncertainties in our background estimate into the final limit plot we add a randomness to Landau MPV, width and amplitude when we throw pseudoexperiments. For each pseudoexperiment we pick values for MPV, width and amplitude in a percentage range around the nominal value which is different for each pseudoexperiment. Below a diagonal cut of $130 \mathrm{GeV} / \mathrm{c}$ this means a $1 \%$ range of the amplitude and $5 \%$ range in width and mean, above this diagonal cut we pick $1 \%$ around the amplitude and $10 \%$ for width and mean. With this set of parameters we 


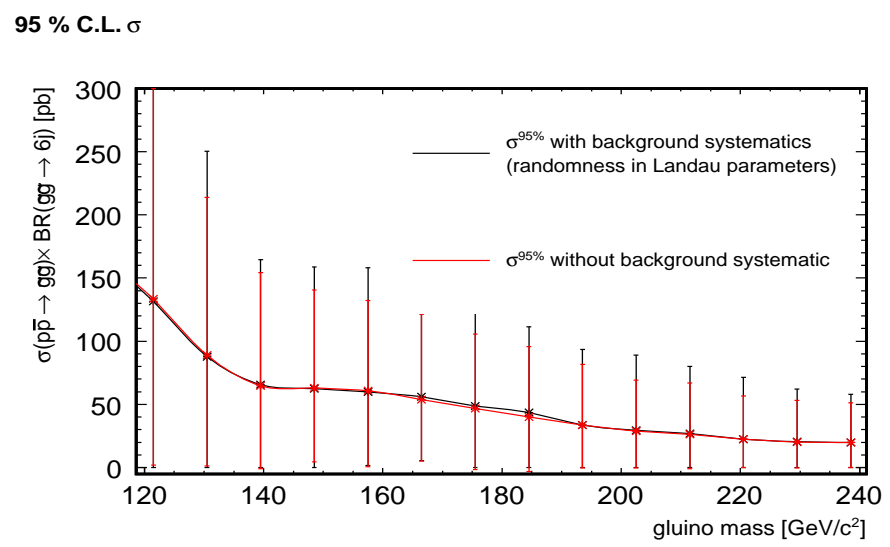

Figure 5.4: Cross section limit plot with background systematic. The error band is obtained by using the described randomness in the Landau background parameters. The acceptance systematic uncertainty is not applied.

produce 1000 different pseudoexperiments and we try to fit a Landau+Gaussian. For the Landau fit we keep the parameters fixed to the original nominal values from figure 4.10 and only the Gaussian is allowed to float. The effect of adding this randomness to the parameters can be seen in figure 5.4. As an example we show the effect when we include a Gaussian signal corresponding to the $95 \%$ C.L. limit and measure the output cross section with its statistical error band. It makes the cross section distribution wider compared to pseudoexperiments thrown without randomness in the parameters. This leads to an increase of the nominal $95 \%$ as well as a widening of the statistical error band. 


\section{Cross check: Overall shift of Landau parameters}

We also perform a different study to see how an overall shifting of the background parameters would affect the final limit plot. Figure 5.6 shows in yellow the mean between 5jet and 6jet parameterization. We build errors symmetric around this value.

To study the influence of the parameterization further we repeat the pseudoexperiments as described in Section 5.2 by moving the parameterization within the errors assigned in figure 5.6. When we move the parameterization "up", this refers to increasing the amplitude by $1 \%$ and picking the upper errors for the mean and width. This will make the distribution wider and moves the mean to a higher value. When we move it "down", we decrease the amplitude by $1 \%$ and make the distribution narrower as well as shift the mean to a lower value. We overlay the limit plots for the three different parametrizations, up, down and nominal, which is shown in the figure 5.7 on the left. As described before the up value increases the overall normalization as well as the position of the background, therefore with a higher number of background events the fitted signal is more likely to fluctuate up. Reducing the background therefore leads a lower fitted signal. This behavior can be seen in figure 5.7 where we see a wider and narrower $\pm 2 \sigma$ error band. 


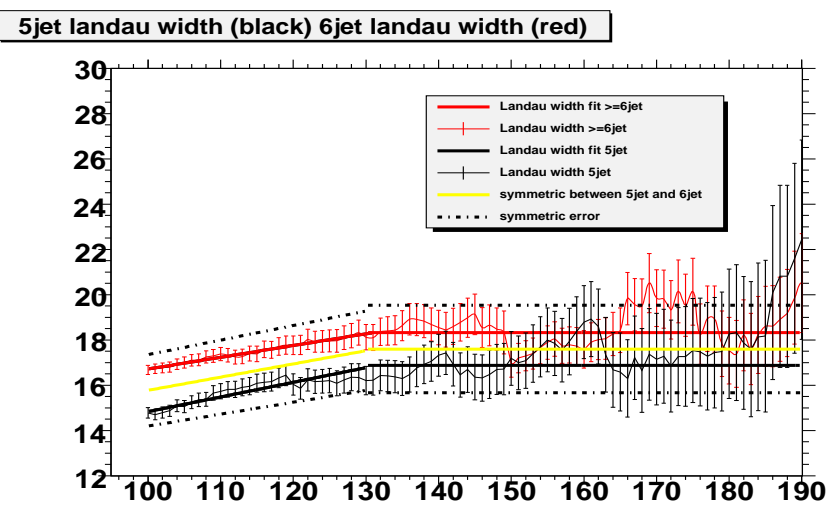

Figure 5.5: Landau width with symmetric errors around the mean of 5jet and 6 jet.

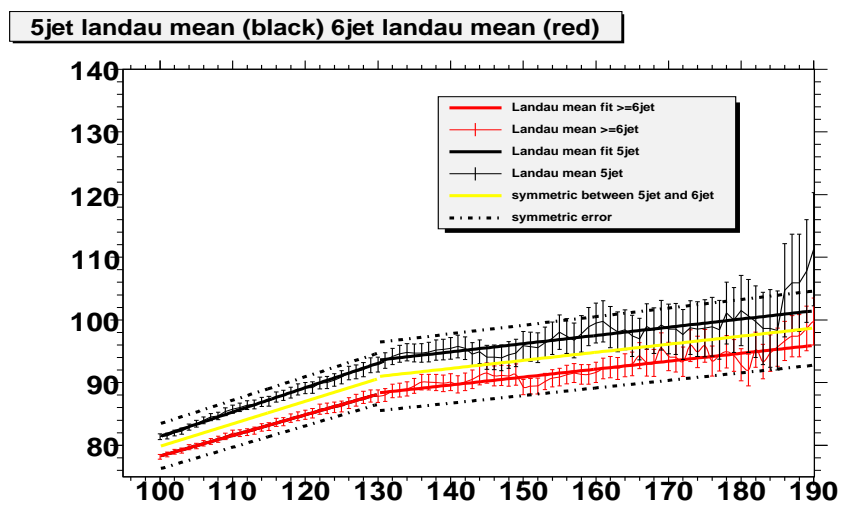

Figure 5.6: Landau mean with symmetric errors around the mean of 5jet and 6jet. $95 \%$ C.L. $\sigma$

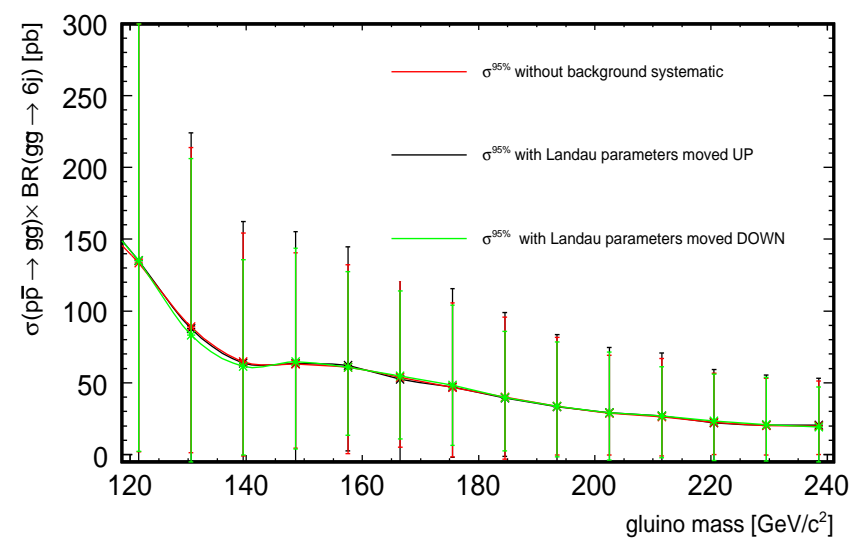

Figure 5.7: Cross section limit plot with over all shift in background parameters. The acceptance systematic uncertainty is not applied. 

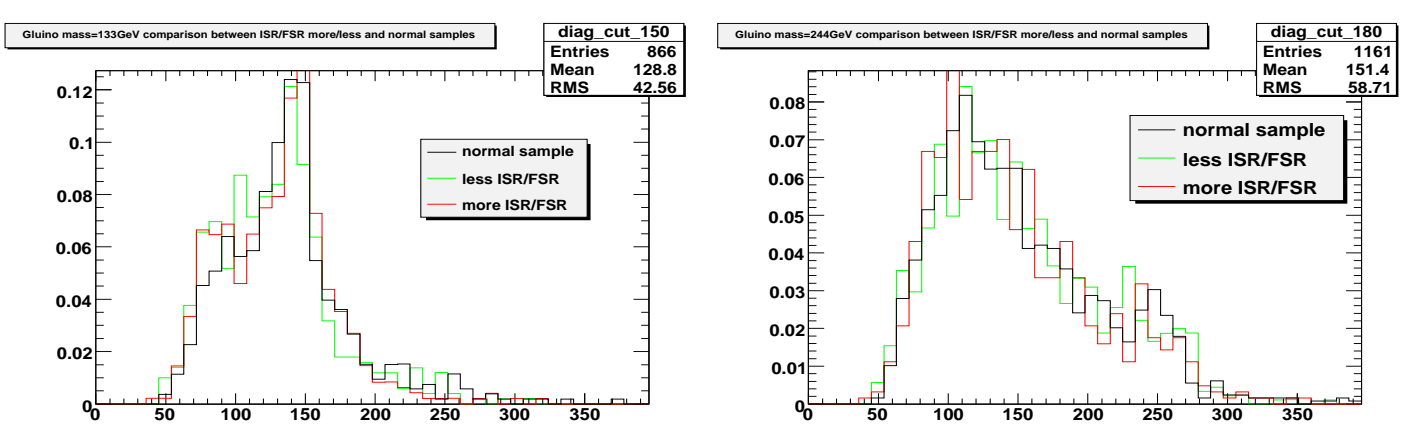

Figure 5.8: Comparison of more/less ISR/FSR. Left: ISR/FSR less, normal, more for gluino $\mathrm{m}=133 \mathrm{GeV} / c^{2}$. Right: ISR/FSR less, normal, more for gluino $\mathrm{m}=244 \mathrm{GeV} / c^{2}$.

\subsubsection{ISR/FSR}

To estimate the effect of initial (ISR) and final state radiation (FSR) on our acceptance we generate two gluino samples with pole mass of $\mathrm{m}=133.1 \mathrm{GeV} / c^{2}$ and $\mathrm{m}=244.5$ $\mathrm{GeV} / c^{2}$, with more or less ISR/FSR. For an estimate for a mass point between these two masses we use the $t \bar{t}$ Monte Carlo sample with more/less ISR and FSR where the effect in the $t \bar{t}$ acceptance and therefore in the number of expected events can be seen in table A.1. Figure 5.8 shows a comparison of the nominal gluino samples to the ISR/FSR more or less samples. Since the shape of the signal itself does not change much we estimate an overall error of $20 \%$ for this systematic by comparing number of events that pass all cuts in a mass window around the pole mass.

\subsubsection{Jet Energy Scale}

The effect of the different Jet Energy Scales are described in Section 4.1.1. These correction are not free of uncertainties. To account for these uncertainties we apply a $\pm 1 \sigma$ JES shift to all of our gluino samples. This yields to the results summarized in Table 5.2, which compares the up and down shift with the original acceptance as a function of gluino mass [38]. We assign an overall error of $31 \%$ for this systematic. 


\begin{tabular}{|c|c|c|}
\hline pole mass & JES $+1 \sigma[\%]$ & JES $-1 \sigma[\%]$ \\
\hline 133.4 & 18.4 & 25.3 \\
167.8 & 21.4 & 25.5 \\
190.5 & 18.6 & 33.8 \\
223.2 & 19.5 & 22.6 \\
244.9 & 20.9 & 46.3 \\
\hline
\end{tabular}

Table 5.2: Effect of the jet energy scale on gluino acceptance. Table shows pole mass and percentage change in acceptance after applying a $\pm 1 \sigma$ JES

\subsubsection{PDF}

We use a special tool to evaluate the effect of varying Parton Distribution Functions (PDF) on the RPV gluino acceptance. The utility reads in various sets of PDF's and calculates $Q^{2}$ and $x$ for each event, and determines an event-weight corresponding to each PDF set. The PDF sets investigated are MRST75, as well as various CTEQ6 sets. We compare these to the nominal (CTEQ5) PDF that was used to generate the events. We find the largest difference in acceptance is between the CTEQ6M and CTEQ5 sets. We find that the imposition of the diagonal cut causes the biggest PDF-related change in acceptance. This is understandable, since the diagonal cut isolates high-boost final states, which are most sensitive to changes in PDF. We find that the acceptance changes from $4 \%$ for low-mass $\left(100 \mathrm{GeV} / c^{2}\right)$, low diagonal cut samples, to $10 \%$ for high-mass $\left(200 \mathrm{GeV} / c^{2}\right)$, high diagonal cut samples. We assign an overall acceptance systematic uncertainty of $10 \%$ due to PDF's.

\subsubsection{Luminosity}

Another source for systematic uncertainties is the luminosity measurement. CDF uses Cherenkov Luminosity Counters (CLC) to measure the luminosity of the Tevatron [41]. An overall uncertainty of $6 \%$ is assigned. 


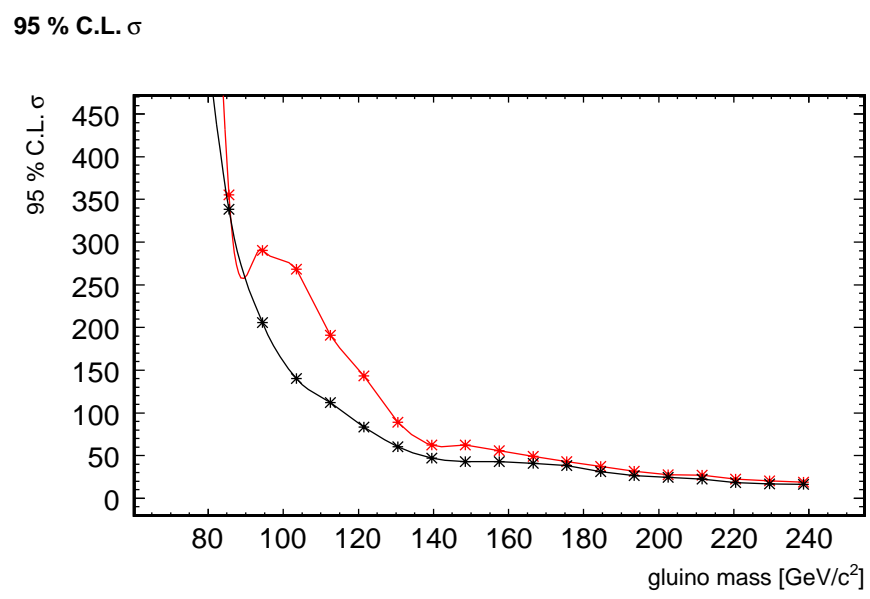

Figure 5.9: black: without systematics, red: with systematics.

\subsubsection{Summary of systematics}

After applying all the above mentioned shifts separately and adding all the relative errors in quadrature and obtain an overall uncertainty of $38 \%$. We include the acceptance uncertainty into our pseudoexperiments. This has the same effect as the uncertainty in the background shape it makes the cross section distribution wider and therefore the 95\% C.L. gets shifted up. Figure 5.9 shows a comparison of the final cross section limit without systematics (black) and with systematics (red). We include $0.8 \pm 0.3$ expected top events into our background around a mass of $172.5 \mathrm{GeV} / c^{2}$. 


\section{Chapter 6}

\section{Results}

We have presented a first search for a new hadronic resonance in $3.2 f^{-1}$ of $p \bar{p}$ collisions at $\sqrt{s}=1.96 \mathrm{TeV}$ using the $\mathrm{CDF}$ II detector. We find no significant excess in the data beyond the known Standard Model processes. We introduced a new technique to extract multijet resonances from QCD background. The presented jet ensemble technique takes advantage of kinematic quantities and their correlations. By selecting high $\sum\left|p_{T}\right|$ objects we are also accessing an interesting region of phase space. The search is model independent and could be performed for any pair produced hadronic resonance decaying into three jets. We choose a R-parity violating pair produced gluino as a possible new physics scenario and place a 95\% C.L. upper limit in the production cross section $\sigma(p \bar{p} \rightarrow \tilde{g} \tilde{g} \rightarrow 3 j e t s+3 j e t s)$ as a function of gluino mass. The systematic uncertainties are incorporated into pseudoexperiments to determine an expected cross section for mass points in a range of $76.5 \mathrm{GeV} / c^{2}-238.5 \mathrm{GeV} / c^{2}$. Figure 6.1 shows the 95\% C.L. upper limits of the expected (black) and observed (red points) cross section on a linear as well as a logarithmic scale. The error band represent the $\pm 1 \sigma$ and $\pm 2 \sigma$ statistical uncertainties. The top and the bottom plot in Figure 6.1 shows the limit plot including a dashed line for the leading order theoretical cross section for pair produced RPV gluinos calculated by PYTHIA multiplied by a k-factor to account for next to leading order effects. We can exclude gluinos with a mass below $144 \mathrm{GeV} / c^{2}$. Table 6.1 shows the values of mass and corresponding expected 95\% C.L. limit as well as the observed $95 \%$ C.L. limit. We note a $2 \sigma$ excess around the top mass. Further studies addressing this excess are described in Appendix $\mathrm{A}$ 

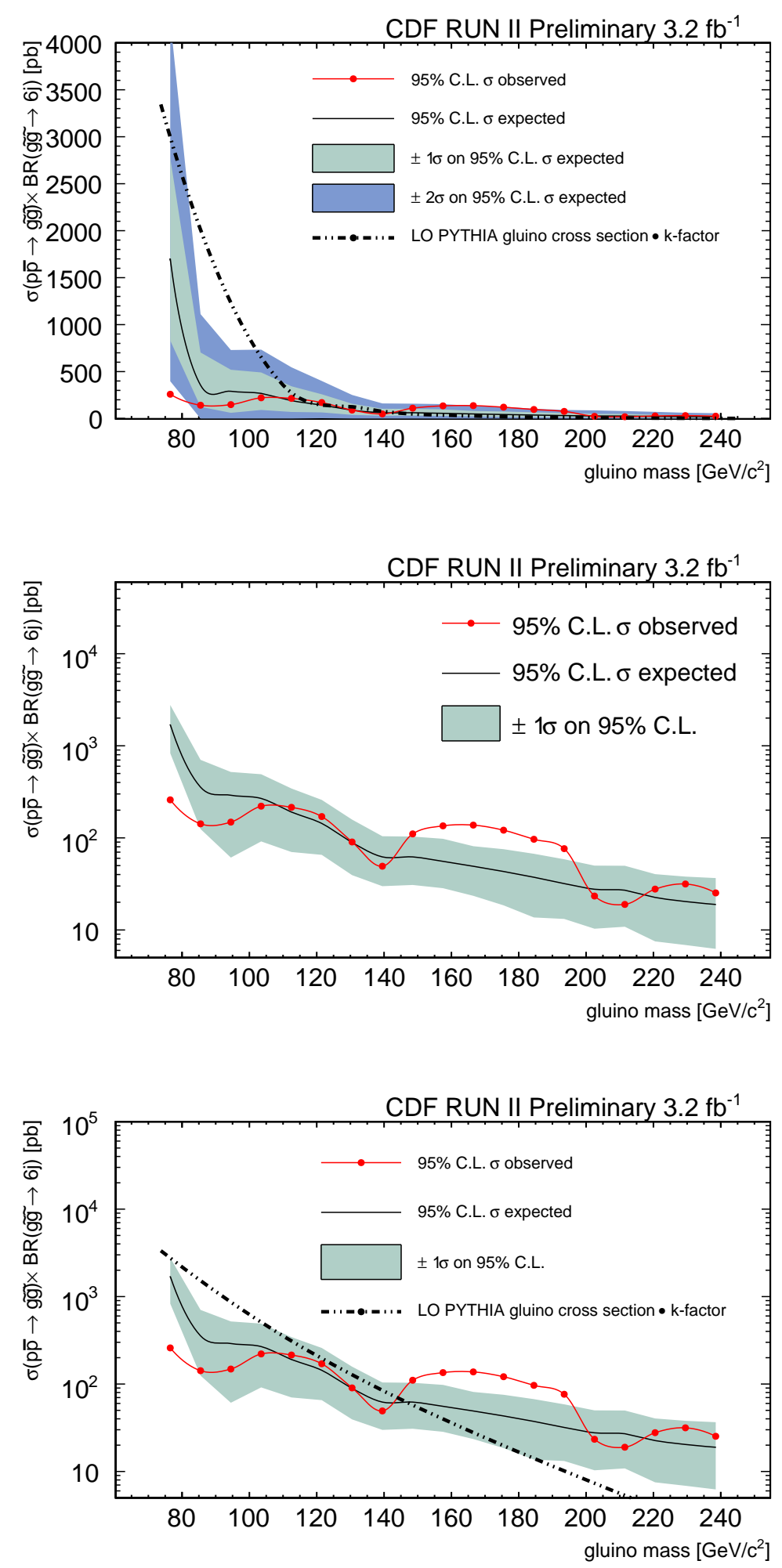

Figure 6.1: The 95\% C.L. upper limit on the expected (black) and observed (red points) cross section. The bands represent $\pm 1 \sigma$ and $\pm 2 \sigma$ uncertainties on this limit. Top: linear scale, Middle: logarithmic scale, Bottom: logarithmic scale. The top and bottom plot include the leading order theory cross section multiplied by k-factor to account for next to leading order effects represented by the dashed line. 


\begin{tabular}{|c|c|c|}
\hline $\begin{array}{c}\text { mass } \\
\mathrm{GeV} / c^{2}\end{array}$ & $\begin{array}{c}\text { expected 95\% C.L. limit. } \\
\mathrm{pb}\end{array}$ & $\begin{array}{c}\text { observed } 95 \% \text { C.L. limit. } \\
\mathrm{pb}\end{array}$ \\
\hline 76.5 & 1704 & 258.3 \\
85.5 & 355.3 & 141.8 \\
94.5 & 292.3 & 148.1 \\
103.5 & 269.8 & 220.5 \\
112.5 & 194.7 & 214.2 \\
121.5 & 143.76 & 170.1 \\
130.5 & 89.7 & 90.1 \\
139.5 & 64.3 & 49.1 \\
148.5 & 62.7 & 110.3 \\
157.5 & 56.25 & 134.8 \\
166.5 & 50.02 & 137.3 \\
175.5 & 43.79 & 121.0 \\
184.5 & 37.9 & 96.4 \\
193.5 & 32.4 & 76.2 \\
202.5 & 28.2 & 23.3 \\
211.5 & 27.52 & 18.9 \\
220.5 & 23 & 27.7 \\
229.5 & 20.9 & 31.5 \\
238.5 & 19.9 & 25.2 \\
\hline
\end{tabular}

Table 6.1: Table shows expected $95 \%$ C.L. limit as well as observed $95 \%$ C.L. limit for mass points between $76.5 \mathrm{GeV} / \mathrm{c}^{2}$ and $238.5 \mathrm{GeV} / \mathrm{c}^{2}$ in $9 \mathrm{GeV} / \mathrm{c}^{2}$ steps. 


\section{Appendix A Studies for the Top quark}

We note that the limit plot in figure 6.1 shows a $2 \sigma$ excess around the top mass. From Monte Carlo prediction we only expect to see $0.8 \pm 0.3$ events in our used data sample of $3.2 \mathrm{fb}^{-1}$. When we fit a signal at a mass of $175 \mathrm{GeV} / \mathrm{c}^{2}$ we obtain $11 \pm 5$ observed events, including the error on the fit parameters. In this appendix we present ideas to further distinguish possible signal from QCD background using jet ensembles. Most example plots are shown as a Monte Carlo study. Performing the analysis in the other decay channels of the top quark, in particular the semi-leptonic decay, could also be considered. However, a full analysis of all the presented ideas would go to far for this thesis and can be found in Reference [42].

\section{A.1 Different Monte Carlo Samples}

We study the influence of different Monte Carlo generators including next to leading order effects as well as initial and final state radiation ISR/FSR. We use a diagonal cut of $190 \mathrm{GeV} / \mathrm{c}$ for all MC samples and fit a Gaussian on top of a Landau function for the combinatorial background and evaluate the Gaussian integral in a $\pm 1 \sigma$ range. The expected events rescaled to $3.2 \mathrm{fb}^{-1}$ for different $\mathrm{MC}$ samples can be seen in Table A.1. These values do not include the multijet trigger simulation which reduces the number of events by $\approx 60 \%$.

\section{A.2 Dalitz plots}

A useful tool to distinguish between QCD background and signal are so-called Dalitz plots. This analysis technique was first used by R.H. Dalitz in his paper in 1954 on the $\tau$ meson (today $\mathrm{K}$ meson) decay into 3 pions. [43]. To define the Dalitz variables we 


\begin{tabular}{|c|c|c|c|}
\hline $\begin{array}{c}\text { Description } \\
\text { PYTHIA }\end{array}$ & $M_{\text {top }}$ & Cross Section & Expected Evt \\
& 172.5 & 7.5 & $1.80 \pm 0.08_{-0.22}^{+0.12}$ \\
\hline PYTHIA & 175 & 7 & $1.42 \pm 0.07_{-0.18}^{+0.08}$ \\
\hline PYTHIA, High Luminosity & 175 & 7 & $0.97 \pm 0.15_{-0.12}^{+0.06}$ \\
\hline PYTHIA, ISR/FSR more & 175 & 7 & $1.48 \pm 0.13_{-0.19}^{+0.08}$ \\
PYTHIA, ISR/FSR less & 175 & 7 & $1.53 \pm 0.13_{-0.20}^{+0.09}$ \\
\hline PYTHIA, MRST72 & 175 & 7 & $1.29 \pm 0.16_{-0.26}^{+0.12}$ \\
PYTHIA, MRST75 & 175 & 7 & $1.48 \pm 0.17_{-0.28}^{+0.13}$ \\
\hline PYTHIA, ISR/FSR more & 172.5 & 7.5 & $1.81 \pm 0.12_{-0.22}^{+0.12}$ \\
PYTHIA, ISR/FSR less & 172.5 & 7.5 & $1.67 \pm 0.12_{-0.20}^{+0.11}$ \\
\hline ALPGEN+PYTHIA & 172.5 & $3.87,1.21$ & $1.57 \pm 0.11_{-0.19}^{+0.10}$ \\
\hline MC@NLO CTEQ5M & 175 & 7 & $1.26 \pm 0.18_{-0.17}^{+0.07}$ \\
\hline MC@NLO MRST75 & 175 & 7 & $0.79 \pm 0.16_{-0.12}^{+0.06}$ \\
\hline \hline
\end{tabular}

Table A.1: Expected top events from different MC samples. These numbers do not include the multijet trigger simulation which reduces them by $\approx 60 \%$. The first error represents the statistical uncertainty. The second error comes from the theoretical uncertainty in the top cross section.

break each triplet into three dijet pairs with mass $M_{12}, M_{13}, M_{23}$ where the number refers to the jet in the triplet. We create normalized Dalitz variables:

$$
M_{i j}=\frac{M_{i j}^{2}}{M_{i j k}^{2}+M_{i}^{2}+M_{j}^{2}+M_{k}^{2}},
$$

with the condition $\mathrm{i} \neq \mathrm{j}$. We order them by size and obtain high, mid, and low Dalitz variables which sum up to 1 . The idea is to plot the different variables against each other and overlay the three plots at the end. All kinematic features of the decay are stored in these variables. To investigate the difference between signal region and background region we divide the mass plot in an "inside" (153 GeV/c $<M_{j j j}<189$ $\left.\mathrm{GeV} / c^{2}\right)$ and "outside" $\left(M_{j j j}<153 \mathrm{GeV} / c^{2}\right)$. Figure A.1 shows the three possible combinations of plotting the Dalitz variables against each other inside the top mass window for the PYTHIA $t \bar{t}$ sample. Overlaying the three plots from Figure A.1 leads to Figure A.2 which shows a comparison of $t \bar{t}$ Monte Carlo inside and outside the mass window. In Figure A.2 we can see the difference between the signal region and combinatoric background. The vertical branch on the top left plot is due to the W resonance in the top quark decay because of $\frac{M_{W}^{2}}{M_{t}^{2}} \approx 0.2$. The region of uncorrelated triplets (combinatorics) shows that the Dalitz variables are also uncorrelated. Figure 
A.3 shows the Dalitz plots for data. Due to the lack of statistics inside the mass window it is difficult to identify the events as top. However, in the right plot (outside the mass window) in Figure A.3 we see features that we expect from QCD events. QCD events often have a dijet structure where two hard jets balance each other out and are accompanied by softer jets. This leads to a population along the off-diagonal line in the Dalitz plot which means that out of the three Dalitz variables high $\approx$ mid. High and mid are both larger than low (which will be most likely the two soft jets) therefore we also expect a population along the y axis. Figure A.4 shows the three Dalitz variables from $t \bar{t}$ Monte Carlo and data separately in a one dimensional histogram. By demanding that Dalitz_low $>0.1$ we are selecting mainly real $t \bar{t}$ events and cut out most of the background. However, to see how the effect of this selection we point to Reference [42] where we use this study on an extended dataset.

\section{A.3 Identifying the b quark: b-tagging}

Most top quark analysis rely on the successful identification of the b quark in the $t \bar{t}$ decay. A technique called b-tagging is used to find the jets that originate from a bottom quark. This is possible due to the special properties of the b quark which forms $\mathrm{b}$ hadrons during the hadronization process. With a lifetime of roughly $c \tau=450 \mu \mathrm{m}$ these $\mathrm{b}$ hadrons are able to travel several centimeters inside the silicon tracker before they decay. This is called a secondary vertex and is used to identify the bottom jet [44].

\section{A.3.1 W boson}

If we consider the all-hadronic top decay we know that the $\mathrm{W}$ decays into two jets. The above mentioned b-tagging procedure can be used to identify the jet that most likely does not come from the $\mathrm{W}$. The invariant mass of the other two jets can now be associated with the W. Figure A.5 shows this anti b-tagged dijet mass as a Monte Carlo study done with a $t \bar{t}$ sample. However, this technique relies heavily on a good b-tag efficiency and a low fake rate. 

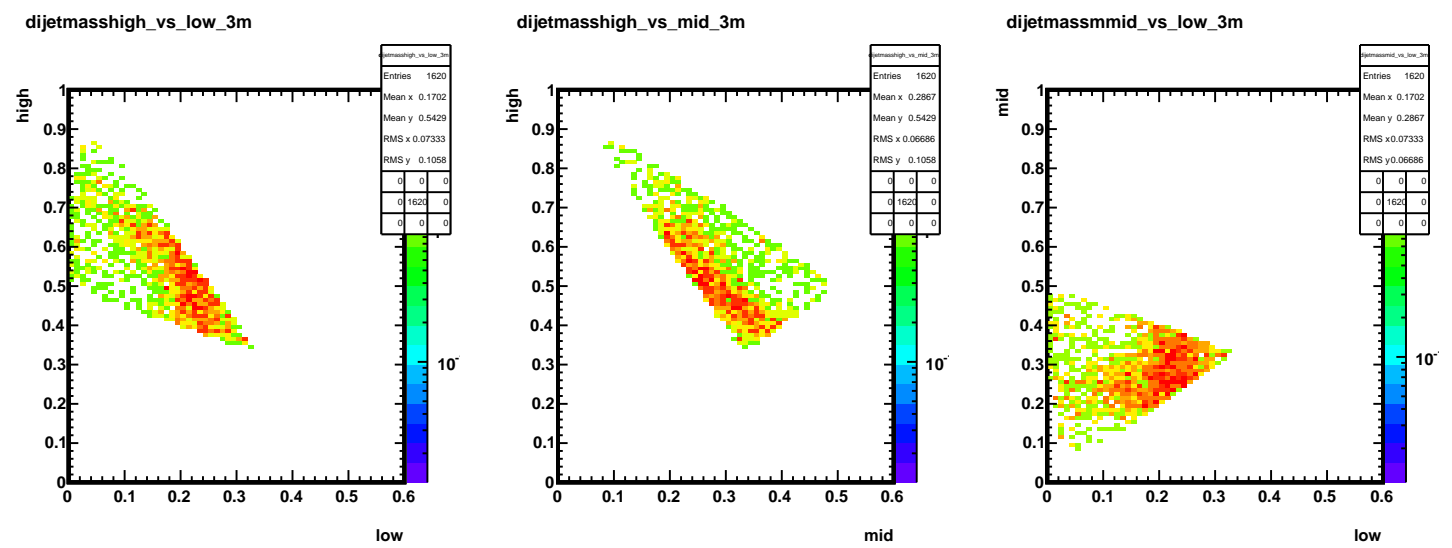

Figure A.1: The three combination of Dalitz variables in $t \bar{t}$ Monte Carlo inside the mass window. Left: high vs low, Middle: high vs mid, Right: mid vs low
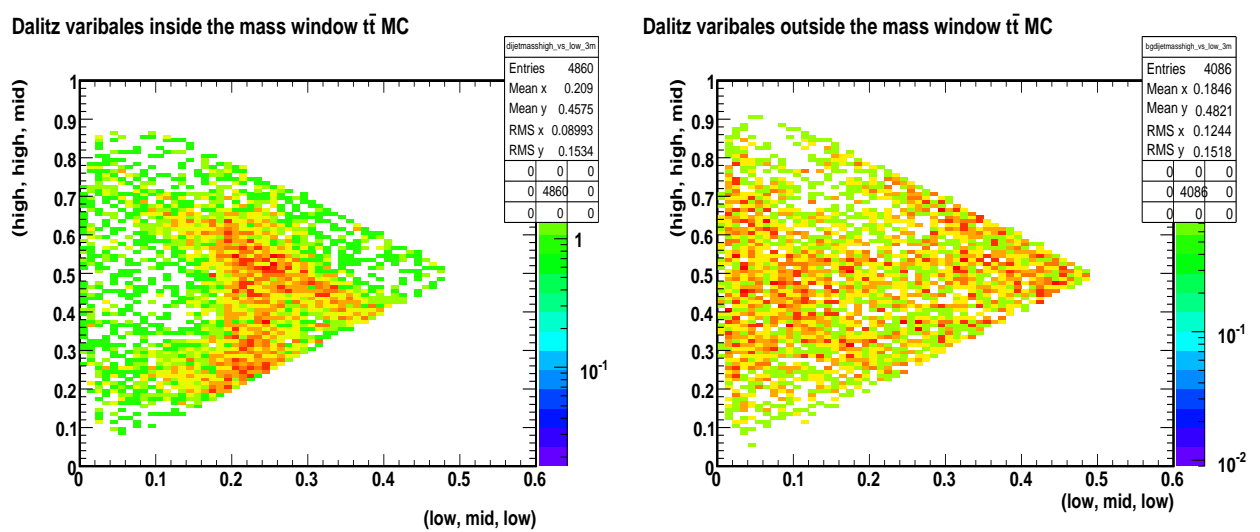

Figure A.2: Dalitz plots, all three combinations of variables overlaid for $t \bar{t}$ Monte Carlo. The vertical branch on the top left plot is due to the $\mathrm{W}$ resonance in the top quark decay because of $\frac{M_{W}^{2}}{M_{t}^{2}} \approx 0.2$. Left: inside the top mass window, Right: outside the top mass window.
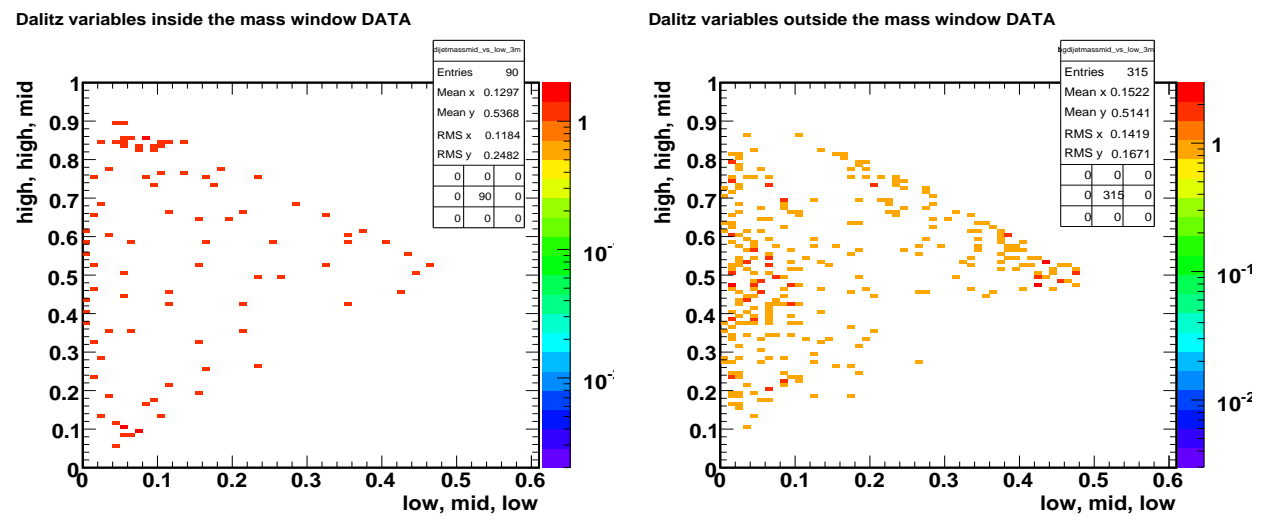

Figure A.3: Dalitz plots, all three combinations of variables overlaid for data. With only 30 events inside the mass window it is difficult to identify the events as either background or real top events. Left: inside the top mass window, Right: outside the top mass window. 

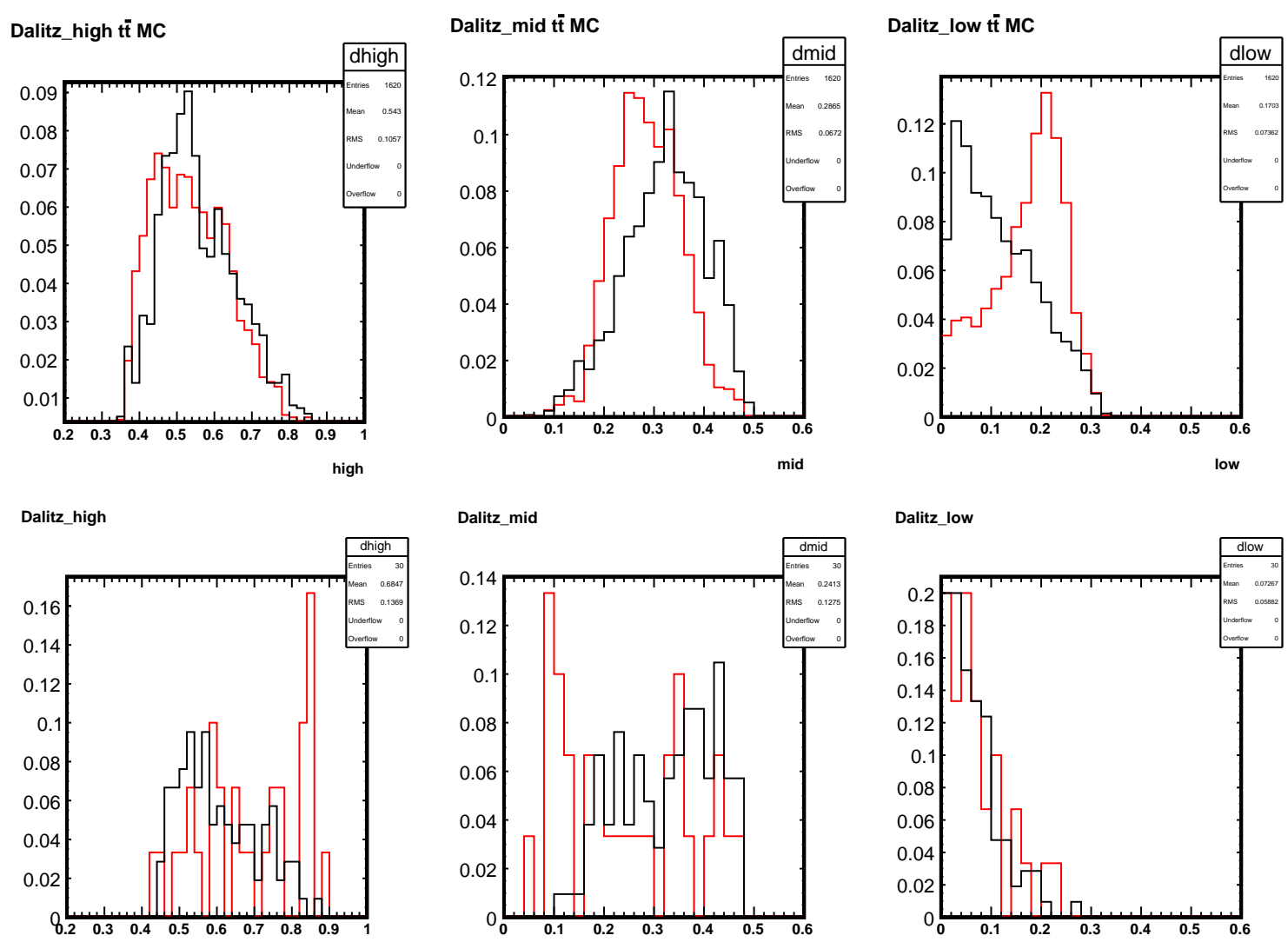

Figure A.4: Dalitz variables Top: $t \bar{t}$ Monte Carlo, Bottom: data. The histograms are normalized and show inside (red) and outside (black) the top mass window.

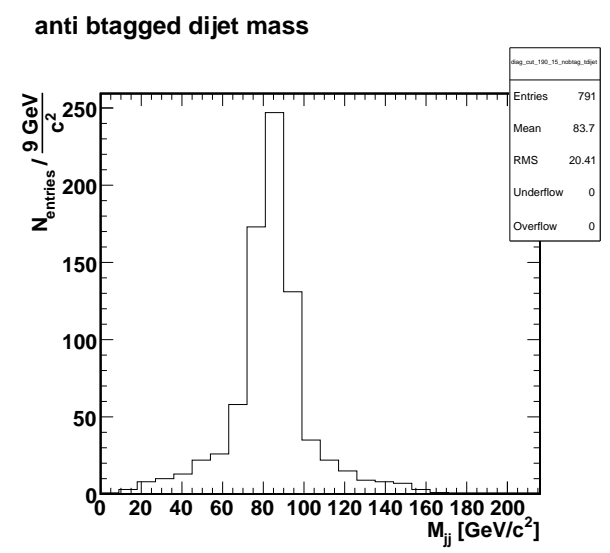

Figure A.5: We take the two non b-tagged jets in a triplet inside the top mass window and calculated the invariant mass for these two jets. We can see the $\mathrm{W}$ boson around $80 \mathrm{GeV} / c^{2}$ as expected. 


\section{A.4 Semi-leptonic decay}

Besides the all-hadronic top decay it is also possible to use the presented ensemble method for the semi-leptonic decay. There a different trigger path is necessary which chooses an isolated lepton, where isolated means that it has to be in a certain distance to a jet. The event also need a fairly large amount of missing $E_{T}$ as well as four jets. The requirement of the isolated lepton reduces the QCD background. A more detailed study of this decay channel can be found in Reference [42]. We find that we can loosen the diagonal cut requirement for this channel and therefore access a different kinematic region. We clearly see a signal around the top mass and Monte Carlo prediction agrees within statistical uncertainties with the number of observed top events.

\section{A.5 Conclusion}

We find that the jet ensemble technique works in the semi-leptonic channel. Further analysis of the all-hadronic channel give rise to the conclusion that the $2 \sigma$ excess around the top mass seems to be top like. 


\section{References}

[1] Francis Halzen, Allen Martin, Quarks and Leptons. John Wiley and Sons, 1984.

[2] Wikipedia, "Particles in the Standard Model." http://en.wikipedia.org/wiki/ File:Standard_Model_of_Elementary_Particles.svg.

[3] I. Aitchison, A. Hey, Gauge Theory in Particle Physics, Volume I and II. Taylor and Francis, 2003.

[4] W. Wagner, "Top quark physics in hadron collisions," Rep. Prog. Phys, vol. 68, pp. 2409-2494, 2005. hep-ph/0507207v2.

[5] P. Teixeira-Dias, "Higgs boson searches at LEP," J. Phys. Conf.Ser., vol. 110, 2008 .

[6] CDF and D $\phi$ Collaboration, "Combined CDF and D0 Upper Limits on Standard Model Higgs-Boson Production with up to $6.7 \mathrm{fb}^{-1}$ of Data," 2010. http:// arxiv.org/abs/1007.4587v1.

[7] Marion Lambacher, Study of fully hadronic ttbar decays and their separation from QCD multijet background events in the first year of the ATLAS experiment. $\mathrm{PhD}$ thesis, Ludwig-Maximilians-Universitaet Muenchen, Muenchen, Bayern, Deutschland, July 2007.

[8] Donald Perkins, Introduction to High Energy physics. Cambridge University press, 2001.

[9] Ellis, Stirling, Webber, QCD and Collider Physics. Cambridge University press.

[10] B.R. Webber, "Fragmentation and Hadronization," Int. J. Mod. Phys., pp. 577$606,2000$. 
[11] Eva Halkiadakis, "Proceedings for TASI 2009 Summer School on "Physics of the Large and the Small": Introduction to the LHC experiments," 2010. http:// arxiv.org/abs/1004.5564v1.

[12] S.P. Martin, "A supersymmetry primer," 2008. http://arxiv.org/abs/hep-ph/ 9709356v5.

[13] I. Aitchison, Supersymmetry in Particle Physics, Volume I. Cambridge University press, 2007.

[14] Herbi Dreiner, Peter Richardson, Michael H. Seymour, "Parton-Shower Simulations of R-parity Violating Supersymmetric Models," JHEP, 2000. http: //arxiv.org/abs/hep-ph/9912407v3.

[15] A. Quadt, "Top quark physics at hadron colliders," The European Physical Journal C-Particles and Fields, vol. 48, no. 3, pp. 835-1000, 2006.

[16] Fermilab, "Tevatron accelarator complex." http://www.fnal.gov/pub/today/ images08/00-0635D_hr.jpg

[17] Fermilab, "The Cockcroft-Walton." http://www.fnal.gov/pub/news04/update_ archive/update_9-10.html.

[18] R. Moore, "Tevatron Run II Series at the Enrico Fermi Laboratory (USA)," Atomic Energy, vol. 93, no. 6, pp. 930-933, 2002.

[19] Sourabh Dube, Search for Supersymmetry at the Tevatron using the Trilepton signature. PhD thesis, Rutgers, The State University of New Jersey, New Brunswick, NJ, USA, October 2008.

[20] "Accelerator Concepts." http://www-bdnew.fnal.gov/operations/rookie_ books/Concepts_v3.6.pdf.

[21] CDF Collaboration, "The CDF detector: an overview," Nuclear Instruments and Methods in Physics Research A, vol. 271, no. 3, pp. 387-403, 1988. 
[22] Fermilab, "Elevation view of CDF." http://hep-www.px.tsukuba.ac.jp/ hara/ isl/cdfii-elev.GIF.

[23] Fermilab, "Silicon tracking system." http://www-cdf.fnal.gov/upgrades/ silicon/img6.gif.

[24] A. Abulencia, et al., "Measurements of inclusive $\mathrm{W}$ and $\mathrm{Z}$ cross sections in ppar collisions at $1.96 \mathrm{TeV}, "$ J. Phys. G: Nucl. Part. Phys., vol. 34, p. 2457, 2007.

[25] K. Nakamura et al., "Particle Data Group," J. Phys. G, vol. 37, no. 075021, 2010.

[26] V. Sorin, "CDF Trigger System." dept.physics.upenn.edu/ thomsone/ hepseminars/talks/2006_sorin.pdf.

[27] A. Mitra et al., "Study of Multijet Triggers for all-hadronic Higgs search," CDF/ANAL/TRIGGER/CDFR/9954, 2005.

[28] T. Sjöstrand et al., "PYTHIA," Comp. Phys. Comm., vol. 135, p. 238, 2001.

[29] "GEANT," Nuclear Instruments and Methods in Physics Research A, vol. 506, no. 3, pp. 250-330, 2003.

[30] M.L. Mangano, M. Moretti, F. Piccinini, R. Pittau, A. Polosa, "AlPGEN," J. High. Energy. Phys., vol. 0307, p. 001, 2003.

[31] S. Frixione and B. Webber, "MC@NLO," J. High. Energy. Phys., vol. 0206, p. 029, 2002.

[32] Rouven Essig, PHYSICS BEYOND THE STANDARD MODEL: SUPERSYMMETRY, DARK MATTER, AND LHC PHENOMENOLOGY. PhD thesis, Rutgers, The State University of New Jersey, New Brunswick, NJ, USA, October 2008.

[33] E. Halkiadakis, A. Lath, C. Seitz, D. Hare, G. Jain, T. Lou, "Internal note: Search for a New Hadronic Resonance using Jet Ensembles with CDF," CDF/PHYS/EXOTIC/CDFR/10192. 
[34] E. Halkiadakis, A. Lath, C. Seitz, D. Hare, G. Jain, T. Lou, "Public note: Search for a New Hadronic Resonance using Jet Ensembles with CDF," CDF/DOC/EXOTIC/PUBLIC/10256.

[35] E. Halkiadakis, A. Lath, C. Seitz, D. Hare, G. Jain, T. Lou, "Public website: Search for a New Hadronic Resonance using Jet Ensembles with CDF," http: //www-cdf.fnal.gov/physics/exotic/r2a/20100715.multijetsensembles/.

[36] Gerald C. Blazey et al., "Run II Jet Physics," arXiv:hep-ex/0005012v2

[37] Jay R. Dittmann, "CDF RUN 2 Jet Algorithms." http://www.pa.msu.edu/ huston/tev4lhc/tev4lhc-dittmann-cdfjets-20041201.pdf.

[38] A. Bhatti et al., "Determination of the Jet Energy Scale at the Collider Detector at Fermilab," Nuclear Instruments and Methods in Physics Research A, vol. 566, no. 2, pp. 375-412, 2006. arXiv:hep-ex/0510047v1

[39] CDF Collaboration, C. Hays, P. Tamburello, A. Kotwal, P. Wittich, and R. Snider, "COT Pattern Recognition Algorithm and Oline Code," CDF/DOC/TRACKING/CDFR/6992, 2004.

[40] Carsten Rott, Daniela Bortoletto, "Primary Vertex Algorithm Comparison for high $p_{T}$ Physics," CDF/DOC/MISSING ET/CDFR/6472, 2003.

[41] D. Acosta et. al., "The performance of the CDF luminosity monitor," Nuclear Instruments and Methods in Physics Research Section A: Accelerators, Spectrometers, Detectors and Associated Equipment, vol. 494, no. 1-3, pp. 57-62, 2002.

[42] E. Halkiadakis, A. Lath, C. Seitz, D. Hare, G. Jain, T. Lou, "Jet ensemble studies using semi-leptonic and all-hadronic $t \bar{t}$ decays," CDF/DOC/EXOTIC/CDFR/10346.

[43] R. H. Dalitz, "Decay of $\tau$ Mesons of Known Charge," Phys. Rev., vol. 94, no. 4, pp. 1046-1051, 1954.

[44] T. Wright, for the CDF and D $\phi$ Collaborations, "B-Tagging at CDF and D $\phi$, Lessons for LHC," http://arxiv.org/abs/0707.1712. 